\title{
Mass Spectrometric Approaches to Characterization of Chemical Species Containing Metallic lons
}

\author{
Guodong Gu \\ West Virginia University
}

Follow this and additional works at: https://researchrepository.wvu.edu/etd

\section{Recommended Citation}

Gu, Guodong, "Mass Spectrometric Approaches to Characterization of Chemical Species Containing Metallic Ions" (2012). Graduate Theses, Dissertations, and Problem Reports. 3583.

https://researchrepository.wvu.edu/etd/3583

This Dissertation is protected by copyright and/or related rights. It has been brought to you by the The Research Repository @ WVU with permission from the rights-holder(s). You are free to use this Dissertation in any way that is permitted by the copyright and related rights legislation that applies to your use. For other uses you must obtain permission from the rights-holder(s) directly, unless additional rights are indicated by a Creative Commons license in the record and/ or on the work itself. This Dissertation has been accepted for inclusion in WVU Graduate Theses, Dissertations, and Problem Reports collection by an authorized administrator of The Research Repository @ WVU.

For more information, please contact researchrepository@mail.wvu.edu. 


\title{
Mass Spectrometric Approaches to Characterization of Chemical Species Containing Metallic Ions
}

\author{
Guodong Gu
}

Dissertation submitted to the Eberly College of Arts and Sciences at West Virginia University

in partial fulfillment of the requirements for the degree of

\author{
Doctoral of Philosophy \\ in \\ Chemistry
}

Fred L. King, Ph. D., Chair

Ronald B. Smart, Ph. D.

Björn Söderberg, Ph. D.

Jonathan Boyd, Ph. D.

Patrick S. Callery, Ph. D.

C. Eugene Bennett Department of Chemistry

Morgantown, West Virginia

2012

Keywords: Mass spectrometry, Glow Discharge, Metal, Internal Energy Distribution, Peptide and Amino Acid

Copyright 2012 Guodong Gu 


\title{
ABSTRACT \\ Mass Spectrometric Approaches to Characterization of Chemical Species Containing Metallic Ions
}

\author{
Guodong Gu
}

Mass spectrometry is a widely used analytical technique for both qualitative and quantitative analysis of physical, chemical, and biological compounds by measuring mass to charge ratio $(\mathrm{m} / \mathrm{z})$ of particles. It can supply elemental composition, molecular weight, and structure information of chemical compounds. The high sensitivity and analysis speed enable it to be an outstanding tool for chemical analysis. A Quadrupole Ion Trap Mass Spectrometry (QITMS) and a pulsed Glow Discharge Time-of-Flight Mass Spectrometry (GD-TOFMS) are employed in this study.

Internal energy distribution, $\mathrm{P}(\mathrm{E})$, of collision induced dissociation in QITMS is studied by using "thermometer molecules", whose fragmentation pathway consists of several consecutive reactions with known activation energies. A plot of $\mathrm{P}(\mathrm{E})$ versus internal energy indicates the amount of internal energy deposited to precursor ions and proportion of ions with different energy. The experiment shows that there is a limit of deposited energy to the precursor ions and multiple collision induced 
dissociation contributes minimally.

Although Glow Discharge Mass Spectrometry (GDMS) is best known for its utility in trace element analysis, recent work demonstrates that the technique can also provide chemical speciation information. By carefully tuning parameters such as sampling distance, temporal regime, discharge gas pressure, pulse frequency, and duty cycle, a specific desired plasma environment can be produced to generate ions used for speciation. For the iron oxides, species specific variations in ratios between $\mathrm{Fe}^{+}$and $\mathrm{FeOH}^{+}$signal intensities provide the ability to discriminate between $\mathrm{FeO}, \mathrm{Fe}_{2} \mathrm{O}_{3}$, and $\mathrm{Fe}_{3} \mathrm{O}_{4}$. Iron-oxygen cluster generation correlates with oxygen abundance in the original sample as seen in these comparisons. Three groups of metal oxides including Cobalt oxides $\left(\mathrm{CoO}\right.$ and $\left.\mathrm{Co}_{3} \mathrm{O}_{4}\right)$, niobium oxides $\left(\mathrm{NbO}, \mathrm{NbO}_{2}\right.$, and $\left.\mathrm{Nb}_{2} \mathrm{O}_{5}\right)$, and copper oxides $\left(\mathrm{Cu}_{2} \mathrm{O}\right.$, and $\left.\mathrm{CuO}\right)$ are tested with GDMS following iron oxides experiment.

The direct analysis of bio-samples, such as amino acids and peptide, by GDMS is also explored. Three different sample preparation methods are employed to explore the role of solvents or additives. Glycerol is explored to mimic Fast Atom Bombardment (FAB) and Liquid Secondary Ion Mass Spectrometry (LSIMS) experimental condition in the first method, but no molecular ion or characteristic ions are detected. In the second method, samples are dissolved in deionized water and a sample solution droplet was deposited on the sample disk. A characteristic ion, m/z 76, from cysteine detected arising from carboxylic acid loss. In the last method, 
bio-samples were dissolved in $10 \mathrm{mM}$ cesium chloride solution instead of deionized water, but the expected cesium related cluster ion was not detected in the spectra. Except for the 76 peak from cysteine, there are no molecular or characteristic ions detected. 


\section{DEDICATED TO}

My parents, wife, and family 


\section{ACKNOWLEDGEMENTS}

First and foremost, I would like to express my paramount gratitude to my advisor and mentor, Dr. Fred L. King, for his valuable guidance, comments, and encouragement throughout my graduate study. Not only was I taught extensive knowledge and skills by him, but also methods to think and solve issues independently.

I am grateful to my committee members Dr. Ronald Smart, Dr. Björn Söderberg, Dr. Jonathan Boyd, and Dr. Patrick S. Callery for spending time on my graduate study and dissertation. Their helpful suggestion and valuable discussion are greatly acknowledged.

I would like to thank the entire members of the King Group whom I have had pleasure to work with: Dr. Na Zhang, Dr. Jennifer N. Robertson-Honecker, Dr. Ting Zhao, Megan DeJesus, Teerapat Rojsajjakul, Han Wang, Yan pan, Xiaqing Xu, Alex Pavkovich, and Donald Bennett, for their friendship and assistance. I would also express my gratitude to Dr. Yuchen Lu for his valuable discussion on my research.

Financial supports from the C. Eugene Bennett Chemistry Department and the Eberly College of Arts and Sciences at WVU as well as the U.S. Department of Energy are greatly appreciated. Technical assistance of Don Feathers, Allen Burns, Randall Eaglen, Phillip Tucker, and J.R. Taylor is gratefully acknowledged too. 
Finally, I would like to thank my parents, Chuantai Gu and Shuyuan Shen, and my wife, Changchang Xiao, for giving me endless of love, encouragement, and support over my life. Without them, I would not be where I am today. 


\section{Table of Contents}

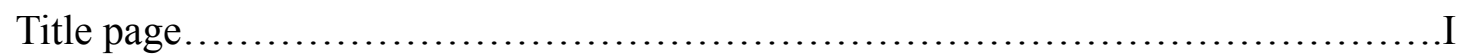

Abstract.....................................................................

Acknowledgement...................................................... VI

Table of contents............................................................... VII

List of figures.........................................................

List of tables.........................................................

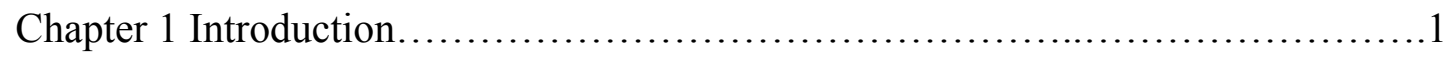

1.1 Metals and Chemical Species Containing Metallic Ions.......................

1.2 Glow Discharge Time-of-Flight Mass spectrometry .........................

1.2.1 Glow Discharge Processes........................................ 3

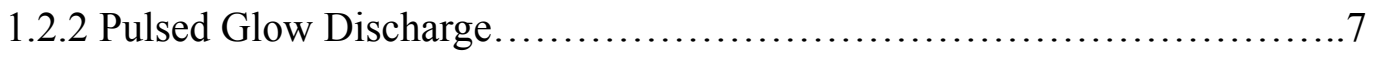

1.2.3 Pulsed Glow Discharge Time-of-Flight Mass Spectrometer

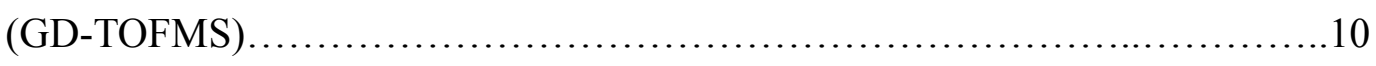

1.3 Electrospray - Ion Trap Mass Spectrometry ............................. 13

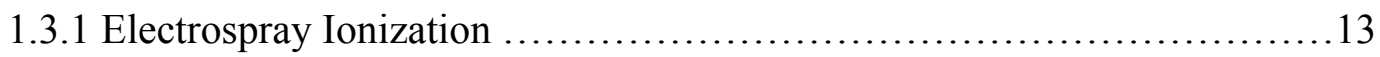

1.3.2 Quadrupole Ion Trap Mass Analyzer.................................17

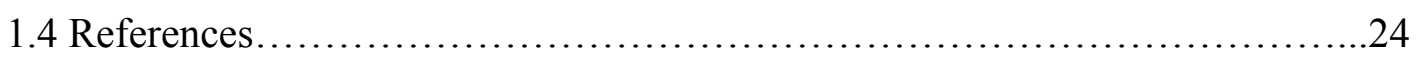


Chapter 2 Internal Energy Distribution of Collision Induced Dissociation in Quadrupole Ion Trap Mass Spectrometry......................................27

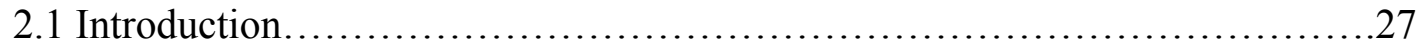

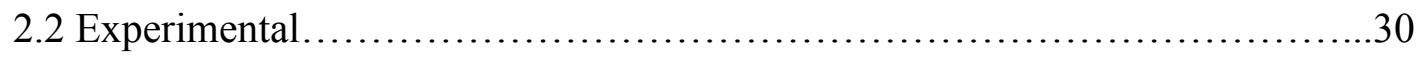

2.3 Result and Discussion................................................ 31

2.4 Conclusion..............................................................

2.5 References...................................................... 37

Chapter 3 Direct Iron Oxide $\left(\mathrm{Fe}_{\mathrm{x}} \mathrm{O}_{\mathrm{y}}\right)$ Speciation in Solid State Materials by Pulsed Millisecond Radio Frequency Glow Discharge Time-of-Flight Mass

Spectrometry ..................................................... 40

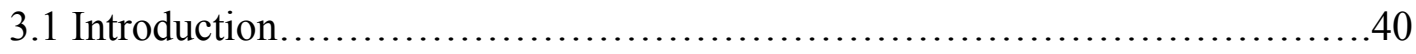

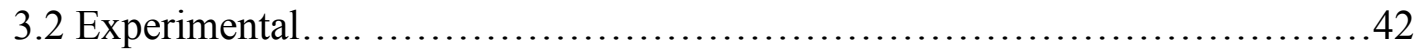

3.3 Results and Discussion..........................................44

3.3.1 Optimization of Temporal Regimes................................44

3.3.2 Optimization of Sampling Distance..............................49

3.3.3 Optimization of Glow Discharge Operating Parameters ...............55

3.3.3.1 Operating Power...........................................55

3.3.3.2 Discharge Gas Pressure.....................................56

3.3.3.3 Power Pulse Width and Duty Cycle.............................58

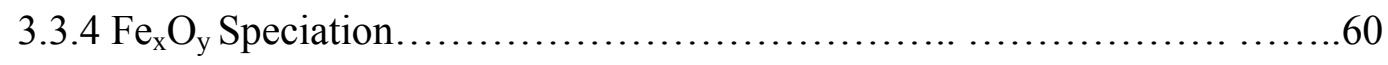


Chapter 4 Extending the Application of Pulsed Millisecond Radio Frequency Glow

Discharge Time-of-Flight Mass Spectrometry to the Characterization of Other

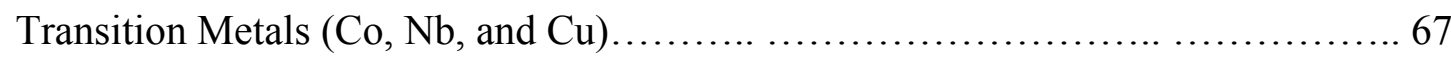

4.1 Introduction........................................................67

4.2 Experimental...................................................6 68

4.3 Result and Discussion............................................. 70

4.3.1 Optimization of Temporal Regimes............................. 70

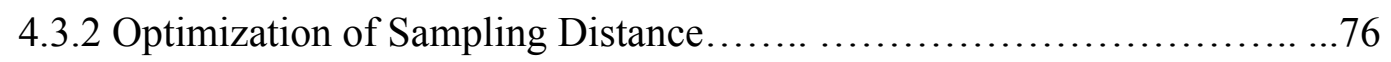

4.3.3 Optimization of Operating Power..................................77

4.3.4 Transition Metal Oxides Analysis....................................... 80

4.3.4.1 Cobalt Oxides Analysis.......................................80

4.3.4.2 Niobium Oxides Analysis......................................80

4.3.4.3 Copper Oxides Analysis.....................................81

4.4 Conclusion....................................................... 85

4.5 References........................................................... 86

Chapter 5 A Preliminary Exploration of Peptide and Amino Acid Identification by Pulsed Glow Discharge Mass Spectrometry................................ 88

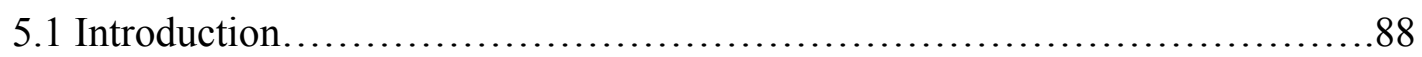


5.3 Result and Discussion................................................ 93

5.3.1 Sample and Glycerol Mixture Analysis...............................93

5.3.2 Sample Solution Analysis........................................ 96

5.3.3 Sample and $\mathrm{CsCl}$ Solution Analysis..................................100

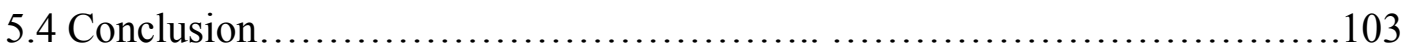

5.5 References....................................................... 105 


\section{Table of Figures}

Figure 1.1 Periodic table of the elements....................................

Figure 1.2 Schematic structure of a glow discharge source........................4

Figure 1.3 Structure of Glow Discharge Time-of-Flight mass spectrometer..........10

Figure 1.4 Schematic of electrospray ionization process........................15

Figure 1.5 Schematic view of quadrupole ion trap. The term $r_{0}$ and $Z_{0}$ are the distance from center to ring electrode and endcap electrode. 18

Figure 1.6 Mathieu stability diagram of quadrupole ion trap. Ions are stable in both $r$ and $\mathrm{z}$ direction if its $\mathrm{a}_{\mathrm{z}}$ and $\mathrm{q}_{\mathrm{z}}$ value are in the shaded area

Figure 2.1 Tandem mass spectra of the $\left(\mathrm{C}_{2} \mathrm{H}_{5} \mathrm{O}\right)_{3} \mathrm{PO} \cdot \mathrm{H}^{+}$ion at (a) $8 \% \mathrm{CID}$, (b) $10 \%$ CID, (c) $20 \% \mathrm{CID}$, (d) $30 \% \mathrm{CID}$, (e) $40 \%$ CID in a quadrupole ion trap mass spectrometry. .34

Figure 2.2 $\mathrm{P}(\mathrm{E})$ of the $\left(\mathrm{C}_{2} \mathrm{H}_{5} \mathrm{O}\right)_{3} \mathrm{PO} \cdot \mathrm{H}^{+}$ion activated by $\mathrm{CID}$ in a quadrupole ion trap mass spectrometry. Each data point is the result of 3 measurements and error bars represent one standard deviation. .35

Figure 3.1 Data acquisition of RF GD-TOFMS .45

Figure 3.2 Time resolved mass spectra of (a) pure $\mathrm{Ag}$ and (b) $25 \% \mathrm{FeO} / \mathrm{Ag}$ samples during the prepeak ( $0.3 \mathrm{~ms}$ after power-on). Discharge pressure 0.4 Torr, operating power $80 \mathrm{~W}$, pulse duration $5 \mathrm{~ms}$ with $25 \%$ duty cycle and sampling distance 10 $\mathrm{mm}$.

Figure 3.3 Time resolved mass spectra of (a) pure $\mathrm{Ag}$ and (b) $25 \% \mathrm{FeO} / \mathrm{Ag}$, (c) $25 \%$ $\mathrm{Fe}_{2} \mathrm{O}_{3} / \mathrm{Ag}$, and (d) $25 \% \mathrm{Fe}_{3} \mathrm{O}_{4} / \mathrm{Ag}$ during the plateau (1.3 $\mathrm{ms}$ after power-on). Discharge pressure 0.4 Torr, operating power $80 \mathrm{~W}$, pulse duration $5 \mathrm{~ms}$ with $25 \%$ 
Figure 3.4 Time resolved mass spectra of (a) pure $\mathrm{Ag}$ (b) $25 \% \mathrm{FeO} / \mathrm{Ag}$, (c) $25 \%$ $\mathrm{Fe}_{2} \mathrm{O}_{3} / \mathrm{Ag}$, and (d) $25 \% \quad \mathrm{Fe}_{3} \mathrm{O}_{4} / \mathrm{Ag}$ samples during the afterpeak (5.3 ms after power-on, $1.3 \mathrm{~ms}$ after power-off). Discharge pressure 0.4 Torr, operating power $80 \mathrm{~W}$, pulse duration $5 \mathrm{~ms}$ with $25 \%$ duty cycle and sampling distance $10 \mathrm{~mm}$.

Figure 3.5 Spatial effect on ion signals for representative $\mathrm{Fe}_{3} \mathrm{O}_{4}: \mathrm{Ag}=25: 75(\mathrm{~m}: \mathrm{m})$ sample pellet. The glow discharge power is $80 \mathrm{w}$, and the discharge gas pressure is 0.4 torr. Each data point is the result of 3 measurements and error bars represent one standard deviation from the mean value.

Figure 3.6 Power effect on ion signals for $\mathrm{Fe}_{3} \mathrm{O}_{4}: \mathrm{Ag}=25: 75$ (m:m) sample pellet. The glow discharge pressure is 0.4 torr Ar and the sampling distance is $10 \mathrm{~mm}$. Each data point is the result of 3 measurements and error bars represent one standard deviation from the mean value. .56

Figure 3.7 Pressure effect on ion signals for $\mathrm{Fe}_{3} \mathrm{O}_{4}: \mathrm{Ag}=25: 75$ (m:m) sample pellet. The glow discharge power is $80 \mathrm{~W}$ and the sampling distance is $10 \mathrm{~mm}$. Each data point is the result of 3 measurements and error bars represent one standard deviation from the mean value.

Figure 3.8 Pulse width effect on ion signals at $25 \%$ duty cycle for $\mathrm{Fe}_{3} \mathrm{O}_{4}: \mathrm{Ag}=25: 75$ (m:m) sample pellet. The glow discharge power is $80 \mathrm{~W}$, the discharge gas pressure is 0.4 torr Ar, and the sampling distance is $10 \mathrm{~mm}$. Each data point is the result of 3 measurements and error bars represent one standard deviation from the mean value. .59

Figure 3.9 Duty cycle effect studied with $\mathrm{Fe}_{3} \mathrm{O}_{4}: \mathrm{Ag}=25: 75$ (m:m) pellet. The glow discharge power is $80 \mathrm{~W}$, the discharge gas pressure is 0.4 torr Ar, and the sampling distance is $10 \mathrm{~mm}$. Each data point is the result of 3 measurements and error bars represent one standard deviation from the mean value. 
Figure 3.10 $\mathrm{Fe}$ and $\mathrm{FeO}$ related peak intensity ratios for three iron oxides. Glow discharge operating power $80 \mathrm{~W}$ with $2 \mathrm{~ms}$ pulse width and $10 \%$ duty cycle, gas pressure 0.3 torr, sampling distance $15 \mathrm{~mm}$. Each data point is the result of 6 measurements and error bars represent one standard deviation from the mean value.

Figure 4.1 Time resolved mass spectra of (a) pure Ag, (b) $25 \% \mathrm{CoO} / \mathrm{Ag}$, and (c) $25 \%$ $\mathrm{Co}_{3} \mathrm{O}_{4} / \mathrm{Ag}$ during the plateau (2.0 ms after power-on). Discharge pressure 0.3 Torr, peak power $80 \mathrm{~W}$, frequency $50 \mathrm{~Hz}$, duty cycle $10 \%$ duty cycle and sampling distance $10 \mathrm{~mm}$.

Figure 4.2 Time resolved mass spectra of (a) pure $\mathrm{Ag}$, (b) $25 \% \mathrm{CoO} / \mathrm{Ag}$, and (c) $25 \%$ $\mathrm{Co}_{3} \mathrm{O}_{4} / \mathrm{Ag}$ samples during the afterpeak (0.2 ms after power-off). Discharge pressure 0.3 Torr, peak power $80 \mathrm{~W}$, frequency $50 \mathrm{~Hz}$, duty cycle $10 \%$ duty cycle and sampling distance $10 \mathrm{~mm}$ .76

Figure 4.3 Spatial effect on ion signals for $25 \% \mathrm{CoO} / \mathrm{Ag}$ pellet. Data is colleted in afterpeak period at $0.2 \mathrm{~ms}$ after power-off. The discharge gas pressure is 0.3 Torr; peak power is $80 \mathrm{~W}$ with $50 \mathrm{~Hz}$ frequency and $10 \%$ duty cycle. Each data point is the result of 3 measurements and error bars represent one standard deviation from the mean value. 78

Figure 4.4 Power effect on ion signals for $25 \% \mathrm{Co}_{3} \mathrm{O}_{4} / \mathrm{Ag}$ sample pellet. Data are colleted in afterpeak period at $0.2 \mathrm{~ms}$ after power-off. The discharge gas pressure is 0.3 Torr; the sampling distance is $10 \mathrm{~mm}$; pulse frequency is $50 \mathrm{~Hz}$ with $10 \%$ duty cycle. Each data point is the result of 3 measurements and error bars represent one standard deviation from the mean value.

Figure 4.5 Mass spectra of (a) $25 \% \mathrm{NbO} / \mathrm{Ag}$ (b) $25 \% \mathrm{NbO}_{2} / \mathrm{Ag}$, and $25 \% \mathrm{Nb}_{2} \mathrm{O}_{5} / \mathrm{Ag}$ pellets during the afterpeak $(5.2 \mathrm{~ms})$. Discharge pressure 0.3 Torr, operating power 80 $\mathrm{W}$, pulse duration $5 \mathrm{~ms}$ with $25 \%$ duty cycle and sampling distance $10 \mathrm{~mm}$. . .83 
Figure 4.6 Mass spectra of (a) $25 \% \mathrm{Cu}_{2} \mathrm{O}$ and $\mathrm{Ag}$ (b) $25 \% \mathrm{CuO}$ and $\mathrm{Ag}$ pellets during the afterpeak $(5.1 \mathrm{~ms})$. Discharge pressure 0.3 Torr, peak power $80 \mathrm{~W}$, frequency 50 $\mathrm{Hz}$, duty cycle $25 \%$ duty cycle and sampling distance $10 \mathrm{~mm}$ $\ldots 84$

Figure 5.1 Structure of twenty amino acids 92

Figure 5.2 Mass spectra of (a) Glycerol and (b) Glycerol + Gly samples during the afterpeak (0.2 ms after power-off). Discharge pressure 0.3 Torr, sampling distance 15 mm, peak power $80 \mathrm{~W}$ with $100 \mathrm{~Hz}$ frequency and $10 \%$ duty cycle. .95

Figure 5.3 Mass spectra of (a) Ag, (b) Leucine, (c) Cysteine, and (d) GlyGlyHis samples during the afterpeak (0.2 ms after power-off). Discharge pressure 0.3 Torr, sampling distance $15 \mathrm{~mm}$, peak power $80 \mathrm{~W}$ with $100 \mathrm{~Hz}$ frequency and $10 \%$ duty cycle. .99

Figure 5.4 Standard EI mass spectrum of cysteine 100

Figure 5.5 Mass spectra of (a) $\mathrm{CsCl}$, (b) $\mathrm{CsCl}+$ Cysteine, and (c) $\mathrm{CsCl}+$ GlyGlyHis samples during the afterpeak (0.2 ms after power-off). Discharge pressure 0.3 Torr, sampling distance $15 \mathrm{~mm}$, peak power $80 \mathrm{~W}$ with $100 \mathrm{~Hz}$ frequency and $10 \%$ duty cycle. 103 


\section{Table of Tables}

Table 2.1 Thermochemical data of triethyl phosphate.............................35

Table 3.1 Pulsed RF GD-TOFMS operating parameters...........................44

Table 4.1 Pulsed RF GD TOF-MS Operating Parameters.........................69

Table 5.1 Pulsed RF GD TOF-MS Operating Parameters.........................93 


\section{Chapter 1}

\section{Introduction}

\subsection{Metals and Chemical Species Containing Metallic Ions}

A metal is generally defined as a substance or mixture that is relatively shiny and malleable and is a good conductor of both electricity and heat. In reactions, metals tend to transfer electrons to nonmetals and form ionic compounds. ${ }^{1}$ Metals can also be identified based on their positions in periodic table (Figure 1.1), noting that nearly $80 \%$ of elements in periodic table are metals.

Periodic table of the elements

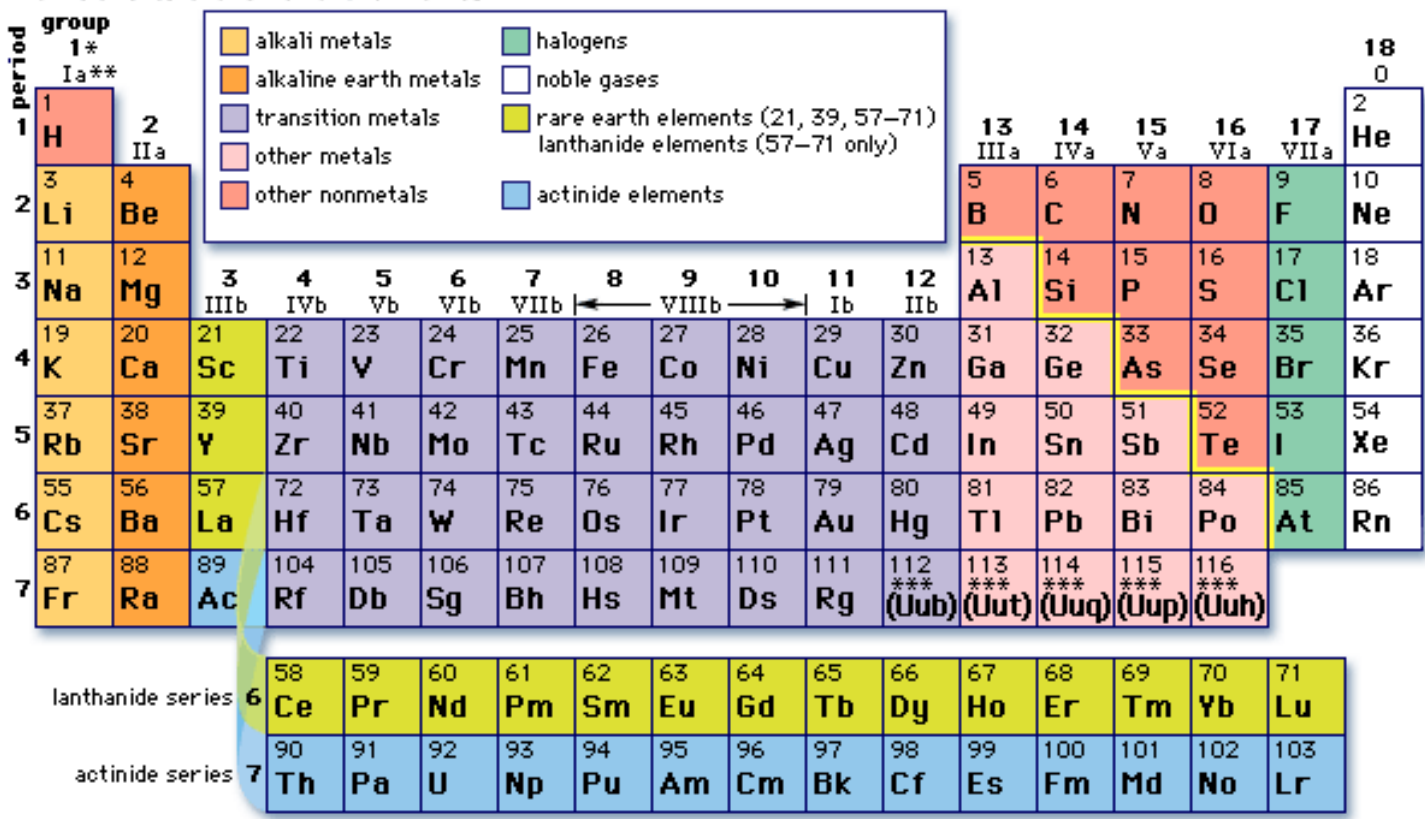

* Numbering system adopted by the International Union of Pure and Applied Chemistry (IUPAC).

** Numbering system widely used, especially in the U.S., from the mid-20th century.

*** Discoveries of elements 112-116 are claimed but not confirmed. Element names and symbols in parentheses are temporarily assigned by IUPAC.

Figure 1.1 Periodic table of the elements. 
Because the outer electrons of metal atoms are readily shared across metallic bonds, the resistance for electron movement and energy transfer is small; thus, electrical and thermal conductivity are high. Because of high electrical conductivity, metals are used in electrical appliances and carry an electric current over a distance with little energy lost. Copper, aluminum, and silver are the primary metals used in electrical distribution systems. The good thermal conductivity of metals is used in either conducting heat to specific target such as in cooking utensils or dissipating heat away from certain objects to avoid overheating such as cooling fins on circuit boards. In appearance, metals are usually shiny and lustrous. This is because visible light can not be transmitted through the bulk metal microstructure and the large population of mobile electrons on the surface will absorb and reflect the light. Some metals have high reflectivity and can be used to make mirrors and high precision instruments. Although metals usually have higher densities than nonmetals, the range of these densities is large. Lithium has the lowest density as $0.53 \mathrm{~g} / \mathrm{cm}^{3}$, about half of water density; osmium has the greatest density, $22.61 \mathrm{~g} / \mathrm{cm}^{3}$. The highly packed crystal lattice of the metallic structure contributes most to the high density of metals. Other factors, such as atomic radius, nuclear charge, etc., also impact density. ${ }^{2}$ Ductility is an important mechanical property for metals. It is based on inherent capacity of plastic deformation and the non directional nature of metallic bonding. Gold has the best ductility in metals. One gram of gold can be stretched to a wire 2.2 miles in length and $4.3 \mathrm{E} 10^{-6} \mathrm{~m}$ in diameter. Some metals with specific properties have found specialized uses. For example, radioactive metals like uranium (U) can be used as 
fuels in nuclear power plants to produce electric power.

Metals typically exist in the natural environment in both elemental and compound forms. The exceptions are metals like alkali and alkaline metals, which are extremely reactive, and metals like gold and platinum, which are extremely non-reactive. Metal atoms usually tend to lose electrons and get oxidized. Pure metals in the air react with oxygen and form metal oxides. A typical metal oxidation reaction is the rusting of iron. Not all metal oxidation processes harm the metal's physical properties like rusting does. Metals such as aluminum, magnesium, and titanium form an oxidation layer on the surface, which protects the bulk metal by stopping further oxidation. Besides the oxidation process of metals, which is a combination reaction, metals can also undergo displacement reactions. A good example is the reaction between a metal and acid. Because of high activity of metals, chemical species containing metallic ions are found in many matrices such as water, air, soil, and biological system.

\subsection{Glow Discharge Time-of-Flight Mass spectrometry}

\subsubsection{Glow Discharge Processes}

A glow discharge is initiated by the establishment of a potential difference between two electrodes in a low pressure environment. In its classic and simplest structure as shown in Figure 1.2, the glow discharge device is a cell at low pressure (0.1-10.0 Torr). The cell is typically filled with a noble gas, typically argon for an 
analytical glow discharge, but other gases can also be used. Two electrodes are placed in two sides of the cell. Samples can either work as, or be contained in, surface of cathode; anode material is not sampled. ${ }^{3,4}$ Principal samples analyzed in glow discharge are solid metal materials. The conductive samples can be sputtered and directly analyzed by direct current (DC) glow discharge, but non-conductive materials must be mixed with conductive matrix such as graphite to get a sustainable discharge. Otherwise, radio frequency (RF) glow discharge source could be used to analyze non-conducting materials directly. Other applications such as the examination of thin films and solution residues are also possible..$^{5-7}$

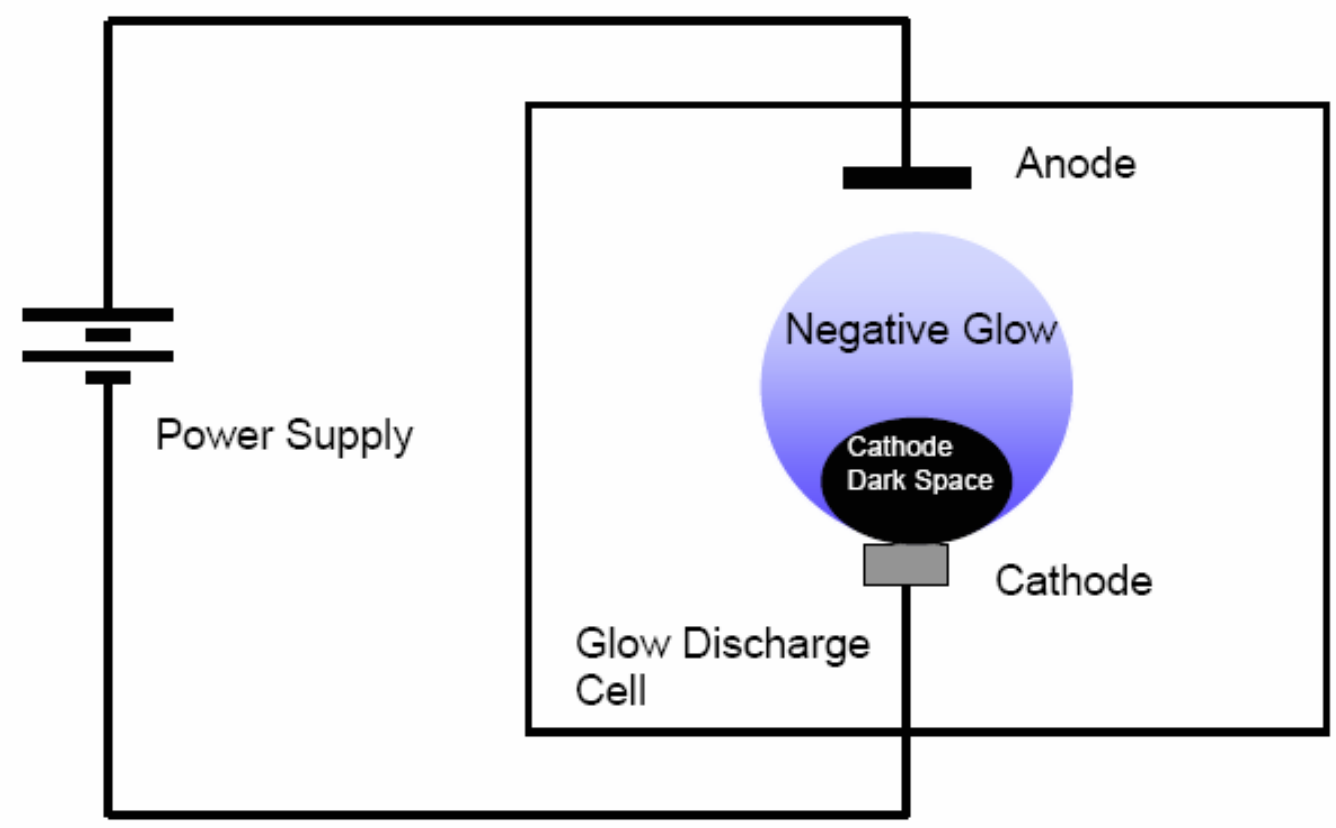

Figure 1.2 Schematic structure of a glow discharge source

When an electric potential of 250-2000 volts is applied between those electrodes, energy sufficient to break down the discharge gas electrically, positively charged $\mathrm{Ar}^{+}$ 
ions and free electrons are formed. Free electrons are accelerated towards the anode, while colliding with argon atoms in discharge gas on their way. The resulting electron ionization process will in turn generate more argon ions and new electrons as a result sustaining the discharge. Argon ions then move toward the cathode, impact its surface, and transfer kinetic energy to it. If species on the surface receive sufficient energy to overcome their lattice binding, they will be sputtered out mainly as neutral atoms. The sputtered neutral population, which can be excited or ionized by collision in discharge, is of the greatest interest in analytical chemistry. ${ }^{5}$

Complete glow discharge plasmas have eight distinct regions: Aston dark space, cathode layer, cathode dark space, negative glow region, Faraday dark space, positive column, anode dark space, and anode glow region. Analytical chemists focus their interest on two of these regions: the cathode dark space and negative glow region, which can be seen in Figure 1.2.

The cathode dark space is a thin layer, which shows little light emission. Nearly all of the discharge voltage is dropped across this region. The negative glow region, which is field free, extends away from cathode and fills much of the remaining discharge space. It is rich in energetic (fast) electrons, thermalized (slow) electrons, ions, and metastable atoms, so various reactions happen in this region. Optical emission comes from atoms excited by thermalized (slow) electrons, which are generated by inelastic collisions. Ionization is achieved by several mechanisms, among which electron ionization (EI), penning ionization (PI) and charge transfer are 
the most significant. Energetic (fast) electrons are responsible for electron ionization (equation (1)), which is "hard" and essential for maintaining glow discharge. The gas phase neutral particle (M) is ionized and two electrons are generated. One electron is released from collision, and the other is primary electron, which loses energy and becomes a slow electron. Because the potential applied between electrodes can be as high as kilovolts, the EI process in glow discharge is in turn harder than classic (70 or $100 \mathrm{eV})$ EI process.

$$
\mathrm{M}+\mathrm{e}_{\text {fast }}^{-} \rightarrow \mathrm{M}^{+}+2 \mathrm{e}_{\text {slow }}^{-}
$$

Metastable argon atoms, formed by recombination of argon ions and slow electrons (equation (2)), are responsible for Penning ionization (equation (3)), which is a relatively "soft" ionization process. Although the metastable argon population is several orders of magnitude lower than the electron population, they play a disproportionately important role in ionization of sputtered atoms as a result of their long lifetimes. ${ }^{4}$ Previous experiments in our lab showed that Penning ionization is the dominant process in glow discharge and makes up $40-80 \%$ of the total ionization in a continuous glow discharge. ${ }^{8}$ The energy of a metastable argon atom is 11.55 or 11.62 $\mathrm{eV}$, which is bigger than the first ionization potential (IP) of almost all elements. In other words, most of elements can be ionized by the Penning ionization process.

$$
\begin{aligned}
& \mathrm{e}^{-}+\mathrm{Ar}^{+} \rightarrow \mathrm{Ar}^{*} \\
& \mathrm{M}+\mathrm{Ar}^{*} \rightarrow \mathrm{M}^{+}+\mathrm{Ar}+\mathrm{e}^{-}
\end{aligned}
$$


Where Ar* is a metastable argon atom. ${ }^{4}$

When an $\mathrm{Ar}^{+}$collides with another particle (M) in negative glow, an electron may transfer from the particle to $\mathrm{Ar}^{+}$and form $\mathrm{Ar}$ atom and an ion $\left(\mathrm{M}^{+}\right)$. This process is called charge transfer ionization (equation (4)). It is a highly selective ionization process, which is favored when the energy difference between $\mathrm{Ar}^{+}$and product ion is small. Steers and coworkers regarded it as the prominent mechanism to produce excited state ions. ${ }^{11,12}$

$$
\mathrm{M}+\mathrm{Ar}^{+} \rightarrow \mathrm{M}^{+}+\mathrm{Ar}
$$

Based on those ionization processes, only positive ions can be detected in glow discharge and mass spectra is composed of mainly singly charged ions. Some diatomic clusters ions are formed, but only major constituents can be observed such as $\mathrm{Ar}_{2}{ }^{+}$. Discharge gas and water ions are always detected in the spectra. ${ }^{5}$

\subsubsection{Pulsed Glow Discharge}

Glow discharges can operate both in continuous and pulsed power modes. From their name, one can conclude that continuous glow discharge receives a stable power between electrodes and pulsed glow discharge utilizes a modulated power with power on and power off periods. There are three parameters needed to describe a pulsed power supply: peak power, duty cycle, and frequency. Typical pulse parameters used in our lab are 40-120 watts, 5-25\% duty cycle, and 50-100 Hz. 
Pulsed glow discharge is more popular in modern instruments, because it has two distinctive advantages over the continuous mode. On one hand, pulsed glow discharges can supply higher instantaneous power. Continuous cathodic sputtering by high energetic ions and neutrals will accumulate power on cathode and lead it to be overheated, which may cause sample cracking, thermal decomposition, and plasma instabilities. With modulated power, the cathode will be able to cool down during off periods so overheating is minimized. Supplying the same average power within a certain period, the pulsed glow discharge has a higher instantaneous power, which means that the potential and current between cathode and anode will be higher. Higher potential increases kinetic energy of $\mathrm{Ar}^{+}$and results in a two order of magnitude higher sputtering rate than achieved in the continuous mode $;^{9}$ higher current will enhance the ionization of atoms by increasing cross section. Increase of both atomization and ionization gives stronger signal, improves sensitivity, and lowers the detection limit. About 100 times higher signal intensity in the pulsed mode is reported by Harrison's group when the same average power is supplied. ${ }^{10}$

Another advantage is that the pulsed power mode enables time gated ion signal acquisition. Electron ionization, Penning ionization, and charge transfer ionization, co-exist as ionization processes in a continuous glow discharge, but dominate different time regimes and lead to time dependent spectra in the pulsed glow discharge. The first time regime is called the prepeak. The prepeak regime endures for several hundred micro-seconds after initiation of the power pulse. The applied potential accelerates electrons from the cathode toward the anode. Inelastic collisions 
between these accelerated electrons and discharge gas (Ar) produce discharge gas ions $\left(\mathrm{Ar}^{+}\right)$and secondary electrons. The mass spectrum reflects this as it contains signals only for species present in the discharge gas, such as Ar, air and water until the onset of sputtering. Because no sputtered signal is detected, this region is not of analytical interest for sample on cathode. Electron ionization is the dominant process in prepeak regime Sputtering of the sample cathode commences as the population of argon ions increases and accelerates to the cathode surface. It takes about 1 millisecond for the plasma to reach a steady state after power application. At this point, electron impact, charge transfer, and Penning ionization processes all exist in an equilibrium state that remains until the power is removed. This period is analogous to that of continuous glow discharge. Atomic ions from cathodic material, discharge gases, and cluster ions can all be generated. This time regime is called plateau regime. After power termination, the afterpeak regime arises, during which the fast electron population is rapidly thermalized by collisions. The resultant slow electrons recombine with argon ions producing a large population of metastable neutral argon atoms. Electron ionization and charge transfer ionization decrease to trivial level because of vacancy of fast electrons and $\mathrm{Ar}^{+}$. During the afterpeak regime, metastable argon atoms become the dominant ionization agents, providing for Penning ionization of other species in the plasma. The sudden increase of Penning ionization results in a "jump" of signal in spectra. Because this is a soft ionization process, intact molecular ions may be detected as well as atomic ions and molecular fragment ions. High ionization potential stopped ionization of some background species such as argon to decrease 
background noise. Lewis and co-workers made use of this characteristic and successfully analyzed $\mathrm{Ca}^{+}$in the presence of $\mathrm{Ar}^{+}$, both at $\mathrm{m} / \mathrm{z}=40 .{ }^{13}$

\subsubsection{Pulsed Glow Discharge Time-of-Flight Mass Spectrometer (GD-TOFMS)}

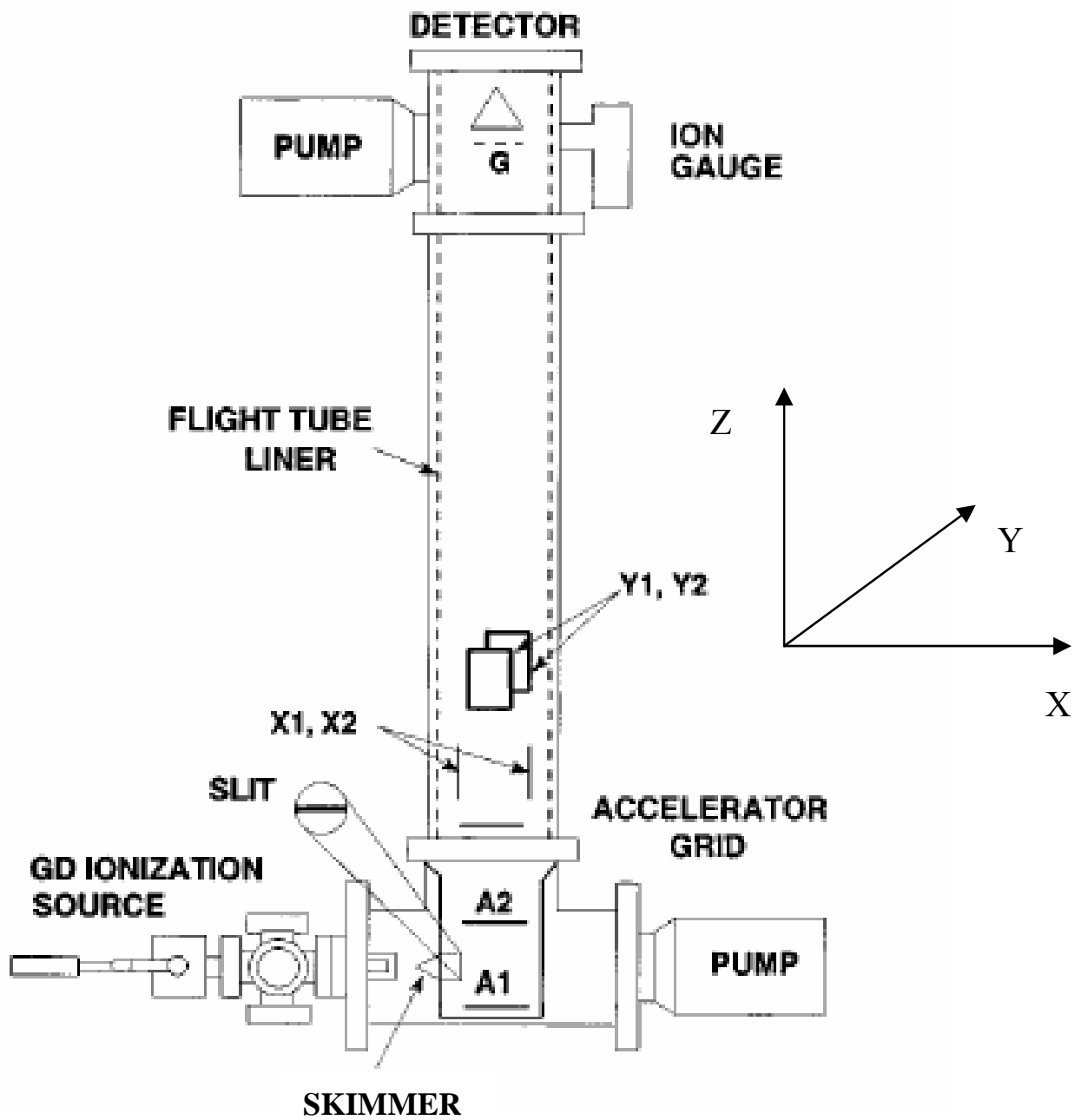

Figure 1.3 Structure of Glow Discharge Time-of-Flight mass spectrometer

A pulsed ion source requires a pulsed sampling mass analyzer. The best and probably easiest match is a Time-of-Flight (TOF) system. Figure 1.3 shows the glow discharge time-of-flight system used in our lab. The detector used here is a dual 
multi-channel plate, which can amplify the ion current signal by up to $10^{6}$ times in magnitude. In this system, ions generated in glow discharge diffuse through skimmer and slit in $\mathrm{x}$ direction and get repelled by microsecond potential pulse applied between plate A1 and A2 towards the flight tube. The potential applied to the ion converts to kinetic energy, $\mathrm{E}_{\mathrm{k}}$, of ions in $\mathrm{Z}$ direction.

$$
\mathrm{E}_{\mathrm{k}}=\mathrm{zV}
$$

where $\mathrm{z}$ is the charge of ion, and $\mathrm{V}$ is the potential applied between plates A1 and A2. Also, kinetic energy

$$
\mathrm{E}_{\mathrm{k}}=m v^{2} / 2
$$

where $\mathrm{m}$ is the mass, $\mathrm{v}$ is velocity of ion in $\mathrm{z}$ direction. By combining these two equations and rearranging them, we get

$$
\mathrm{m} / \mathrm{z}=2 \mathrm{~V} / \mathrm{v}^{2}
$$

where $\mathrm{m} / \mathrm{z}$ is the mass-to-charge ratio.

Because the flight tube is at high vacuum $\left(10^{-6}\right.$ Torr $)$ and is field free in $\mathrm{z}$ direction. The $\mathrm{v}$ is constant for ions of a specific $\mathrm{m} / \mathrm{z}$ while the ions traverse the tube, so the time, $t$, of the ion travels through the tube, whose length is $\mathrm{L}$, is

$$
\mathrm{t}=\mathrm{L} / \mathrm{v}
$$

Rearrange this equation and replace $\mathrm{v}$ value in previous equation to get 


$$
\mathrm{m} / \mathrm{z}=2 \mathrm{Vt}^{2} / \mathrm{L}^{2}
$$

Since $\mathrm{V}$ and $\mathrm{L}$ are the same for each ion, $\mathrm{m} / \mathrm{z}$ value is directly related to $\mathrm{t}$. It is obvious that smaller $\mathrm{m} / \mathrm{z}$ ions will reach detector faster than larger $\mathrm{m} / \mathrm{z}$ ions. What's more interesting is that there is ideally no upper limit to the $\mathrm{m} / \mathrm{z}$ range for time-of-flight mass analyzer. Another advantage of time-of-flight is that it can collect a complete mass spectrum for all ions in one run. For example, $\mathrm{V}$ applied in our instrument is $1800 \mathrm{~V}$, and the length of flight tube is $1 \mathrm{~m}$. The time for a silver ion $(\mathrm{m} / \mathrm{z} 107)$ takes to reach the detector is

$$
\begin{aligned}
& \mathrm{t}=\left((\mathrm{m} / \mathrm{z}) \mathrm{L}^{2} /(2 \mathrm{~V})\right)^{1 / 2} \\
& \left.=\left((107 * 1.7726 \mathrm{E}-27 \mathrm{~kg} / 1.702 \mathrm{E}-19 \mathrm{C})(1 \mathrm{~m})^{2} / 1800 \mathrm{~V}\right)\right)^{1 / 2} \\
& =2.49 \mathrm{E}-5 \mathrm{~s}
\end{aligned}
$$

Similarly, time for a large ion $(\mathrm{m} / \mathrm{z} 10,000)$ to reach the detector can also be calculated, and the result is $2.41 \mathrm{E}-4 \mathrm{~s}$. Therefore TOF can work at very high speed and collect and sum many mass spectra within a short time. Signal to noise ratio $(\mathrm{S} / \mathrm{N})$ is improved by averaging those spectra in proportion to the square root of the number of spectra.

As discussed above, the pulsed glow discharge source offers different ionization processes at different time regimes. In order to properly monitor all chemical species produced during such time regimes occurring in every pulse period, the concurrent 
acquisition of the complete mass spectrum during each process is required. ${ }^{15}$ The rapid speed of TOF spectral acquisition provides this capability perfectly.

Another advantage of TOF instrument is that it affords high ion transmission effect, which results in higher sensitivity, because all the ions generated reach detector. Lange and co-workers reported gramicidin spectra at $10^{-15} \mathrm{~mol}^{15}$ and a 100-200 attomole detection of several proteins was achieved by Onnerfjord's group. ${ }^{16}$

The first GD-TOFMS was built by Harrison's group in $1990 .{ }^{17}$

\subsection{Electrospray - Ion Trap Mass Spectrometry}

\subsubsection{Electrospray Ionization}

Because mass spectrometers separate species apart by their mass-to-charge $(\mathrm{m} / \mathrm{z})$ ratio, all samples must be converted to gaseous ions before entering mass analyzer. This ionization process may be easy for gaseous samples with ion sources such as electronic ionization (EI) or chemical ionization (CI) but difficult for thermally labile, low vapor pressure samples.

Since 1969, scientists have developed several ion sources to solve this problem, such as field desorption (FD), plasma desorption (PD), fast atom bombardment (FAB), liquid secondary ion mass spectrometry (LSIMS), and electrospray ionization (ESI). Most ion sources mentioned above introduce too much internal energy to the sample during ionization process and lead to fragmentation, but ESI, introduced by John Fenn 
in 1989, minimizes fragmentation of thermally labile and large biological samples. In other words, ESI is a very "soft" ionization source. It is also interesting to notice that Fenn's early papers about ESI all apply quadruple as mass analyzers for large protein sample analysis. This is because ions generated in ESI are multiply charged. As a result, a mass analyzer with a low mass range, such as quadrupole mass analyzer, can still be used to analyze large molecules. ${ }^{19,}{ }^{20}$ The higher charge of the ions also increases signal sensitivity at the detector because higher charge will yield greater kinetic energy when accelerated in a constant field. ESI works at atmospheric pressure. After the high pressure difference problem between ESI and mass analyzer is solved by differential pumping system, it is both convenient and attractive to sample at atmospheric pressure. Last, but not least, direct formation of gaseous ions from liquid phase makes ESI suitable to couple with separation techniques like high performance liquid chromatography (HPLC) and capillary electrophoresis (CE). ESI is a "young" ion source, but it is now broadly used in proteomics, polymer and biopolymer, and small polar molecules analysis. John Fenn was awarded Nobel Prize in 2002 to acknowledge his work on ESI. 


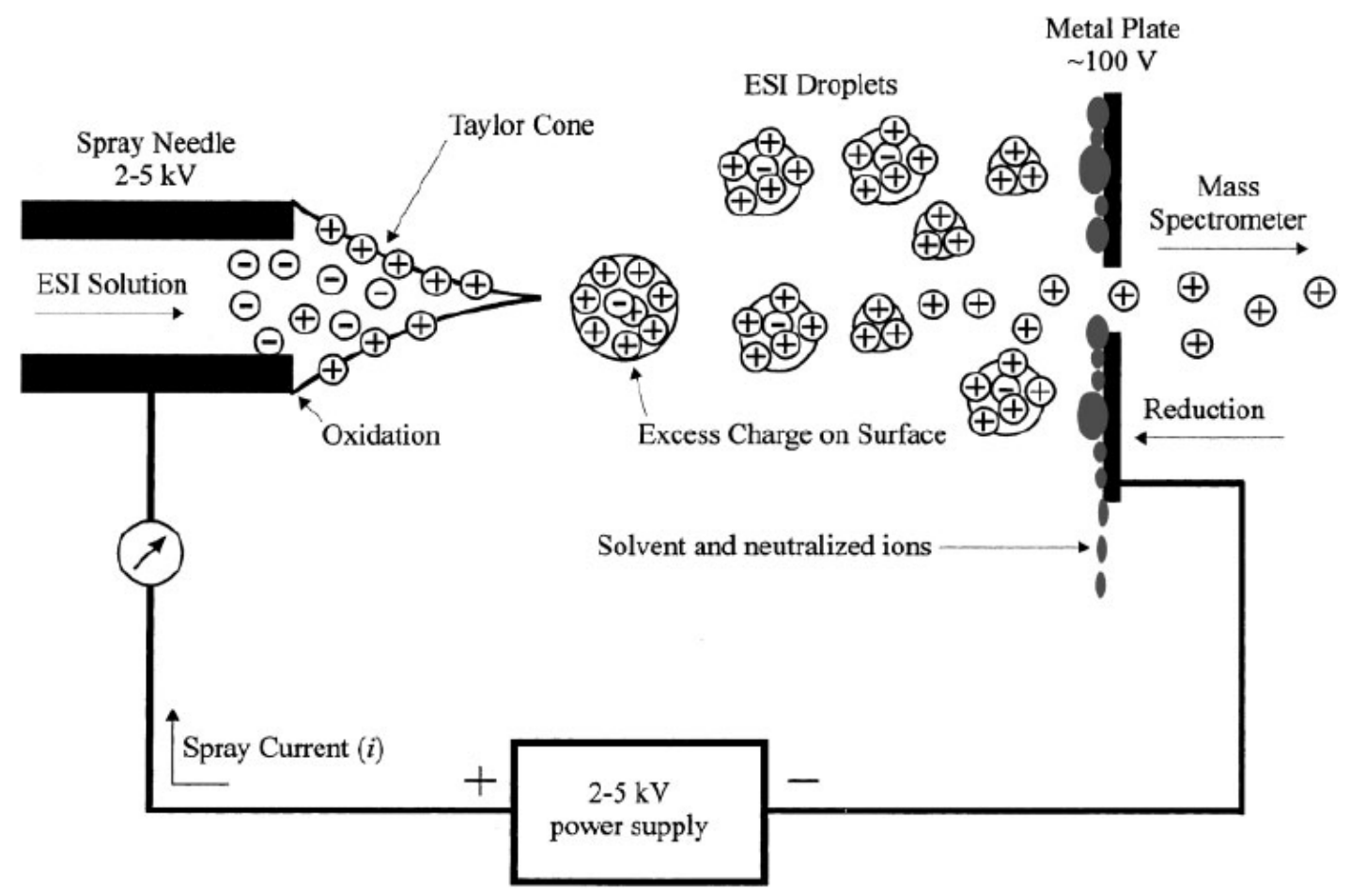

Figure 1.4 Schematic of electrospray ionization process.

Figure 1.4 shows the schematic process of ESI. ${ }^{21}$ A dilute solution of sample is introduced to capillary at a low flow rate $\left(0.1-10 \mu \mathrm{L} \mathrm{min}{ }^{-1}\right)$ while a high voltage $(2-6$ $\mathrm{kV}$ ) is applied between capillary and metal plate. The high voltage can either be positive or negative, depending on the polarity desired of analyte ions. Figure 1.4 shows the case when positive potential is supplied to capillary. The distance between electrode and capillary is $0.3-2 \mathrm{~cm}$ and the electric field between them can reach as high as $10^{6} \mathrm{~V} / \mathrm{m}$. The high potential induces charge accumulation and strong coulombic repulsion at the tip of capillary. The interaction of coulombic repulsion and surface tension drives the liquid to elongate and form a "Taylor cone". As the "Taylor cone" reaches Rayleigh limit, when coulombic repulsion on the surface equals to surface tension, droplets will be generated and spray begins. This potential is called 
the "onset voltage". The Rayleigh limit can be calculated by Rayleigh equation below:

$$
\mathrm{q}^{2}=8 \pi^{2} \varepsilon_{0} \gamma \mathrm{D}^{3}
$$

where $\mathrm{q}$ stands for charge; $\varepsilon_{0}$ is permittivity of the environment; $\gamma$ means surface tension; D is the diameter of a supposed spherical droplet.

A coaxial gas flow is supplied to limit the dispersion of spray and droplets with net positive charge that move toward metal plate as a result of the applied electric field. Two mechanisms are posed regarding molecular ion formation. One is called "coulomb fission mechanism". Based on this mechanism, a droplet shrinks as solvent evaporates, so coulombic repulsion increases as density of charge on the surface increases. A process similar to spray at the capillary happens and a new Taylor cone is formed. When Rayleigh limit is reached, the droplet will break apart and form next generation droplets until single molecular ions are formed. The other mechanism is called "ion evaporation mechanism". It assumes that after evaporation of solvent, ions are directly released from droplet surface when Rayleigh limit is reached. Regardless which mechanism generates the ions, ESI produces gaseous ions directly from the liquid phase to be analyzed in a mass analyzer. ${ }^{21}$

In early ESI source designs, the electrospray and sampling orifice are coaxial as shown in Figure 1.4. Later designs changed this alignment to orthogonal or near orthogonal configuration so that less solvent can reach orifice and only ions can pass 
through orifice. Therefore, a larger orifice can be used and a higher flow rate can be applied. Combination of these factors successfully increases ion transmission and improves sensitivity. ${ }^{5}$

\subsubsection{Quadrupole Ion Trap Mass Analyzer}

Based on ion motion in quadrupolar electric field, W. Paul and H. S. Steinwedel introduced quadrupole mass analyzer in 1953 and described quadrupole ion trap in a patent in $1960 .{ }^{15}$ Paul and Dehmelt were awarded Nobel Prize for their work on quadrupole ion trap in 1989.

Figure 1.5 shows schematic structure of a quadrupole ion trap. There are three electrodes in this tennis ball sized mass analyzer: a rotationally symmetrical ring electrode in the center and two end-cap electrodes besides ring electrode. Ions can enter and leave the trap through orifices on end-cap electrodes. A quadrupole ion trap can be regarded theoretically as a spatial transformation of a quadrupole mass analyzer. Two rods bend and join themselves to form a loop ring electrodes; the other two rods become the end-cap electrodes. ${ }^{5}$ Direct current (DC) and radio frequency (RF) potentials applied to electrodes create a three dimensional (3D) quadrupolar field, which traps all ions inside. Ions can be expelled out of the space by applying an additional RF potential at certain resonant frequency on end-cap electrodes to generate mass spectra. 

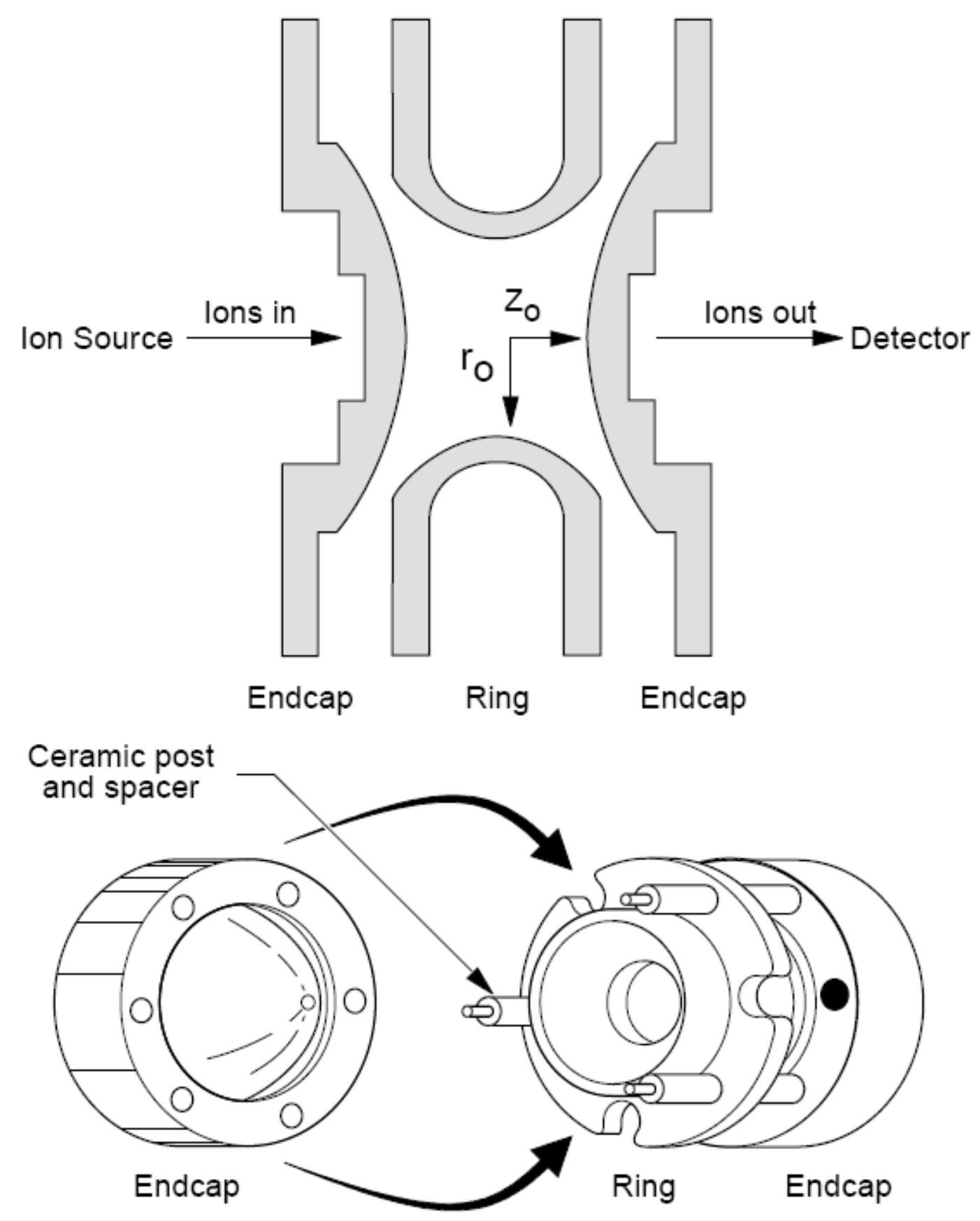

Figure 1.5 Schematic view of quadrupole ion trap. The term $r_{0}$ and $Z_{0}$ are the distance from center to ring electrode and endcap electrode. ${ }^{22}$

Because all ions are trapped, colombic repulsion becomes significant so that ion trajectory will be expanded. Ions will collide with wall of instrument and get neutralized. In order to solve this problem, damping gas such as helium is supplied to 
ion trap. Damping gas collides with ions trapped inside and takes excess kinetic energy away so that ions will be kept in the center of the trap. Typically, the standard ion trap can hold about $10^{6}$ ions. Addition of damping gas also improves the mass resolution of the resulting mass spectra because ions in a smaller region are ejected more efficiently than those in a diffuse ion cloud. ${ }^{24}$ Furthermore, the relative high working pressure $\left(10^{-4}\right.$ Torr) because of damping gas supply makes ion trap an excellent candidate for miniature mass spectrometers, who do not have powerful vacuum pump as bench top machines.

The movement of ions in an ion trap can be described by a Mathieu differential equation, whose general form is shown below:

$$
d^{2} u / d \xi^{2}+\left(a_{u}-2 q_{u} \cos 2 \xi\right) u=0
$$

where $\mathrm{u}$ is the coordinate axes $\mathrm{X}, \mathrm{Y}$, and $\mathrm{Z}$; $\xi$ is a dimensionless parameter equals to $\omega t / 2$ where $\omega$ is frequency and $t$ is time; $a_{u}$ and $q_{u}$ are also dimensionless parameters known as trapping parameters. ${ }^{24}$

Because ions never reach $\mathrm{r}_{0}$ or $\mathrm{Z}_{0}$, a stability area in $\mathrm{Z}$ - direction and $\mathrm{r}$ - direction can be calculated. The result is shown below:

$$
\begin{aligned}
& \mathrm{a}_{\mathrm{Z}}=-2 \mathrm{a}_{\mathrm{r}}=-16 \mathrm{zU} / \mathrm{m}\left(\mathrm{r}_{0}{ }^{2}+2 \mathrm{z}_{0}{ }^{2)} \omega^{2}\right. \\
& \mathrm{q}_{\mathrm{z}}=-2 \mathrm{q}_{\mathrm{r}}=8 \mathrm{zV} / \mathrm{m}\left(\mathrm{r}_{0}{ }^{2}+2 \mathrm{z}_{0}{ }^{2)} \omega^{2}\right.
\end{aligned}
$$

where $\mathrm{a}_{\mathrm{z}}, \mathrm{q}_{\mathrm{z}}, \mathrm{a}_{\mathrm{r}}$, and $\mathrm{q}_{\mathrm{r}}$ are Mathieu stability coordinates in $\mathrm{Z}$ and $\mathrm{r}$ direction; $\mathrm{z}$ is the 
charge number of ion; $\mathrm{U}$ is the direct current voltage applied and $\mathrm{V}$ is the amplitude of radio frequency $(\mathrm{RF})$ voltage applied between ring electrode and end-cap electrodes; $\mathrm{m}$ is the mass number of ion; $\omega$ is the angular frequency which equals to $2 \pi v$, where $v$ is the frequency of RF voltage. Figure 1.6 depicts an ion stability diagram based on $a_{Z}$ and $\mathrm{q}_{\mathrm{Z}}$ values. Because ions have to be stable in both $\mathrm{r}$ - and $\mathrm{Z}$ - directions, overlapping the field of r- direction stability region with the field of Z- direction stability region provides the stable range for ions of a particular $\mathrm{m} / \mathrm{z}$ in a trap.

When too many ions are injected in an ion trap, ions at the outer edge will shield the electric field from ions nearer the center. This effect is called space charge effect. Because the electric field is modified, the stability diagram will change and mass accuracy will be altered. This problem is solved by limiting the number of ions entering the trap during a given analysis by techniques such as gating lenses. 


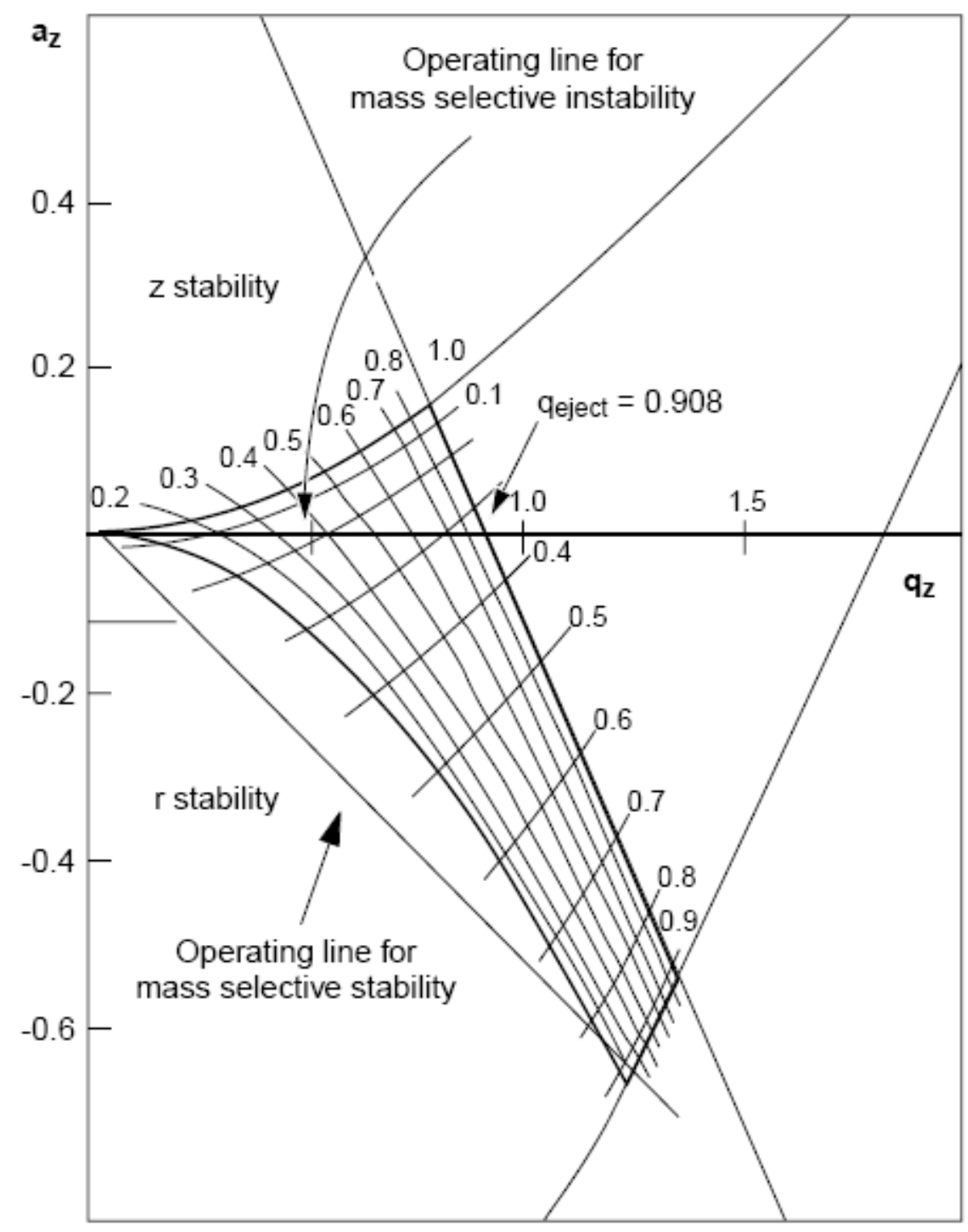

Figure 1.6 Mathieu stability diagram of quadrupole ion trap. Ions are stable in both $r$ and $\mathrm{z}$ direction if its $\mathrm{a}_{\mathrm{z}}$ and $\mathrm{q}_{\mathrm{z}}$ value are in the shaded area. ${ }^{22}$

Because the size of ion trap is set for certain instrument, $r_{0}$ and $Z_{0}$ are constant.

The $\omega$ is also constant in commercial instrument. Therefore, a and $q$ values for specific $\mathrm{m} / \mathrm{z}$ ions are only related to the voltage applied to electrodes. In commercial instruments, an RF only potential is applied to ring electrode and end-cap electrodes are grounded. Therefore, both $\mathrm{V}$ and $\mathrm{q}_{\mathrm{z}}$ values equal to 0 . Under this condition, the 
stability region changes to a line segment along $\mathrm{q}_{\mathrm{z}}$ axis from 0 to 0.908 . For specific $\mathrm{m} / \mathrm{z}$ ion, $\mathrm{q}_{\mathrm{z}}$ value will increase as $\mathrm{V}$ increases based on equation 13 . When the $\mathrm{q}_{\mathrm{z}}$ value reaches boundary, 0.908 , the ion will not be stable along $\mathrm{z}$ direction. It will then exit the trap through orifice on end-cap electrodes and reach detector. From equation $13, \mathrm{~V}$ is proportional to $\mathrm{m} / \mathrm{z}$ value. When $\mathrm{V}$ increases from small to high value, small ions will be ejected out first and get detected. Higher mass ions are expelled and detected later. By this operation, a mass spectrum of all ions will be generated via a mass selective instability scan. ${ }^{26,27}$

The mass range of a mass selective instability scan is limited by the amplitude of RF voltage supplied to ring electrode, because too high of a voltage will cause electrical breakdown of analytes. This problem was solved by applying a RF potential to the endcaps as well. Trapped ions have a characteristic frequency of oscillation in both r- and Z- direction called secular frequencies. When an RF potential with the certain frequency is supplied, the ion whose secular frequency is the same with that of $\mathrm{RF}$ potential will gain more kinetic energy. If the $\mathrm{RF}$ potential is applied along $\mathrm{Z}$ axis, in other words on end-cap electrodes, ions will be activated along $\mathrm{Z}$ direction by resonance excitation. If the potential applied to end-cap electrodes are not high enough, ions will not be ejected out of trap but have a broader trajectory, which results in more collisions with damping gas (Helium). The collision may lead to fragmentation of ions and this process is regarded as collision induced dissociation (CID). CID can be used to acquire structure information of molecular ions. 
Tandem mass spectrometry is a procedure to examine specific ions in a mixture of ions. The target ions are separated based on $\mathrm{m} / \mathrm{z}$ value, and then undergo collisions that result in fragmentation. The fragment ions are analyzed in mass spectrometer again. There are two kinds of tandem mass spectrometry. One is called tandem mass spectrometry in space or MS/MS; the other is called tandem mass spectrometry in time or $\mathrm{MS}^{\mathrm{n}}$. Triple quadrupole is a classical and successful tandem mass spectrometry in space; whereas the quadrupole ion trap together with other ion trapping instruments, such as orbitrap and ion cyclotron resonance trap, provide tandem mass spectrometry in time.

To perform tandem mass analysis in an ion trap mass spectrometer, a first generation ion is selected and all other ions are ejected out of trap. The selected ion is activated by RF voltage applied to end-cap electrodes as discussed above. Collision between motivated ion and damping gas molecules leads to CID process. Product fragments can be analyzed by mass selective instability scan. The main advantage of tandem mass analysis is enhanced speciation with the help of fragment ion information. It can also reduce matrix signals and instrumental noise. The most attractive factor of $\mathrm{MS}^{\mathrm{n}}$ is theoretically unlimited fragmentation of sample ions. After first time fragmentation of target ions, fragment ions can be further isolated and fragmented again. More structure information can be acquired. $\mathrm{MS}^{\mathrm{n}}$ supplies a fast and easy way for structure elucidation. 


\subsection{References}

1. Martin S. Silberberg. Principles of General Chemistry; McGraw-Hill Companies, Inc.: New York, 2007.

2. Mortimer, Charles E. Chemistry: A Conceptual Approach (3rd ed.); D. Van Nostrad Company: New York, 1975.

3. Brian Chapman. Glow Discharge Process; John Wiley \& Sons Inc.: New York, 1980.

4. Harrison, W. W.; Hess, K. R.; Marcus, R. K.; King, F. L., Glow Discharge Mass Spectrometry. Analytical Chemistry, 1986, 58(2), 341A-352A.

5. Hoffmann, E.; Stroobant, V., Mass Spectrometry: Principles and Applications, John Wiley and Sons: West Sussex, 2002.

6. Klingler, J. A.; Harrison, W. W. Proceedings of the 39th ASMS Conference on Mass Spectrometry and Allied Topics; Nashville, TN, May 19-24, 1991; ASMS: Sante Fe, NM, 1991; p 943.

7. R. Kenneth Marcus, Glow Discharge Spectroscopies, Plenum Press: New York, 1993.

8. Pan, C.; King, F. L., Temporal Signal Profiles of Analytical Species in Modulated Glow Discharge Plasma. Anal. Chem. 1993, 65, 3107-3193.

9. Pollmann, D., Ingeneri, K.; Harrison, W. W., Comparison of Atomization and Ionization Processes in Direct Current, Radiofrequency and Microsecond Pulse Discharges. J. Anal. At. Spectrom. 1996, 11, 849.

10. Walden,W. O.; Harrison, W. W.; Smith, B. W.; Winefordner, J. D., Multi-element 
Glow Discharge Atomic Fluorescence Using Continuum Sources. J. Anal. At. Spectrom. 1994, 9, 1039-1043.

11. E. B. M. Steers and R. J. Fielding, Charge-transfer Excitation Processes in the Grimm Lamp. J. Anal. At. Spectrom. 1987, 2, 239.

12. E. B. M. Steers and F. Leis, Observations on the Use of the Microwave-boosted Glow Discharge Lamp and the Relevant Excitation Processes. J. Anal. At. Spectrom. 1989, 4, 199.

13. Cris L. Lewis, Eric S. Oxley, Charles k. Pan, Robert E. Steiner, and Fred L. King., Determination of ${ }^{40} \mathrm{Ca}^{+}$in the Presence of ${ }^{40} \mathrm{Ar}^{+}$: an Illustration of the Utility of Time-gated Detection in Pulsed Glow Discharge Mass Spectrometry. Anal. Chem. $1999,71,230-234$.

14. Rosario Pereiro, Auristela Solà-Vázquez, Lara Lobo, et al. Present and Future of Glow Discharge — Time of Flight Mass Spectrometry in Analytical Chemistry. Spectrochimica Acta Part B 66 (2011) 399-412.

15. W. Lange, D. Greifendorf, D. Van Leyen, et al. Direct Quantification of Cocaine in Urine by Time-of-flight Mass Spectrometry. Springer Pro. Phys. 1986, 9, 67

16. P. Onnerfjord, J. Nilsson, L. Wallman, et al. Picoliter Sample Preparation in MALDI-TOF MS Using a Micromachined Silicon Flow-Through Dispenser. Anal. Chem. 1998, 70, 4755-60.

17. J.A. Klinger, P.J. Savickas, W.W. Harrison, The Pulsed Glow Discharge as an Elemental Ion Source. J. Am. Soc. Mass Spectrom. 1 (1990) 138-143.

18. M. Mann, C. K. Meng, J. B. Fenn., Interpreting Mass Spectra of Multiply Charged 
Ions. Anal. Chem. 1989, 61, 1702-8.

19. J. B. Fenn, G. J. Van Berkel, C. G. Enke., Electrospray Ionization for Mass Spectrometry of Large Biomolecules. Science, 246, 64.

20. Nadja B. Cech, Christie G. Enke., Practical Implications of Some Recent Studies in Electrospray Ionization Fundamentals. Mass Spectrometry Reviews, 2001, 20, $362-387$.

21. W. Paul and H. Steinwedel, High-pressure Ion Trap Mass Spectrometry. US Pat. 2 $939952(1960)$.

22. Philip S.H. Wong, R. Graham Cooks., Ion Trap Mass Spectrometry. Current Separation. 1997, 16, 85-92.

23. Yuchen $\mathrm{Lu}$, Mass Spectrometric Investigation of Metallothionein and Ionic Liquids Using Electrospray Ion Source Coupled with Quadrupole Ion Trap Mass Analyzer, West Virginia University Libraries: Morgantown 2006.

24. Raymond E. March, Ion Trap Mass Spectrometry. J. Mass Spectrom. 1992, 118-119, 71-135.

25. R. G. Cooks, G. L. Glish, S. A. McLuckey and R.E. Kaiser, Ion Trap Mass Spectrometry. Chem.Eng. News, (1991) 69(12), 26-41.

26. Arseniev, A.; Schultze, P.; Worgotter, E.; Braun, W.; Wagner, G.; Vašák, M.; Kagi, J. H. R.; Wuthrich, Crystallization and Preliminary X-ray Study of AaH IT2, an Insect-specific Toxin from the Scorpion Androctonus Australis Hector. K. J. Mol. Biol. 1988, 214, 637-657. 


\section{Chapter 2}

\section{Internal Energy Distribution of Collision Induced Dissociation in Quadrupole Ion Trap Mass Spectrometry}

\subsection{Introduction}

Different ion sources in mass spectrometry will generate different mass spectra based on internal energy distributions, $\mathrm{P}(\mathrm{E})$, of precursor ions, the energetic barrier of the unimolecular reaction, and the time window corresponding to the observation of the dissociation process. Electron ionization (EI) supplies a precisely fixed high kinetic energy to electrons (typically $70 \mathrm{eV}$ ), which causes electronic and vibrational excitation of molecular species. The $\mathrm{P}(\mathrm{E})$ of generated ions in $\mathrm{EI}$ is quite reproducible whatever instruments are used due to the low pressure of the source and the ion acceleration zone. ${ }^{1}$ The EI spectra can therefore be compiled to form databases suitable for library searching. The limitation of EI lies in requirement of volatile samples and sometimes the absence of molecular ions because of extensive fragmentation. On the contrary, "soft" ionization methods such as electrospray ionization $(\mathrm{ESI})^{2,3}$ and matrix-assisted laser desorption/ionization (MALDI) ${ }^{4,5}$ can produce gas phase molecular or quasi-molecular ions from low volatility samples and labile compounds. The lack of fragmentation in these sources, however, leads to difficulty in structure characterization, which is important for large biological 
molecules. Tandem mass spectrometry successfully solved this problem using ion activation techniques such as collision induced dissociation (CID). By controlling amount of energy introduced to promote the fragmentation process, optimization of selective ion abundances and isomer differentiation could be achieved. ${ }^{6-9}$

There are a few methods for internal energy measurements in mass spectrometry. The "Survival Yield" method developed by De Pauw and co-workers has been used in $\mathrm{P}(\mathrm{E})$ measurement of several ion sources, including electrospray ionization (ESI), fast atom bombardment (FAB), liquid secondary ion mass spectrometry (LSIMS), matrix-enhanced laser desorption/ionization (MELDI), and matrix-assisted laser desorption/ionization (MALDI). ${ }^{10-17}$ This method assumes that the amount of internal energy of the molecular ions and the fragmentation barrier determines the degree of fragmentation. Cooks came up with another method to measure $\mathrm{P}(\mathrm{E})$ by using "thermometer molecules", whose fragmentation pathway consists of several consecutive reactions with known activation energies. This technique has been used to study EI, low energy and high energy collision induced dissociation (CID) in triple quadrupole (QqQ) and Fourier transform mass spectrometry (FTMS). ${ }^{18-21}$ The fragmentation pathway of "thermometer ions" is shown below:

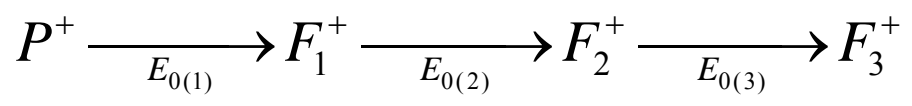

Where $\mathrm{P}^{+}$is the precursor ion, $\mathrm{F}_{\mathrm{n}}^{+}(\mathrm{n}=1,2,3)$ are fragment ions, $\mathrm{E}_{0(\mathrm{n})}(\mathrm{n}=1,2$, 3) are activation energy required to form fragment ions. A plot of $\mathrm{P}(\mathrm{E})$ versus internal energy is generated by using average of adjacent activation energy as $\mathrm{x}$ value 
$\left(\left[\mathrm{E}_{\mathrm{n}}+\mathrm{E}_{\mathrm{n}+1}\right] / 2\right)$ and fragment ion intensity divided by activation energy interval as $\mathrm{y}$ value $\left(\left[F_{n}\right] /\left(E_{n+1}-E_{n}\right)\right)$. This method neglects all effects of kinetic factors and assumes fragmentation to happen as long as the internal energy of ions exceeds the activation energy. Metastable ions are also neglected. These assumptions are valid for instruments with slower flight times such as quadrupoles and ion trapping systems, where the time scale of observation is significantly beyond that for metastable dissociation.

Different from the CID process in triple quadrupole mass spectrometry, the kinetic energy of the precursor ions in the commercial ion trap mass spectrometry system (Thermo LCQ) is ambiguous. Therefore the maximum internal energy $\left(\mathrm{E}_{\mathrm{cm}}\right)$ deposited in the precursor ions after CID cannot be directly calculated. What's more, the target gas (Helium) used in the CID process is light, which means that little internal energy is deposited in precursor ions after each collision. Last but not least, the pressure in the ion trap is relative high $\left(10^{-4}\right.$ torr $)$ and multiple collisions lead to the CID process. High pressure is known to lead to dissociation of fragment ions and multiple collisions will cause larger energy deposition. ${ }^{19}$ All these factors indicate that $\mathrm{P}(\mathrm{E})$ in this CID process is different from other techniques that have been investigated above. The value of relative collision energy provided in LCQ software is more like a qualitative guide instead of a quantitative value.

Temporal and spatial $\mathrm{P}(\mathrm{E})$ of a millisecond pulsed radio frequency glow discharge was measured by Li and co-workers by using the "thermometer molecule" 
method in our lab before. ${ }^{22} \mathrm{P}(\mathrm{E})$ of several ionization methods at different positions within the plasma and at different times during the pulse cycle were successfully measured. Based on this finding we decided to explore the use of triethyl phosphate as a "thermometer molecule" to better define $\mathrm{P}(\mathrm{E})$ of the CID process in tandem mass analysis of a quadrupole ion trap mass spectrometry.

\subsection{Experimental}

Triethyl phosphate (+99\%) was purchased from Alfa Aesar (Ward Hill, MA). Methanol (HPLC grade), and acetic acid (analytical grade) were obtained from Fisher Scientific (Pittsburgh, PA). All chemicals were used directly without further purification. Deionized water was used throughout the experiments. Triethyl phosphate is prepared as $10 \mu \mathrm{M}$ solution by dissolving the solute in methanol, deionized water, and acetic acid (49:49:1, v:v:v) solvent.

ESI-MS analyses of triethyl phosphate were carried out on a quadrupole ion trap mass spectrometer coupled with a standard electrospray ionization source (Thermo LCQтм, San Jose, CA). The heated metal capillary was maintained at 200 ${ }^{\circ} \mathrm{C}$. The spray voltage was set to $4.5 \mathrm{kV}$. Nitrogen was used as the sheath gas and its flow rate was 30 units/min. The capillary voltage and the tube lens voltage were held at $35 \mathrm{~V}$ and $10 \mathrm{~V}$, respectively. Pressure in ion trap is $2 * 10^{-4}$ Torr. The triethyl phosphate solutions were introduced by direct infusion with a flow rate of $5 \mu \mathrm{L} / \mathrm{min}$.

\subsection{Result and Discussion}


Figure 2.1 shows tandem mass spectra of triethyl phosphate quasi-molecular ion $(\mathrm{m} / \mathrm{z} 183)$ collected at different relative collision energies (\%CID). The first fragment ion $\left(\mathrm{F}_{1}{ }^{+}, \mathrm{m} / \mathrm{z} 155\right)$ did not show up until the relative collision energy reached $7 \%$. This indicates that the internal energy deposited to the precursor ions reached $1.4 \mathrm{eV}$ (Table 2.1). The second fragment ion $\left(\mathrm{F}_{2}^{+}, \mathrm{m} / \mathrm{z}\right.$ 127) began to appear when the relative collision energy increased to $8 \%$, which correspond to $2.6 \mathrm{eV}$ deposited to the precursor ions. The third fragment ion $\left(\mathrm{F}_{3}{ }^{+}, \mathrm{m} / \mathrm{z} 99\right)$ became observable when the relative collision energy is $11 \%$. The internal energy required to generate this ion is $4.2 \mathrm{eV}$. As Relative collision energy increased, smaller fragment ions accounted for more in of the total ions observed. The fourth fragment ion $\left(\mathrm{F}_{4}{ }^{+}, \mathrm{m} / \mathrm{z} 81\right)$, which needs 7.7 eV energy to be deposited in the precursor ions, was not observed even as the relative collision energy reached $50 \%$. At relative collision energy values above $50 \%$, significant spectral interferences were observed arising from self reaction of the fragment and precursor ions. 
(a) $8 \%$ CID

8\%CID_sample-10micromol_070606150710_8\% CID \#1 RT: 0.03 AV: 1 NL: 8.92E4

T: + p Füll ms2 183.10@cid8.00 [50.00-500.00]

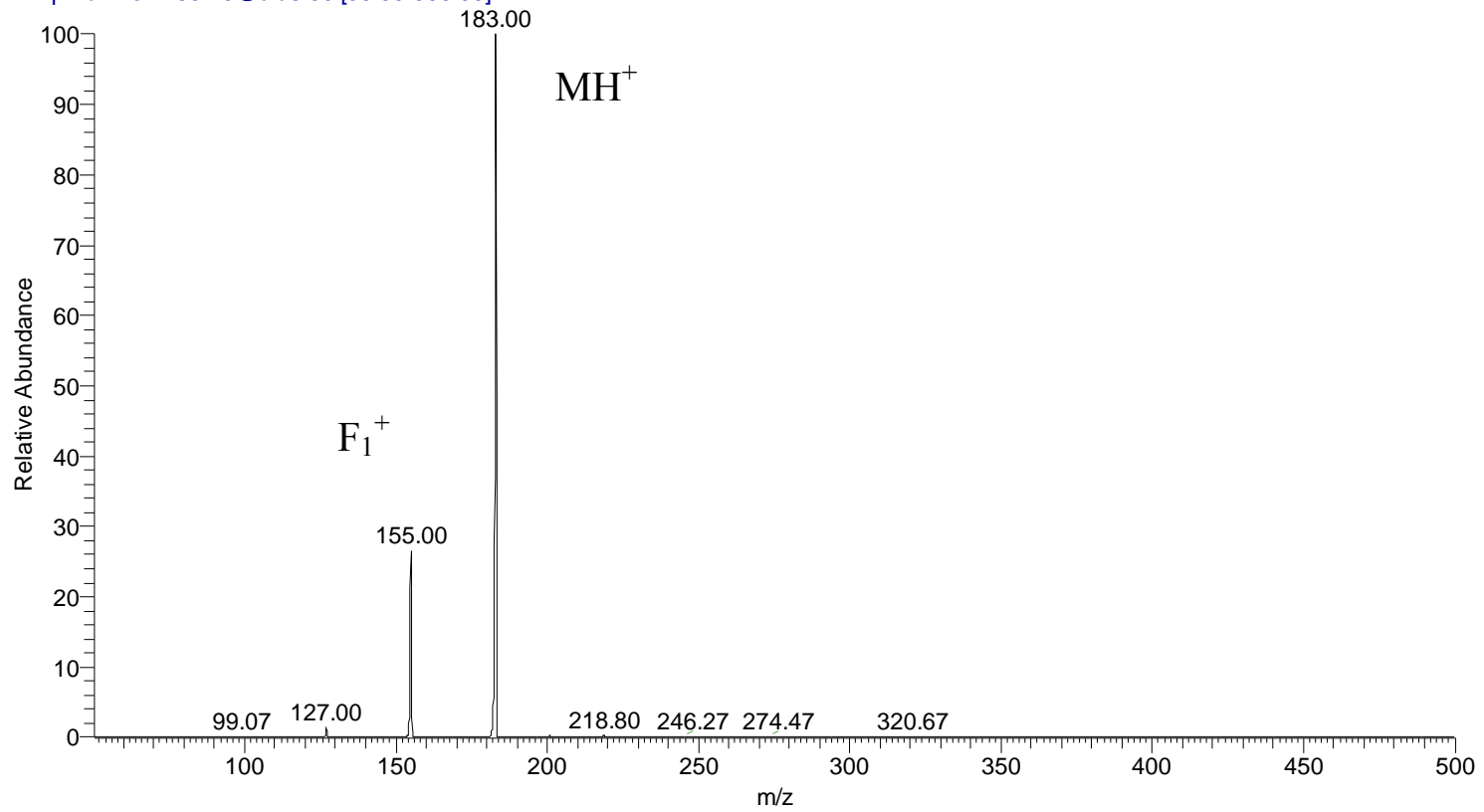

(b) $10 \% \mathrm{CID}$

10\%CID_sample-10micromol_0706061515 \#1 RT: 0.01 AV: 1 NL: 8.50E4

T: + p Full ms2 183.10@cid10.00 [50.00-500.00]

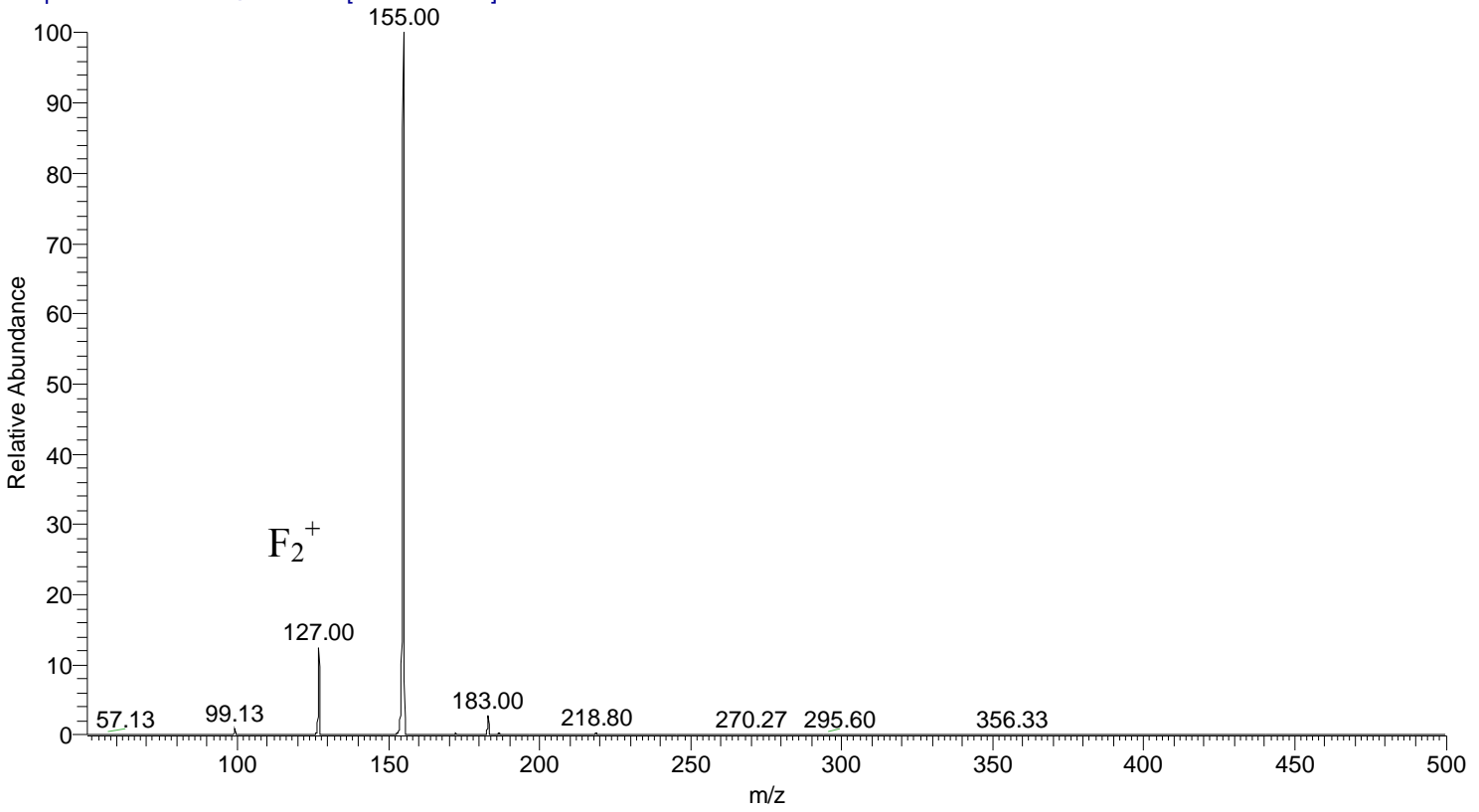


(c) $20 \%$ CID

20\% CID_sample-10micromol_0706061538\#1 RT: 0.01 AV: 1 NL: 3.16E4

T: + p Full ms2183.10@cid20.00 [50.00-500.00]

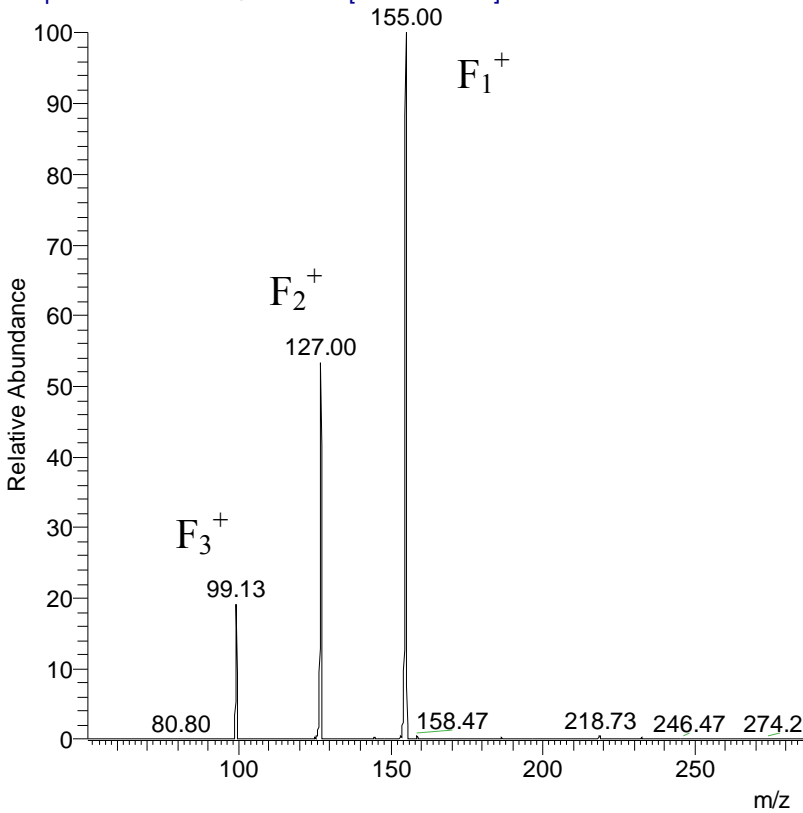

(d) $30 \%$ CID

30\% CID sample-10micromol 0706061435 \#1 RT: 0.03 AV: 1 NL: 4.22E3

T: + p Full ms2 183.10@cid30.00 [50.00-500.00]

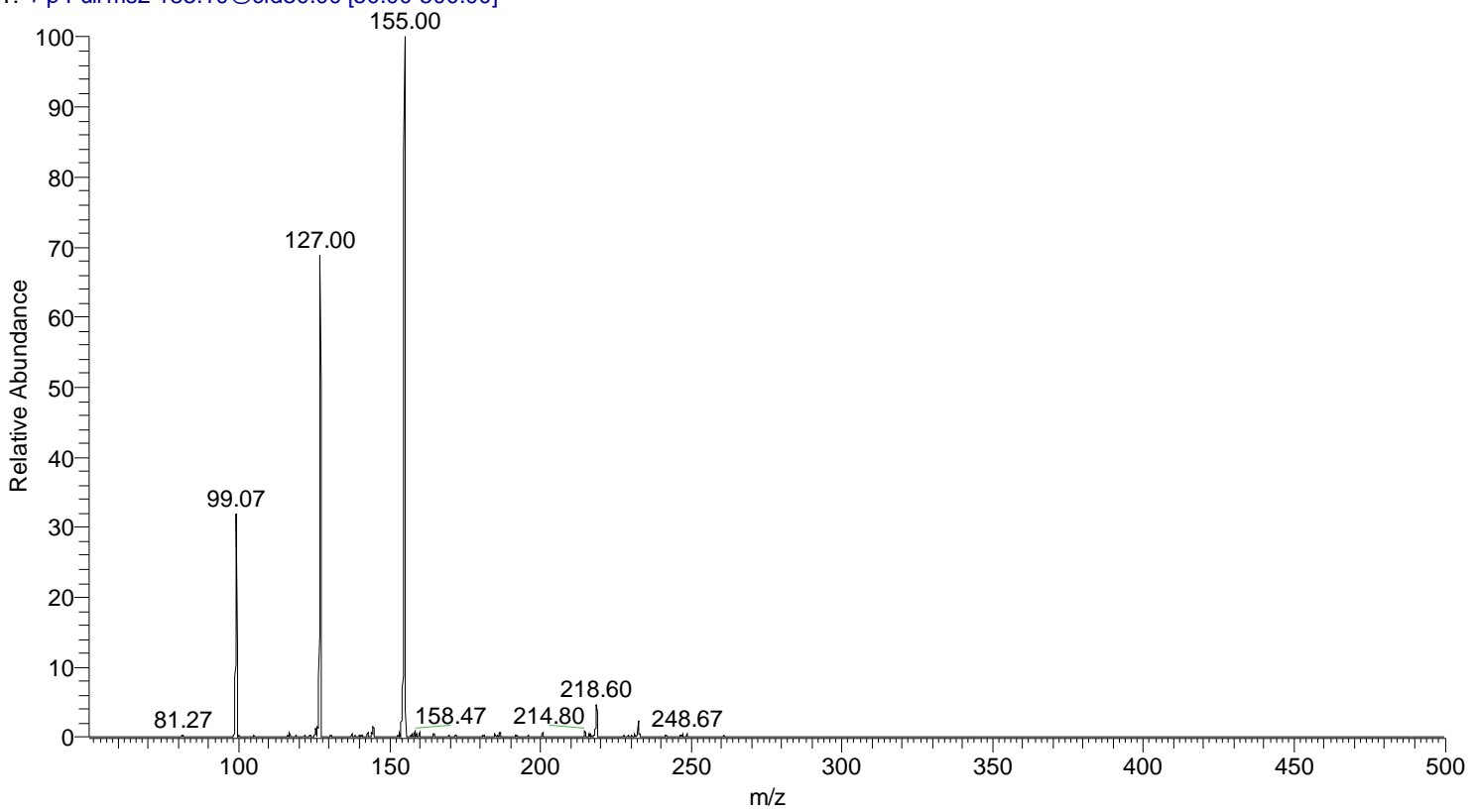

(f) $40 \%$ CID 


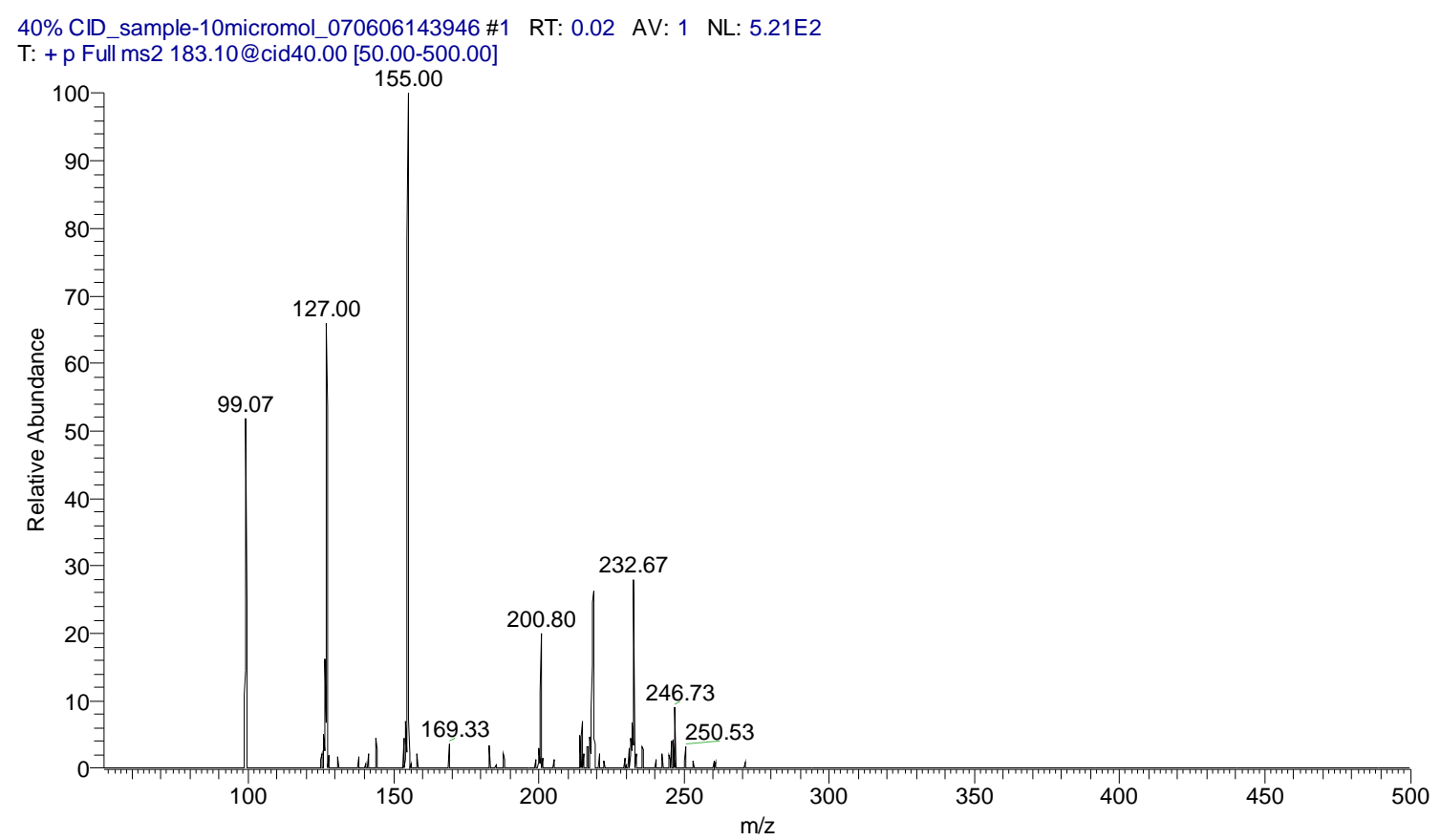

Figure 2.1 Tandem mass spectra of the $\left(\mathrm{C}_{2} \mathrm{H}_{5} \mathrm{O}\right)_{3} \mathrm{PO} \cdot \mathrm{H}^{+}$ion at (a) $8 \% \mathrm{CID}$, (b) $10 \%$ CID, (c) $20 \% \mathrm{CID}$, (d) $30 \% \mathrm{CID}$, (e) $40 \% \mathrm{CID}$ in a quadrupole ion trap mass spectrometry.

The $\mathrm{P}(\mathrm{E})$ of the CID process is plotted in Figure 2.2 based on the mass spectra collected at different relative collision energy and thermochemical value of triethyl phosphate shown in Table 2.1. Based on the figure, the internal energy deposited to the precursor ions rises up as the relative collision energy increases. Note that when the relative collision energy increased from $11 \%$ to $40 \%$, the internal energy deposited to the precursor ions did not change much and the maximum value observed was below $7.7 \mathrm{eV}$. This indicates that there is a limit of internal energy that can be deposited to the precursor ions in this activation method. It also confirms the assumption that the relative collision energy value is not a quantitative value but rather a qualitative guidance parameter. 
Table 2.1 Thermochemical data of triethyl phosphate.

\begin{tabular}{|c|c|c|c|}
\hline Ion & $\mathrm{m} / \mathrm{z}$ & $\mathrm{IE} / \mathrm{eV}^{23}$ & $\mathrm{E}_{0} / \mathrm{eV}^{18}$ \\
\hline$\left(\mathrm{C}_{2} \mathrm{H}_{5} \mathrm{O}\right)_{3} \mathrm{PO} \cdot \mathrm{H}^{+}$ & 183 & 10.0 & 0 \\
\hline$\left(\mathrm{C}_{2} \mathrm{H}_{5} \mathrm{O}\right)_{2} \mathrm{PO}_{2} \mathrm{H}_{2}{ }^{+}$ & 155 & 11.4 & 1.4 \\
\hline$\left(\mathrm{C}_{2} \mathrm{H}_{5} \mathrm{O}\right) \mathrm{PO}_{3} \mathrm{H}_{3}{ }^{+}$ & 127 & 12.6 & 2.6 \\
\hline $\mathrm{PO}_{4} \mathrm{H}_{4}^{+}$ & 99 & 14.2 & 4.2 \\
\hline $\mathrm{PO}_{3} \mathrm{H}_{2}^{+}$ & 81 & 17.7 & 7.7 \\
\hline
\end{tabular}

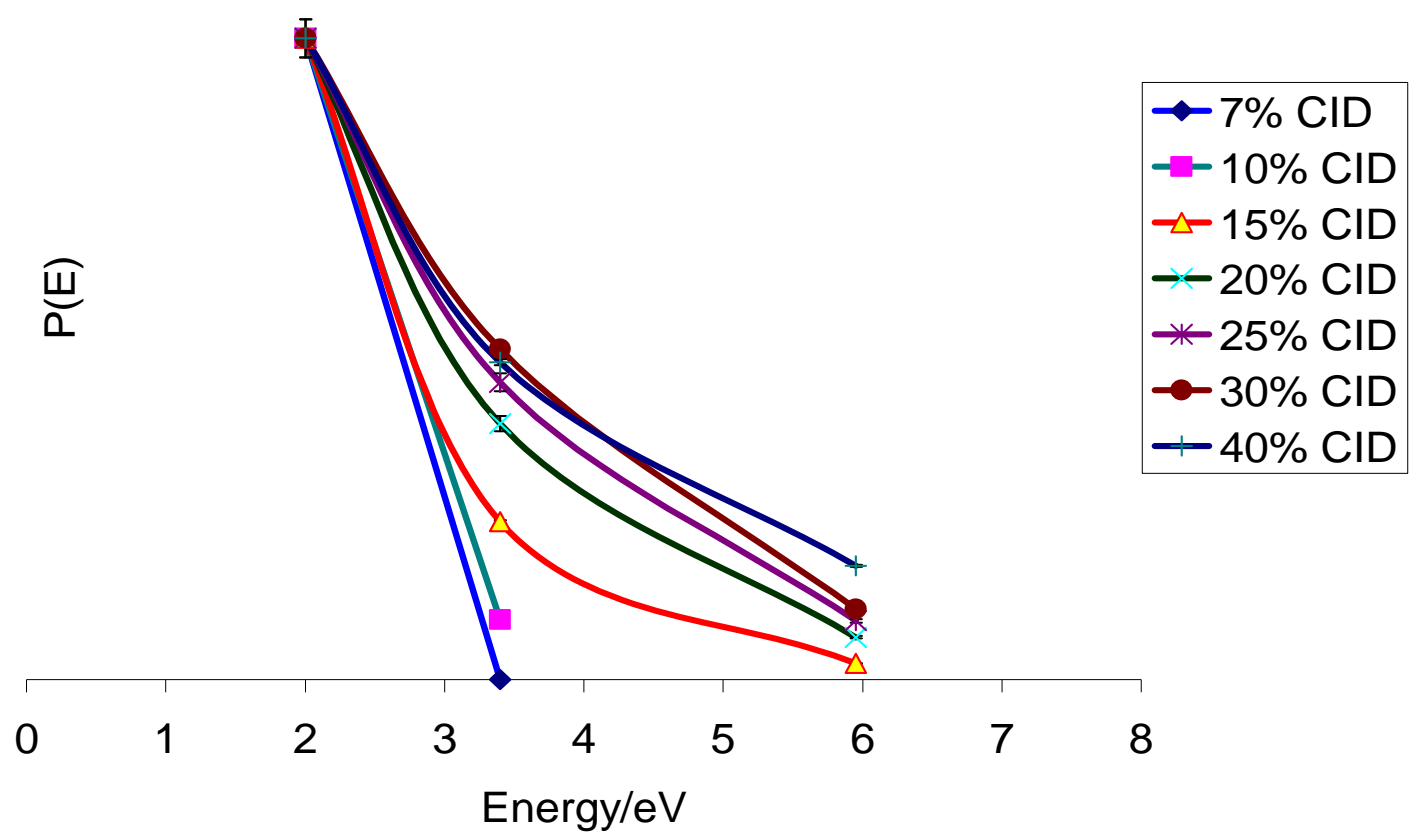

Figure 2.2 $\mathrm{P}(\mathrm{E})$ of the $\left(\mathrm{C}_{2} \mathrm{H}_{5} \mathrm{O}\right)_{3} \mathrm{PO} \cdot \mathrm{H}^{+}$ion activated by $\mathrm{CID}$ in a quadrupole ion trap mass spectrometry. Each data point is the result of 3 measurements and error bars represent one standard deviation.

The $\mathrm{P}(\mathrm{E})$ of the CID process is plotted in Figure 2.2 based on the mass spectra collected at different relative collision energy and thermochemical value of triethyl phosphate shown in Table 2.1. Based on the figure, the internal energy deposited to the precursor ions rises up as the relative collision energy increases. Note that when the relative collision energy increased from $11 \%$ to $40 \%$, the internal energy deposited 
to the precursor ions did not change much and the maximum value observed was below $7.7 \mathrm{eV}$. This indicates that there is a limit of internal energy that can be deposited to the precursor ions in this activation method. It also confirms the assumption that the relative collision energy value is not a quantitative value but rather a qualitative guidance parameter.

The observation of multiple collisions happening at high pressure and further fragmentation of fragment ions was reported in literature. In this experiment, however, the absence of the third fragment $\left(\mathrm{F}_{3}{ }^{+}, \mathrm{m} / \mathrm{z}\right.$ 81) at the high relative collision energy demonstrates that the excitation of fragment ions arising from multiple collisions is quite limited.

\subsection{Conclusion.}

This experiment explored the measurement of internal energy distributions, $\mathrm{P}(\mathrm{E})$, of the CID process in a quadrupole ion trap mass spectrometry by using the "thermometer molecule" method. When the relative collision energy parameter changes from $1 \%$ to $40 \%$, deposited internal energy in the precursor ions is found to increase continuously as expected. The absence of fragment ion $(\mathrm{m} / \mathrm{z} 81)$ not only indicates that there is a limit of deposited energy to the precursor ions, which is smaller than $7.7 \mathrm{eV}$, but shows that multiple collision induced dissociation contributes minimally. Higher relative collision energies appeared to initiate self reaction among precursor and fragment ions. 


\subsection{References}

1. K. Vekey, J. Mass Spectrom., Internal Energy Effects in Mass Spectrometry. 1996, $31,445-63$.

2. M. Yamashita, J.B. Fenn, Electrospray Ion Source. Another Variation on the Free-Jet Theme. J. Phys. Chem., 1984, 88, 4451.

3. M. Whitehouse, R.N. Dreyer, M. Yamashita, J.B. Fenn, Electrospray Interface for Liquid Chromatographs and Mass Spectrometers. Anal. Chem., 1985, 57, 675.

4. M. Karas, D. Bachmann, U. Bahr, F. Hillenkamp, Matrix-assisted Ultraviolet Laser Desorption of Non-volatile Compounds. Int. J. Mass Spectrom. Ion Process., $1987,78,53$.

5. M. Karas, F. Hillenkamp, Laser Desorption Ionization of Proteins with Molecular Masses Exceeding 10,000 Daltons. Anal. Chem., 1988, 60, 2299.

6. D.P. Stevenson and C.D. Wagner, Mass Spectrometric Analysis of Low-molecular-weight Monodeutero-paraffins. J. Am. Chem. Sot., 1950, 72, 5612.

7. F.H. Field and S.H. Hasting, Determination of Unsaturated Hydrocarbons by Low Voltage Mass Spectrometry. Anal. Chem., 1956, 28, 1248.

8. J. Sunner and I. Szabo, Charge Transfer Mass Spectra of Isomers I. n- $\mathrm{C}_{4} \mathrm{H}_{10}$ and i- $\mathrm{C}_{4} \mathrm{H}_{10}$. Int. J. Mass Spectrom. Ion Phys., 1977, 25, 241.

9. A.N.H. Yeo and D.H. Williams, Variation of Metastable Ion Abundance Ratios with Internal Energy in the Mass Spectrometer. J. Am. Chem. Sot., 1971, 93, 395

10. Collette C, De Pauw E. Calibration of the Internal Energy Distribution of Ions Produced by Electrospray. Rapid Communications in Mass Spectrometry, 1998, 
12,165 .

11. Collette C, Drahos L, De Pauw E, V'ekey K. Comparison of the Internal Energy Distributions of Ions Produced by Different Electrospray Sources. Rapid Communications in Mass Spectrometry, 1998, 12, 1373.

12. Drahos L, Heeren RMA, Collette C, De Pauw E, V'ekey K. Thermal Energy Distribution Observed in Electrospray Ionization. Journal of Mass Spectrometry 1999, 34, 1373.

13. Gabelica V, De Pauw E, Karas M. Influence of the Capillary Temperature and the Source Pressure on the Internal Energy Distribution of Electrosprayed Ions. International Journal of Mass Spectrometry 2004, 231, 189.

14. Williams DH, Naylor S. The Internal Energy Distribution in Fast Atom Bombardment/Liquid Secondary Ion Mass Spectra. Journal of the Chemical Society, Chemical Communications 1987, 18, 1408.

15. Derwa F, De Pauw E, Natalis P. New Basis for a Method for the Estimation of Secondary Ion Internal Energy Distribution in Soft Ionization Techniques. Organic Mass Spectrometry 1991; 26: 117.

16. Luo G, Marginean I, Vertes A. Internal Energy of Ions Generated by Matrix-Assisted Laser Desorption/Ionization. Anal. Chem., 2002, 74, 6185.

17. Greisch JF, Gabelica V, Remacle F, De Pauw E. Thermometer Ions for Matrix-Enhanced Laser Desorption/Ionization Internal Energy Calibration. Rapid Communications in Mass Spectrometry 2003, 17, 1847.

18. H. I. Kenttamaa, G. R. Cooks, Internal energy distributions acquired through 
collisional activation at low and high energies. International Journal of Mass Spectrometry and Ion Processes, 1985, 64, 79-83.

19. V. H. Wysocki, H. I. Kenttamaa, G. R. Cooks, Internal energy distributions of isolated ions after activation by various methods. International Journal of Mass Spectrometry and Ion Processes, 1987, 75, 181-208.

20. R.B.Cody, R.C. Burnier, B.S. Freiser, Collision-Induced Dissociation with Fourier Transform Mass Spectrometry. Anal. Chem., 1982, 54, 96.

21. L. Sallens, K.R. Lane, R.R. Squires, B.S. Freiser, Generation and reaction of atomic metal anions in the gas phase. Determination of the heterolytic and homolytic bond energies for the vanadium, chromium, iron, cobalt, and molybdenum hydrides VH, CrH, FeH, CoH, and MoH. J. Am. Chem. Sot., 1985, 107,4379 .

22. L. Li, J. T. Millay, J.P. Turner, F.L. King, Millisecond Pulsed Radio Frequency Glow Discharge Time of Flight Mass Spectrometry: Temporal and Spatial Variations in Molecular Energetics. Journal of Am. Soc. Mass Spectrom., 2004, 15, $87-102$.

23. http://webbook.nist.gov/chemistry 


\section{Chapter 3}

\section{Direct Iron Oxide $\left(\mathrm{Fe}_{\mathrm{x}} \mathrm{O}_{\mathrm{y}}\right)$ Speciation in Solid State Materials by Pulsed Millisecond Radio Frequency Glow Discharge Time-of-Flight Mass Spectrometry}

\subsection{Introduction}

Speciation of elemental oxidation state is of great interest in analytical chemistry because it impacts the toxicity, metabolism, transport, and refining method for the material. Traditional methods of analysis via atomic or mass spectrometry techniques require solid samples to be dissolved before analysis. ${ }^{1,2}$ Pretreatment procedures, such as extraction, preconcentration and sometimes derivatization, not only increase analysis time and cost but may change elemental oxidation states. ${ }^{3}$ It is clear that direct solid state sample analysis methods that can yield speciation without changing a sample's oxidation state during sampling, pretreatment and measurement provide the best approach in speciation. ${ }^{4}$

$\mathrm{X}$-ray fluorescence spectroscopy is also a traditional technique for elemental analysis of solid samples. Experiments by Deluigi indicate that direct speciation may also be carried out by investigation of energy shift and intensity ratio of certain lines. ${ }^{5}$ Unfortunately line energy, width, or intensity ratios are not specific enough to provide oxide speciation for salts. ${ }^{6}$ Several mass spectrometry based 
methods also provide oxidation state speciation. Aubriet tried to distinguish several first-row transition metal oxides with static secondary ion mass spectrometry (s-SIMS) on the basis of characteristic ions, ion abundance ratios and a valence model. ${ }^{3}$ The same group also investigated Laser ablation/ionization fourier transform ion cyclotron resonance mass spectrometry (LA-FTICRMS) as a technique for chromium speciation. ${ }^{7}$ A key finding from their work in which s-SIMS and LA-FTICRMS experiments are compared, was that the distribution of metal valence states is related to metal electronic shell configuration and the oxygen to metal ratio in oxides will affect metal atom valence state. ${ }^{8}$ Hachimi reports discrimination of nickel, chromium, and lead oxides via Laser Microprobe Mass Spectrometry (LMMS), another important elemental analysis technique for solids. Application of this method to biological samples was also reported. ${ }^{9}$ In related work, Maunit studied iron oxide cluster formation mechanisms and discriminated between three iron oxide powders by comparing ion abundance ratios obtained from LMMS. ${ }^{10}$ Recently, Hang reported iron oxide speciation accomplished using laser ionization orthogonal time-of-flight mass spectrometry (LI-O-TOFMS). In his work, stable ion abundance ratios were used to discriminate iron oxides and elucidate the mechanism of cluster formation. ${ }^{6}$

Glow discharge mass spectrometry has been used for direct trace elemental analysis of solid samples for more than 30 years. ${ }^{11}$ The introduction of radio frequency glow discharge instruments extended the technique's applicability to non-conductive samples. ${ }^{12}$ Pulsed power operation has been demonstrated to 
provide higher ionization efficiency while preventing sample overheating. ${ }^{13}$ More importantly, this approach allows different ionization processes to be separated by temporal selection within three distinct time regimes (prepeak, plateau and afterpeak) for each cycle. The prepeak regime is dominated by electron ionization (EI); whereas, the plateau regime is a convolution of EI, charge transfer, and Penning ionization (PI) processes. The afterpeak regime is dominated by PI. ${ }^{14}$ By selecting appropriate experimental conditions, the plasma can be tuned to supply specific ionization and thereby specific information for analytes of interest. ${ }^{15}$

Previous work in this lab demonstrated ability of direct speciation of manganese (II, IV) and chromium (III, VI) in solid state samples. ${ }^{4,16}$ Here we extend the pulsed radio frequency glow discharge time-of-flight mass spectrometry method to iron (II) monoxide (FeO), iron (III) oxide $\left(\mathrm{Fe}_{2} \mathrm{O}_{3}\right)$ and iron (II, III) oxide $\left(\mathrm{Fe}_{3} \mathrm{O}_{4}\right)$ speciation in solid state samples. Temporal and spatial settings of GDMS are discussed. Study of glow discharge operating conditions, such as discharge gas pressure, power, and pulse settings, are presented. Discrimination of three iron oxides is achieved by ion abundance ratio comparison at optimized condition.

\subsection{Experimental}

Radio frequency power to the system is supplied by a $13.56 \mathrm{MHz}$ radio frequency (RF) generator equipped with an automatic matching network (RF Plasma Products Inc., Marlton, NJ). Applied power, pulse width, and duty cycle can be controlled by the on-board CPU of the generator. In this project, operating 
power was set at $80 \mathrm{~W}$. The RF pulse width was set initially at $5 \mathrm{~ms}$ with $25 \%$ duty cycle but varied to find optimal conditions for iron oxide speciation. Argon (ultra pure, Airgas, Randor, PA) was introduced into the glow discharge chamber as the discharge support gas through a metering valve and the pressure was monitored via a thermocouple pressure gauge (Varian, Lexington, MA).

Analytical reagent grades of Iron (II) monoxide (FeO), Iron (III) oxide $\left(\mathrm{Fe}_{2} \mathrm{O}_{3}\right)$ and Iron (II, III) oxide $\left(\mathrm{Fe}_{3} \mathrm{O}_{4}\right)$ were purchased from commercial suppliers (Alfa Aesar, Ward Hill, MA and Aldrich, St. Louis, MO). These iron oxides were mixed with spectroscopic grade silver powder (99.999\%, Alfa Aesar, Ward Hill, MA) at different concentrations by weighing. The mixed powders were homogenized for 3 min using a laboratory mixer (Wig-L-Bug, SPEX, Edison, NJ). Approximately $0.6 \mathrm{~g}$ of powder was then compacted in a die assembly constructed in the department workshop under 4000 psi for 30 min to yield sample disks $5 \mathrm{~mm}$ in diameter and $\sim 6 \mathrm{~mm}$ in height. Both iron oxide powder and pressed pellets are baked overnight at $110{ }^{\circ} \mathrm{C}$ before use. Sample disks were introduced on the tip of the direct insertion probe (DIP) and served as the glow discharge cathode. The distance between the sample cathode and ion sampling orifice anode plate was adjusted by moving DIP axially. Cathode samples were shielded by a non-conductive $\mathrm{MACOR}^{\circledR}$ shield (Accuratus, Washington, N.J.) to maintain cathode surface area and current density while restricting sputtering to sample top surface. The Time-of-Flight Mass Spectrometry operating settings are listed in Table 3.1 and further information can be found in previous papers regarding this 
system. ${ }^{4,16}$

Table 3.1 Pulsed RF GD-TOFMS operating parameters

\section{Glow discharge}

Pressure

0.2-0.6 Torr

Operating power

$50-110 \mathrm{~W}$

Pulse duration

$1-5 \mathrm{~ms}$

Duty cycle

$10-50 \%$

Sampling distance

5-20 mm

Time-of-flight

Flight path length

$1 \mathrm{~m}$

Ion optic potentials

Skimmer $-350 \mathrm{~V}$

Accelerator(A2)

Deflector (X1)

$-1800 \mathrm{~V}$

Deflector (Y1)

$-1495 \mathrm{~V}$

Repeller(A1)

Detector

\subsection{Results and Discussion}

\subsubsection{Optimization of Temporal Regimes}

As shown in Figure 3.1, RF power is applied in a repeating square wave pulse.

As a result, glow discharge plasma exhibits three distinct temporal regimes: prepeak, plateau, and afterpeak. Spectra from these regimes were compared for pure silver pellets and pellets with 25\% FexOy and 75\% silver in weight. For temporal evaluation studies, Ar pressure is set at 0.4 torr, RF power is set at $80 \mathrm{~W}$, and the distance between cathode surface and anode is fixed at $10 \mathrm{~mm}$. 

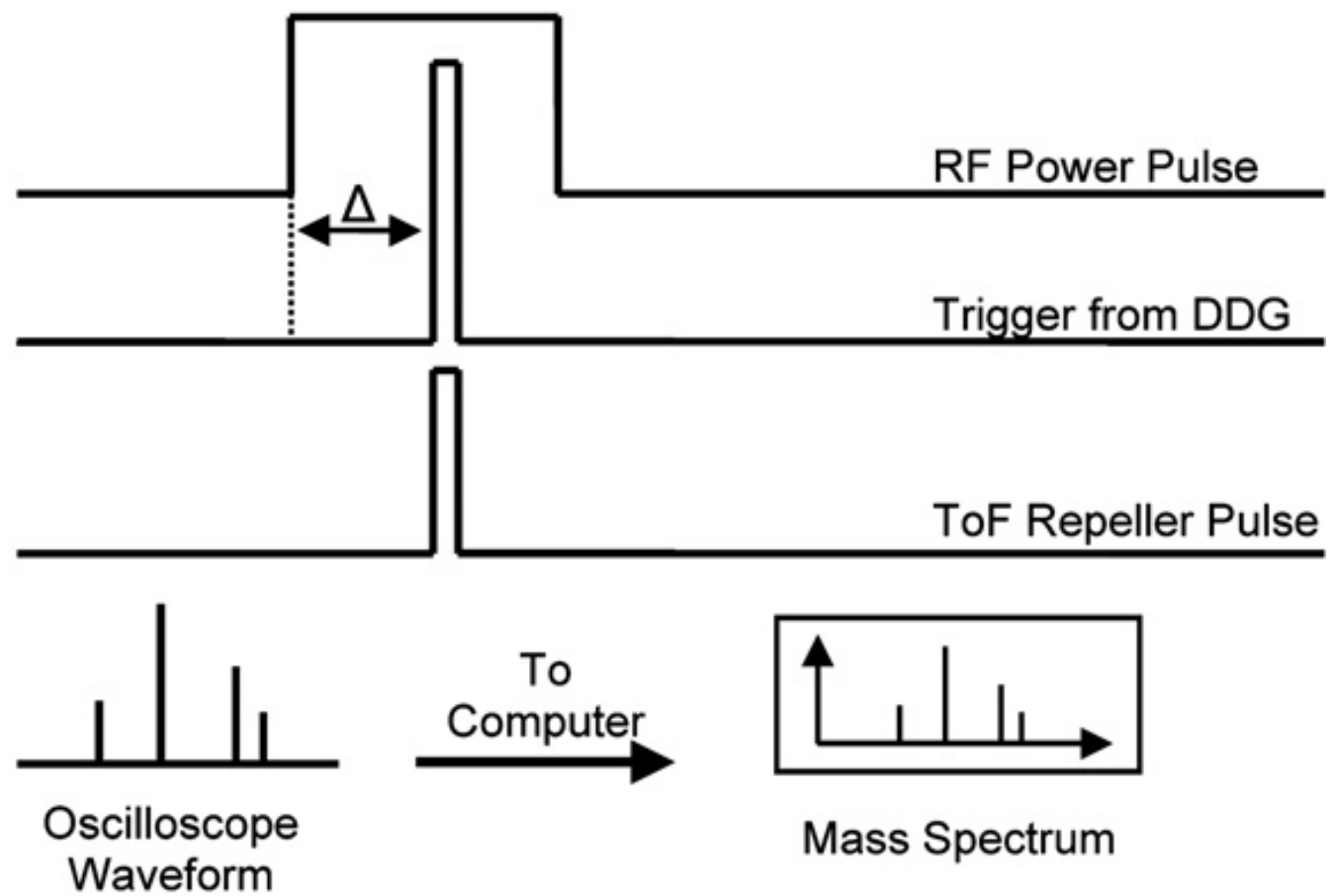

Figure 3.1 Data acquisition of RF GD-TOFMS. The digital delay generator (DDG) provides a trigger delayed by a variable time $(\Delta)$ from the initiation of the RF power pulse. This delayed trigger initiates data acquisition by the digital storage oscilloscope (DSO) and the repeller pulse that injects ions into the Time of Flight mass spectrometer. Ion arrival at the micro-channel plate detector is monitored with the DSO and converted to a mass spectrum in EXCEL.

Prepeak. The prepeak regime endures for several hundred micro-seconds after initiation of the power pulse. The applied potential accelerates electrons from the cathode toward the anode. Ionizing collisions between these accelerated electrons and discharge gas molecules (Ar) produce discharge gas ions $\left(\mathrm{Ar}^{+}\right)$and secondary electrons. Sputtering of the sample cathode commences as the population of argon 
ions increases and accelerates to the cathode surface. The mass spectrum reflects this as it contains signals only for species present in the discharge gas, such as Ar, air and water. Figure 3.2 shows mass spectra collected $0.3 \mathrm{~ms}$ after power-on for pure silver pellet and pellet with $25 \% \mathrm{FeO}$ and $75 \%$ silver w/w. As mentioned above, spectra are mainly composed of gaseous ions such as $\mathrm{Ar}^{+}, \mathrm{Ar}^{+2}$, and $\mathrm{H}_{2} \mathrm{O}^{+}$ ( $\mathrm{m} / \mathrm{z} 40,20,18$ respectively) and no cathode material is detected. Therefore, spectra of pure silver and FexOy/ mixture pellets are all very similar in this time regime, no matter the FexOy identity. 


\section{(a) Pure silver}

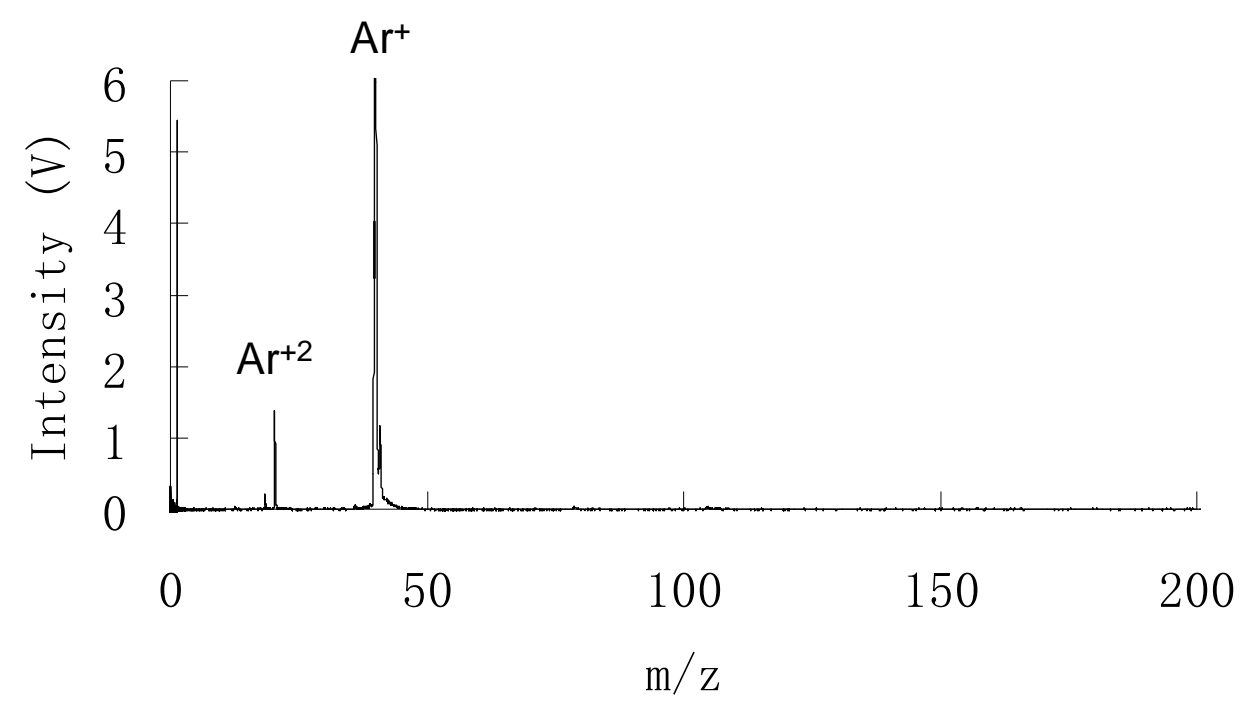

(b) $25 \% \mathrm{FeO}+\mathrm{Ag}$

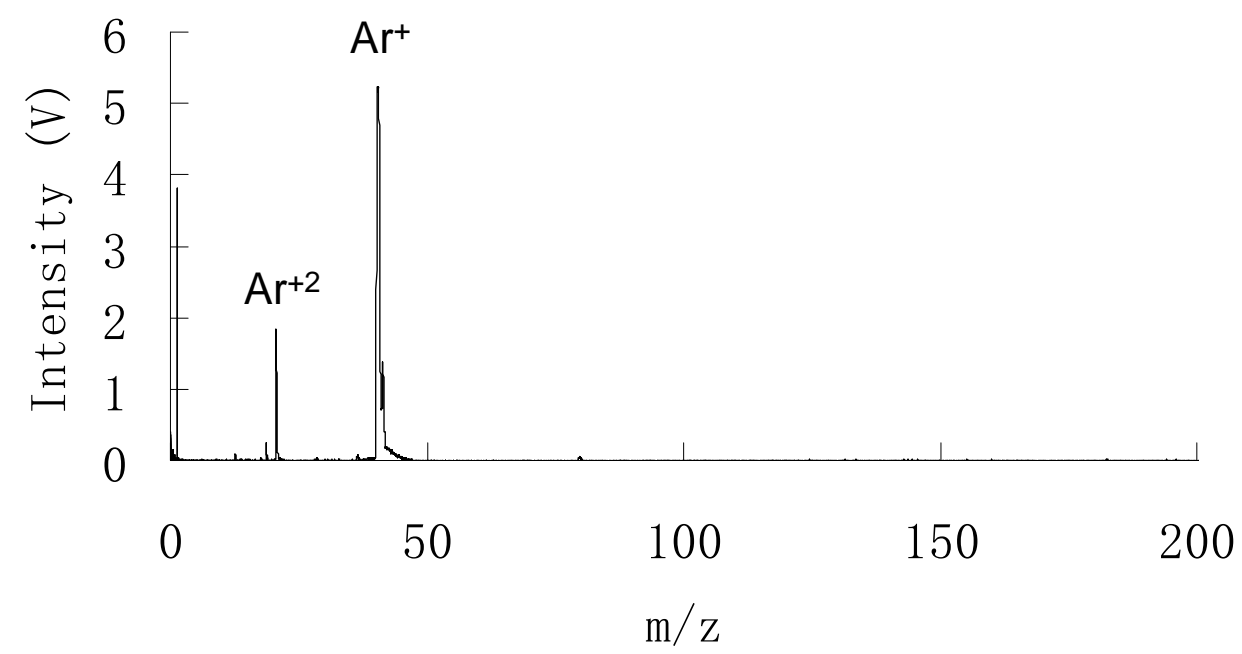

Figure 3.2 Time resolved mass spectra of (a) pure $\mathrm{Ag}$ and (b) 25\% FeO/Ag samples during the prepeak (0.3 ms after power-on). Discharge pressure 0.4 Torr, operating power $80 \mathrm{~W}$, pulse duration $5 \mathrm{~ms}$ with $25 \%$ duty cycle and sampling distance $10 \mathrm{~mm}$. 
Plateau. It takes about $1 \mathrm{~ms}$ for plasma to reach a steady state after power application. At this point, electron impact, charge transfer, and Penning ionization processes all exist in an equilibrium state that remains until the power is removed. This period is analogous to that of the steady state glow discharge. As shown in Figure 3.3, both atomic ions and molecular fragments from cathodic material and discharge gases are generated. In spectra from the $\mathrm{Fe}_{\mathrm{x}} \mathrm{O}_{\mathrm{y}}$ and $\mathrm{Ag}$ mixtures, $\mathrm{Fe}^{+}$and weak $\mathrm{Fe}_{\mathrm{x}} \mathrm{O}_{\mathrm{y}}$ cluster ions $\left(\mathrm{FeO}^{+}\right)$appear. Unfortunately, no ions characteristic of the different iron oxides are observed - they all exhibit similar mass spectra.

Afterpeak. After power termination, the fast electron population is rapidly thermalized. The resultant slow electrons recombine with argon ions producing metastable neutral argon atoms. During the afterpeak regime, these metastable argon atoms become the dominant ionization agents, providing for Penning ionization of other species in the plasma. Because this is a soft ionization process, intact molecular ions may be detected as well as atomic ions and molecular fragment ions. Generally, this regime supplies the most significant analytical information for oxidation state determination.

As mentioned in Zhang's paper, ${ }^{4}$ cathode materials have strongest ion intensities at $0.3 \mathrm{~ms}$ after power-off. Figure 3.4 shows mass spectra of pellets with $25 \% \mathrm{Fe}_{\mathrm{x}} \mathrm{O}_{\mathrm{y}}$ collected at $5.3 \mathrm{~ms}$. Compared with Figure 3.3, Ag peak intensities in those spectra increase significantly and $\mathrm{Ag}-\mathrm{H}_{2} \mathrm{O}$ clusters may also be observed. For $\mathrm{Fe}_{\mathrm{x}} \mathrm{O}_{\mathrm{y}}$ containing pellets, atomic ions and cluster ions like $\mathrm{Fe}^{+}, \mathrm{FeO}^{+}, \mathrm{FeOH}^{+}$and 
$\mathrm{FeOH}_{3}{ }^{+}(\mathrm{m} / \mathrm{z}$ 56, 72, 73 and 75 respectively) appear. In agreement with Maunit etc.'s work, ${ }^{10}$ no ions characteristic of each oxidation state are found. However, the ratio between these ion's intensities was found to permit $\mathrm{Fe}_{\mathrm{x}} \mathrm{O}_{\mathrm{y}}$ speciation as discussed below.

\subsubsection{Optimization of Sampling Distance}

According to previous experiments, particle spatial distributions in the glow discharge relate to excitation and ionization processes as well as analyte identity. By changing the distance between cathode surface and anodic ion exit orifice, signal can be optimized for the analyte(s) of interest. For the purpose of iron oxide speciation, a pellet with $25 \% \mathrm{Fe}_{3} \mathrm{O}_{4}$ and $75 \% \mathrm{Ag}$ in weight serves as the cathode in a plasma operating at $80 \mathrm{~W}$ and 0.4 Torr Ar during the afterpeak. As the distance between the surface of cathode and anode was varied from 5 to $20 \mathrm{~mm}$, it was found that at distances shorter than $5 \mathrm{~mm}$, no stable spectrum could be obtained. In addition it was noted that cluster ion intensities become too low to be recognized at distances greater than $20 \mathrm{~mm}$. 


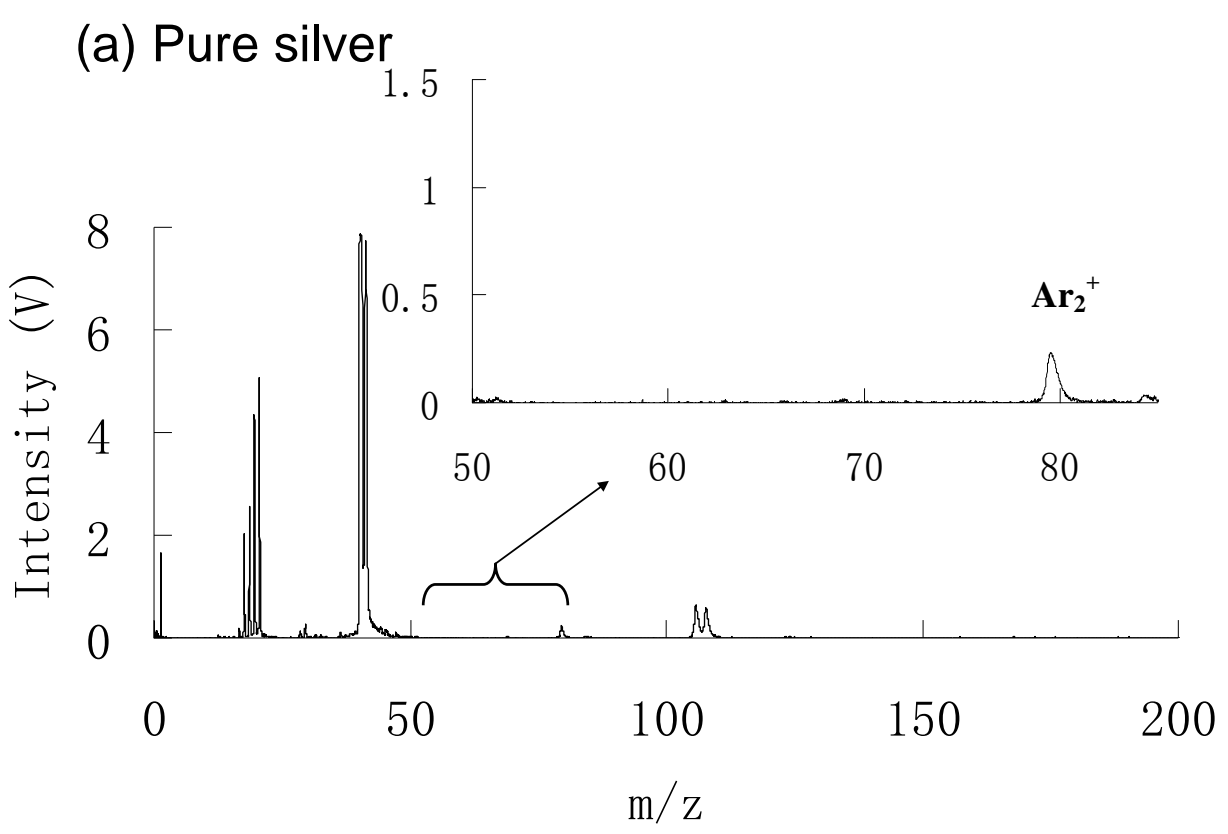

(b) $25 \% \mathrm{FeO}+\mathrm{Ag}$

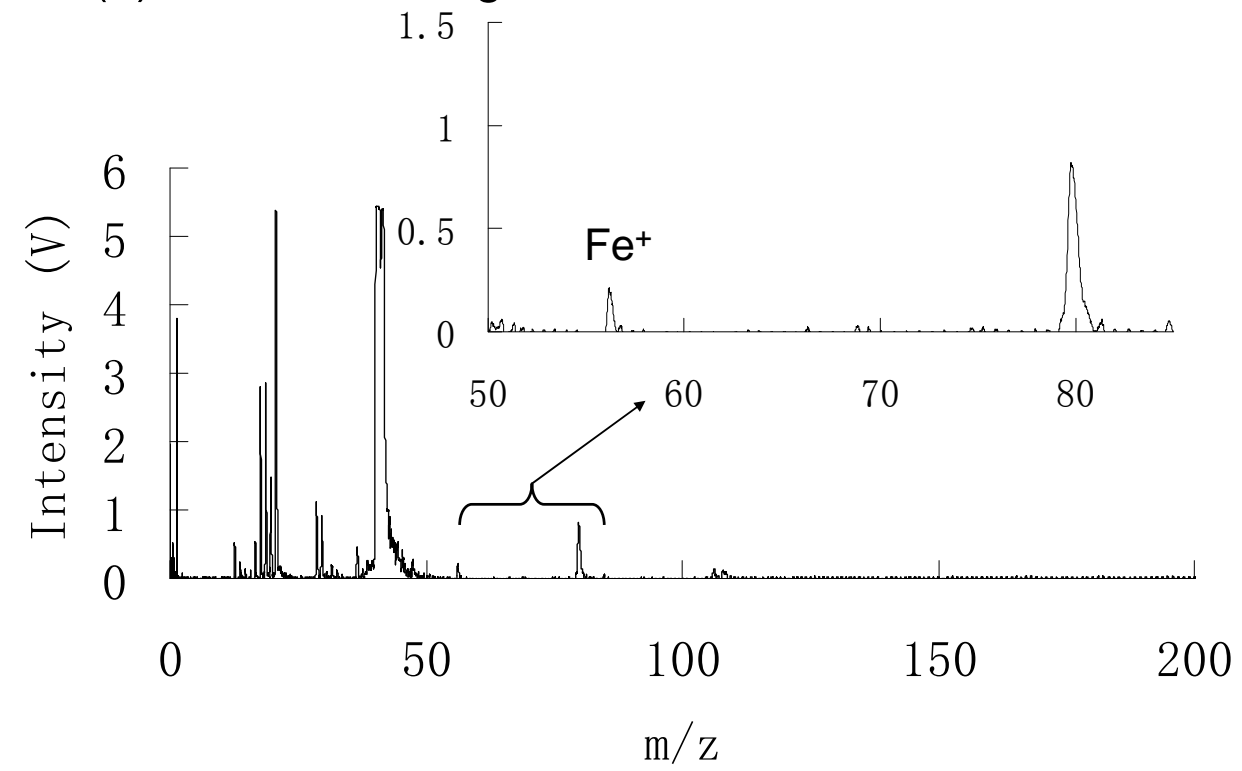


(c) $25 \% \mathrm{Fe}_{2} \mathrm{O}_{3}+\mathrm{Ag}$

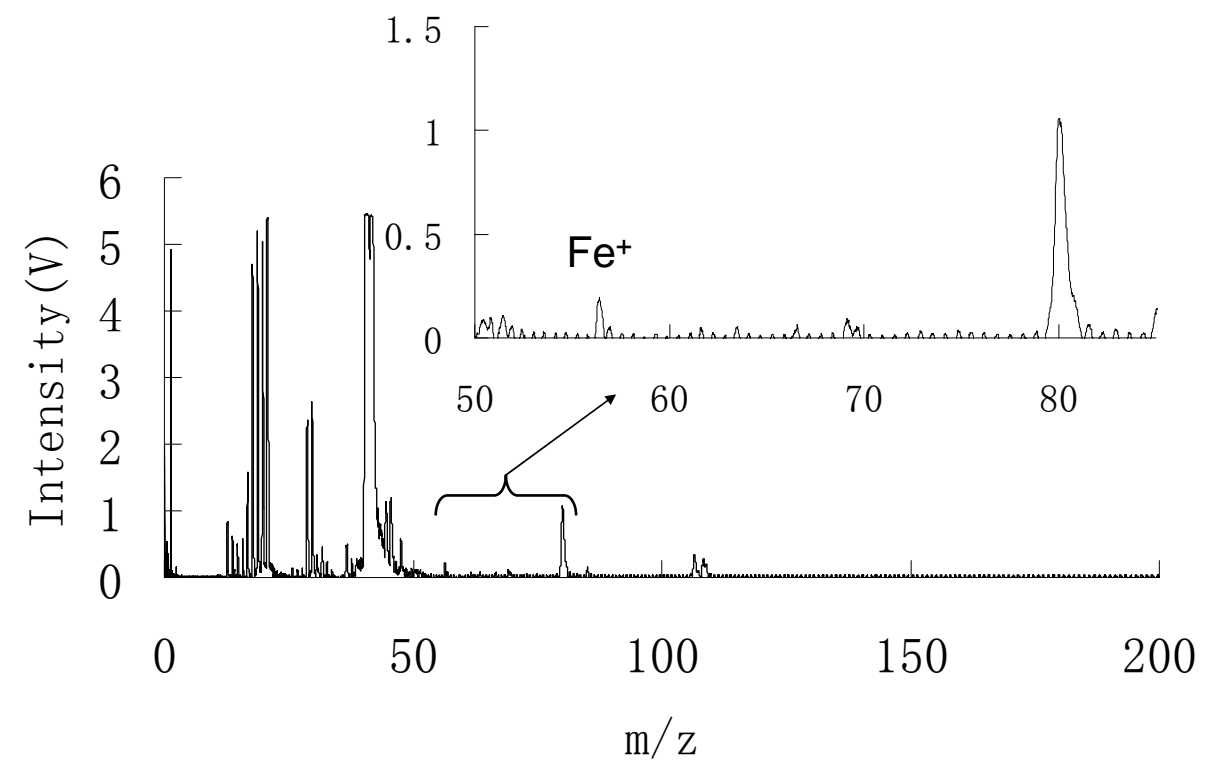

(d) $25 \% \mathrm{Fe}_{3} \mathrm{O}_{4}+\mathrm{Ag}$

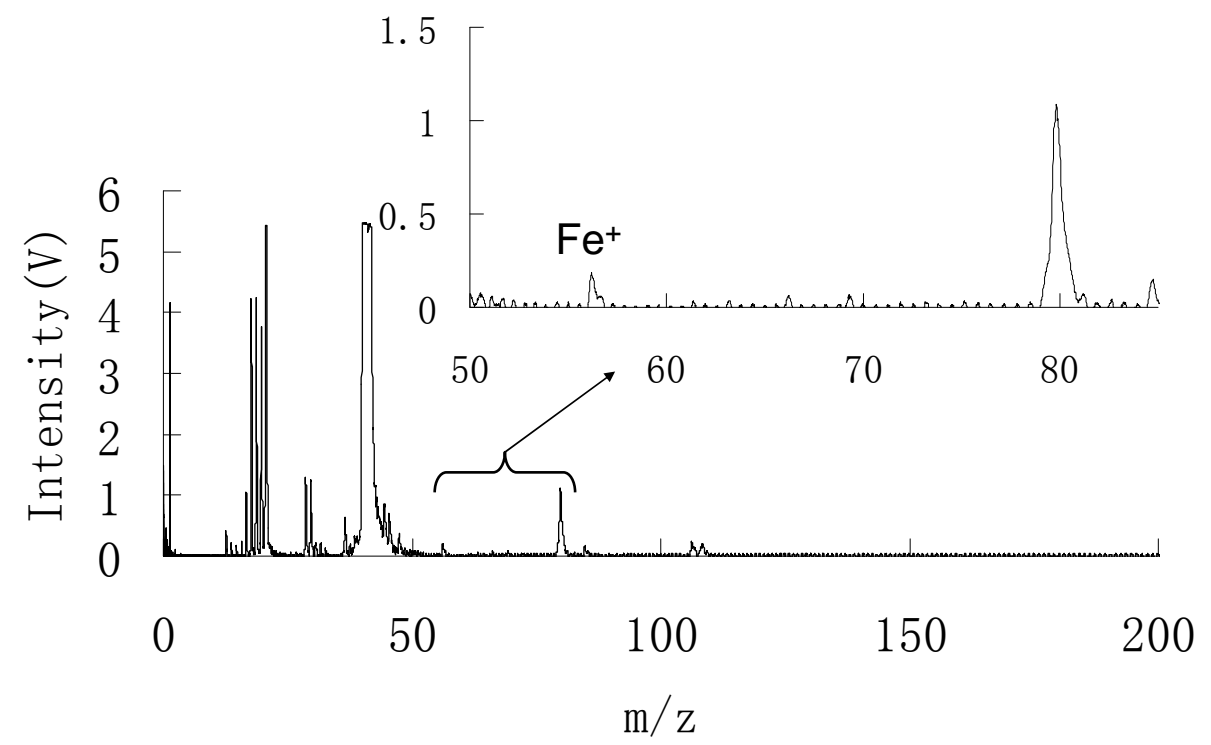

Figure 3.3 Time resolved mass spectra of (a) pure $\mathrm{Ag}$ and (b) $25 \% \mathrm{FeO} / \mathrm{Ag}$, (c) $25 \%$ $\mathrm{Fe}_{2} \mathrm{O}_{3} / \mathrm{Ag}$, and (d) $25 \% \mathrm{Fe}_{3} \mathrm{O}_{4} / \mathrm{Ag}$ during the plateau (1.3 $\mathrm{ms}$ after power-on). Discharge pressure 0.4 Torr, operating power $80 \mathrm{~W}$, pulse duration $5 \mathrm{~ms}$ with $25 \%$ duty cycle and sampling distance $10 \mathrm{~mm}$. 
(a) Pure silver

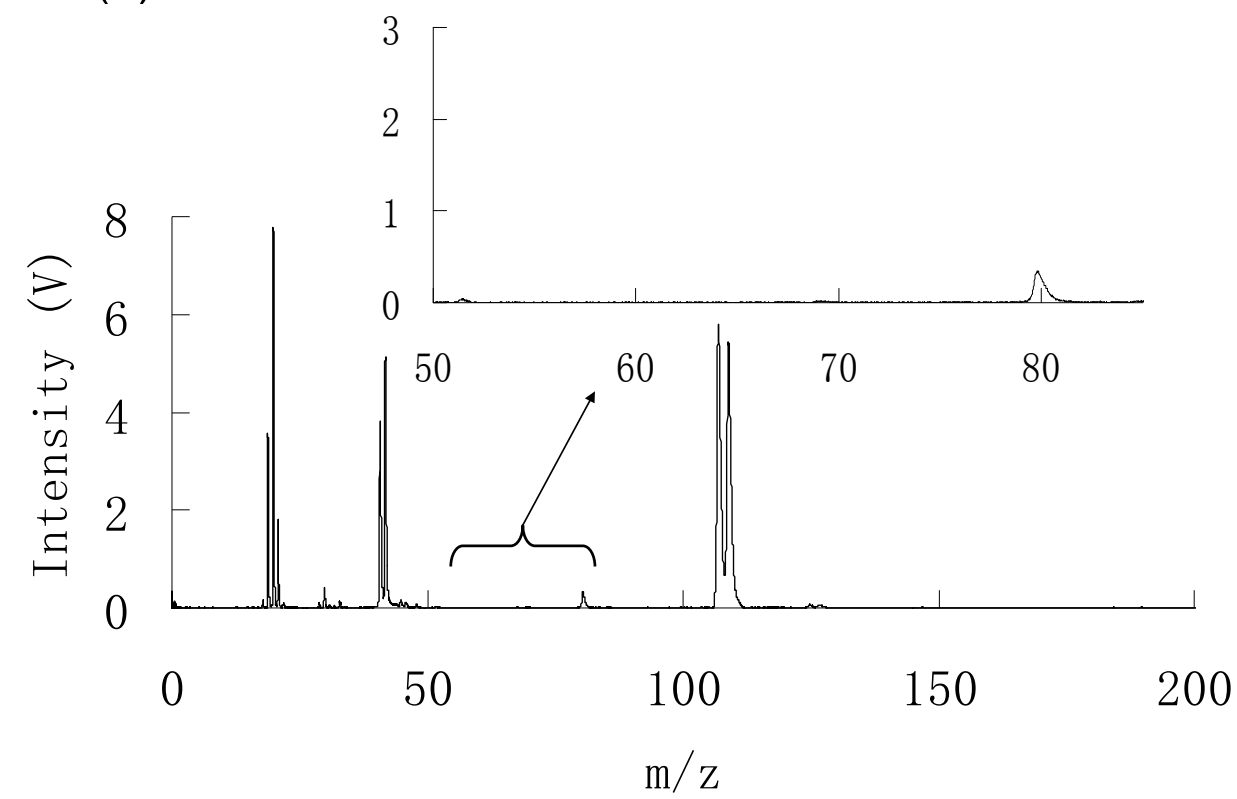

(b) $25 \% \mathrm{FeO}+\mathrm{Ag}$

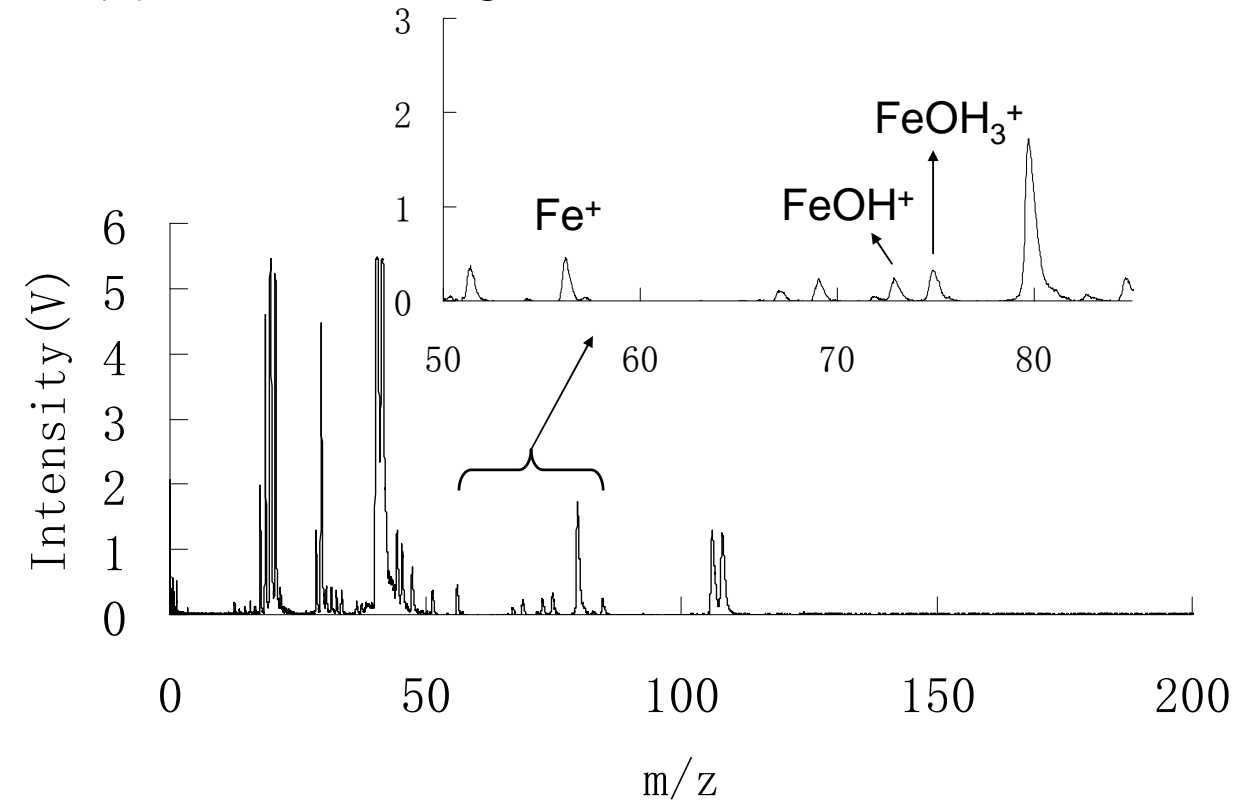


(c) $25 \% \mathrm{Fe}_{2} \mathrm{O}_{3}+\mathrm{Ag}$

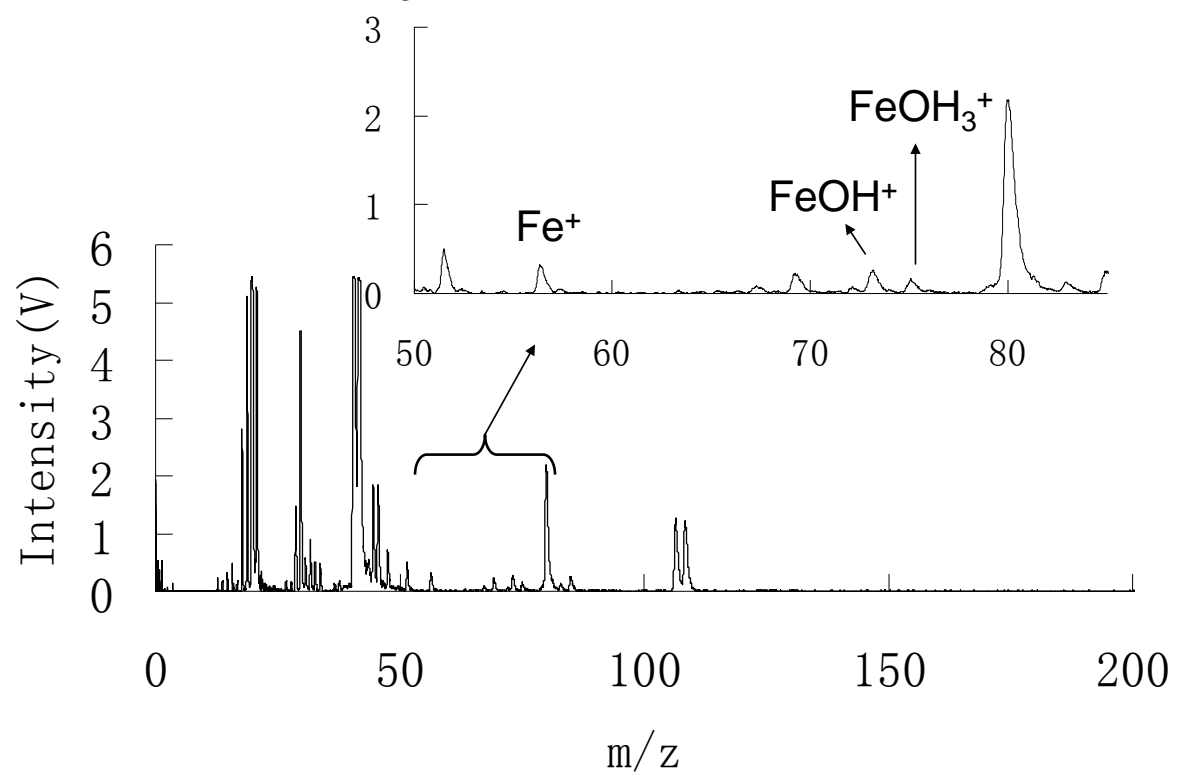

(d) $25 \% \mathrm{Fe}_{3} \mathrm{O}_{4}+\mathrm{Ag}$

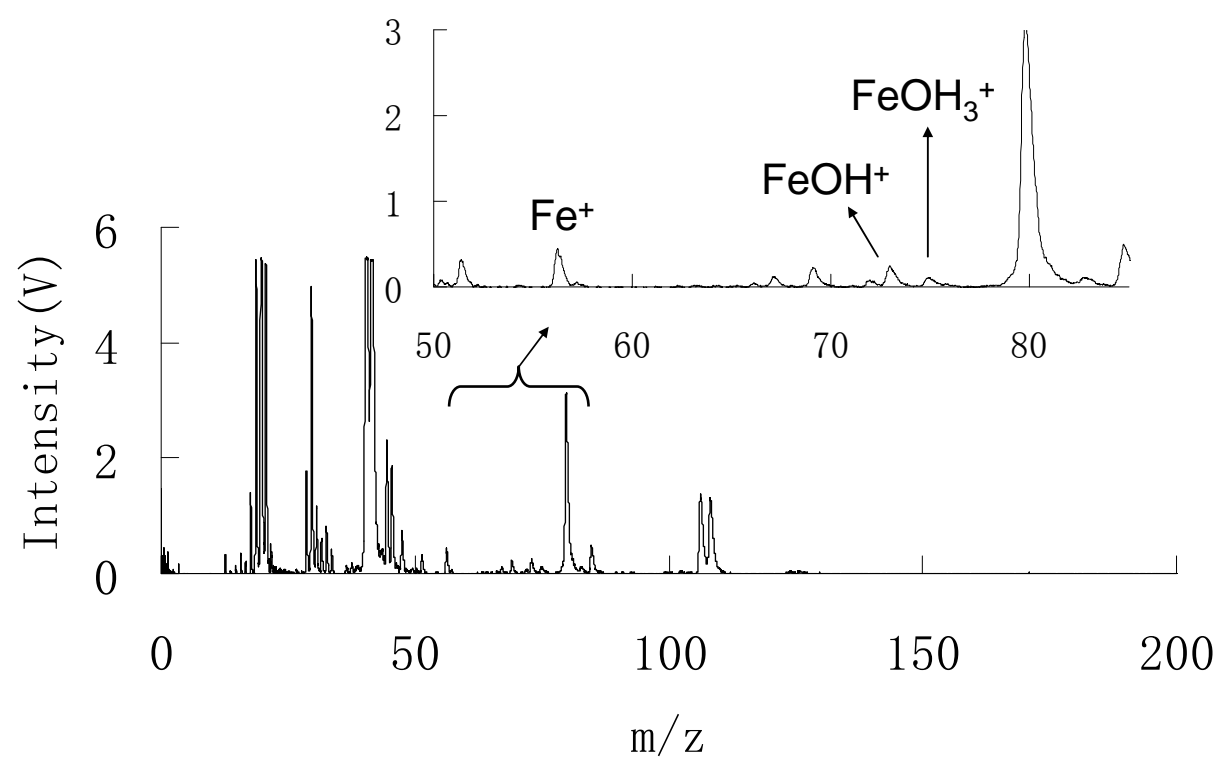

Figure 3.4 Time resolved mass spectra of (a) pure $\mathrm{Ag}$ (b) $25 \% \mathrm{FeO} / \mathrm{Ag}$, (c) $25 \%$ $\mathrm{Fe}_{2} \mathrm{O}_{3} / \mathrm{Ag}$, and (d) $25 \% \mathrm{Fe}_{3} \mathrm{O}_{4} / \mathrm{Ag}$ samples during the afterpeak $(5.3 \mathrm{~ms}$ after power-on, $1.3 \mathrm{~ms}$ after power-off). Discharge pressure 0.4 Torr, operating power $80 \mathrm{~W}$, pulse duration $5 \mathrm{~ms}$ with $25 \%$ duty cycle and sampling distance $10 \mathrm{~mm}$. 


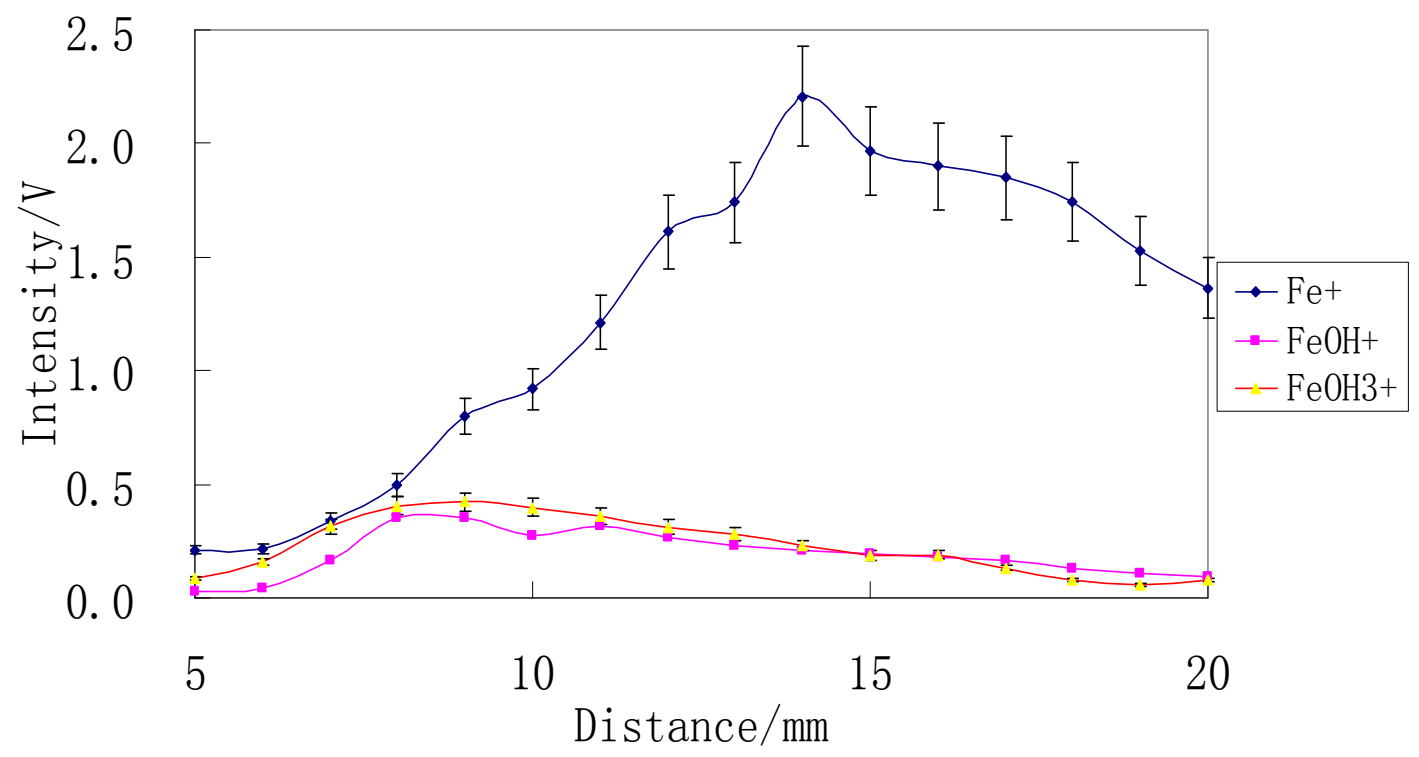

Figure 3.5 Spatial effect on ion signals for representative $\mathrm{Fe}_{3} \mathrm{O}_{4}: \mathrm{Ag}=25: 75$ (m:m) sample pellet. The glow discharge power is $80 \mathrm{w}$, and the discharge gas pressure is 0.4 torr. Each data point is the result of 3 measurements and error bars represent one standard deviation from the mean value.

The maximum intensities for $\mathrm{Fe}^{+}(\mathrm{m} / \mathrm{z} 56), \mathrm{FeOH}^{+}(\mathrm{m} / \mathrm{z} 73)$ and $\mathrm{FeOH}_{3}{ }^{+}(\mathrm{m} / \mathrm{z}$ 75) ions versus distance are plotted in Figure $3.5 . \mathrm{Fe}^{+}(\mathrm{m} / \mathrm{z} 56)$ ion intensity increases as distance increases and maximizes at $\sim 14 \mathrm{~mm}$ and then decreases at greater distances. $\mathrm{FeOH}^{+}$and $\mathrm{FeOH}_{3}{ }^{+}$both exhibit trends similar to one another with respect to spatial signal optimization. Their intensities increase when the distance change from 5 to $8 \mathrm{~mm}$ and reach maximum value at about 8 to $9 \mathrm{~mm}$, where both metastable argon atom and Penning ionization processes were found to maximize in previous work. ${ }^{19,20}$ After that, ions intensities begin to decrease as the 
distance increases from 9 to $20 \mathrm{~mm}$. These spatial results indicate that cluster ions such as $\mathrm{FeOH}^{+}$and $\mathrm{FeOH}_{3}{ }^{+}$likely result from precursors dependent on Penning ionization during afterpeak period; whereas, $\mathrm{Fe}^{+}$arises from both direct Penning ionization of $\mathrm{Fe}$ and cluster ion dissociation.

\subsubsection{Optimization of Glow Discharge Operating Parameters}

Excitation and ionization processes in glow discharges are affected by parameters such as operating power and discharge gas pressure. Those parameters were systematically evaluated and adjusted to optimize the signals for $\mathrm{m} / \mathrm{z} 73$ and 75 ions.

\subsubsection{Operating Power}

The power effect was examined by changing RF operating power from 50 to $110 \mathrm{~W}$, while the distance between the surface of pellet and anode fixed at $10 \mathrm{~mm}$ and the discharge gas pressure remains at 0.4 Torr. The pellet used in these test has $25 \% \mathrm{Fe}_{3} \mathrm{O}_{4}$ and $75 \% \mathrm{Ag}$ in weight. Based on previous work in our lab, the power change will affect signal intensity significantly, but it will not affect energy distribution. ${ }^{4,16}$ Figure 3.6 shows the maximum intensity of $\mathrm{m} / \mathrm{z} 56,73$ and 75 ions during afterpeak regime as a function of operating power. The intensity of $\mathrm{m} / \mathrm{z}$ 56 increases as the power increases from 50 to $90 \mathrm{~W}$. This can be explained by the increase of sputter rate and ionization that accompanies increases in operating power. Although the data presented in Figure 3.6 indicate that the optimal 
operating power for iron oxide speciation experiments is $90 \mathrm{~W}$, a lower setting of $80 \mathrm{~W}$ was used in subsequent experiments so that they could be compared with previous speciation work done in this lab at $80 \mathrm{~W}$.

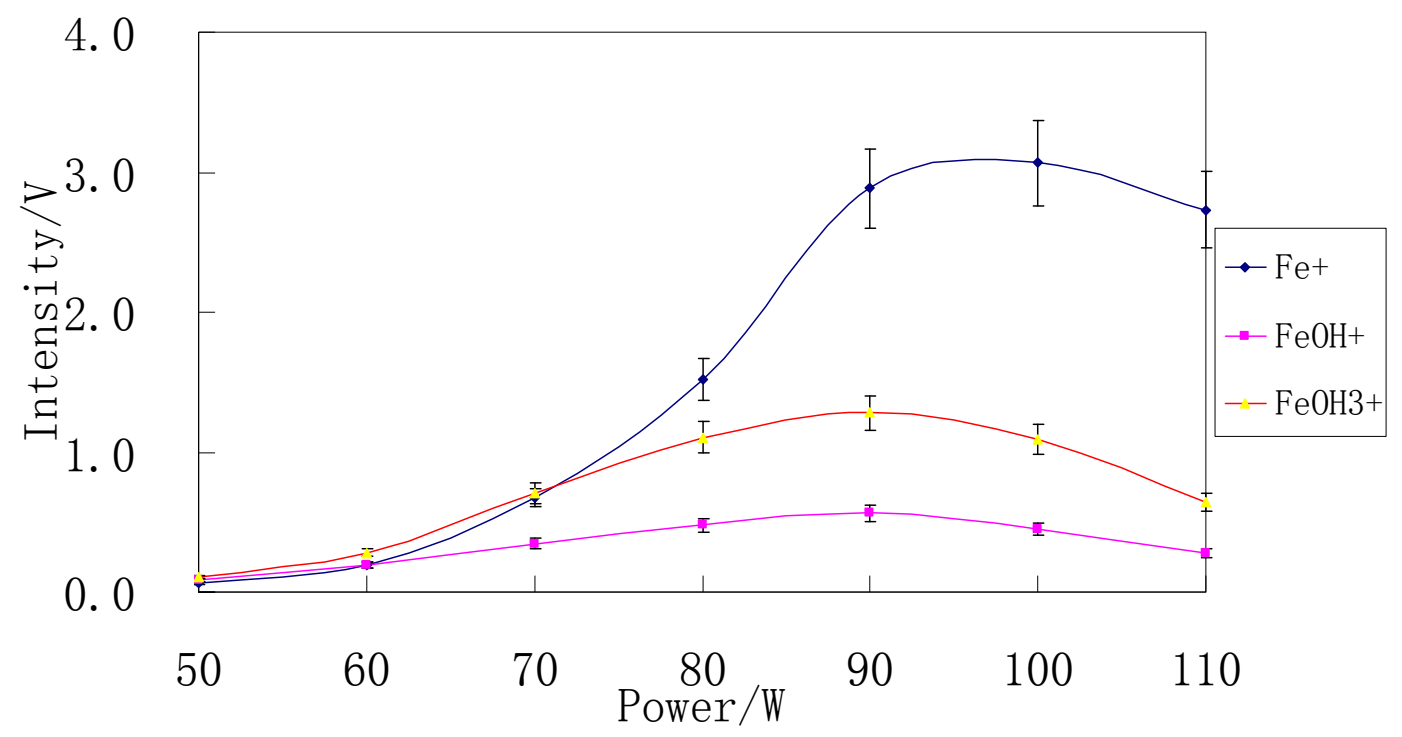

Figure 3.6 Power effect on ion signals for $\mathrm{Fe}_{3} \mathrm{O}_{4}: \mathrm{Ag}=25: 75$ (m:m) sample pellet. The glow discharge pressure is 0.4 torr Ar and the sampling distance is $10 \mathrm{~mm}$. Each data point is the result of 3 measurements and error bars represent one standard deviation from the mean value.

\subsubsection{Discharge Gas Pressure}

The discharge gas pressure is related to mean free path and collision frequency of particles in the GD chamber. Figure 3.7 shows the result of 
experiments examining ion intensities as a function of discharge gas pressure. No data are available at pressures lower than 0.2 Torr because of plasma instabilities in that pressure regime. As the discharge gas pressure increases from 0.2 to 0.6 Torr, the intensity of $\mathrm{m} / \mathrm{z} 56$ ion increases, but it appears to increase more slowly at low pressure than at high pressure.

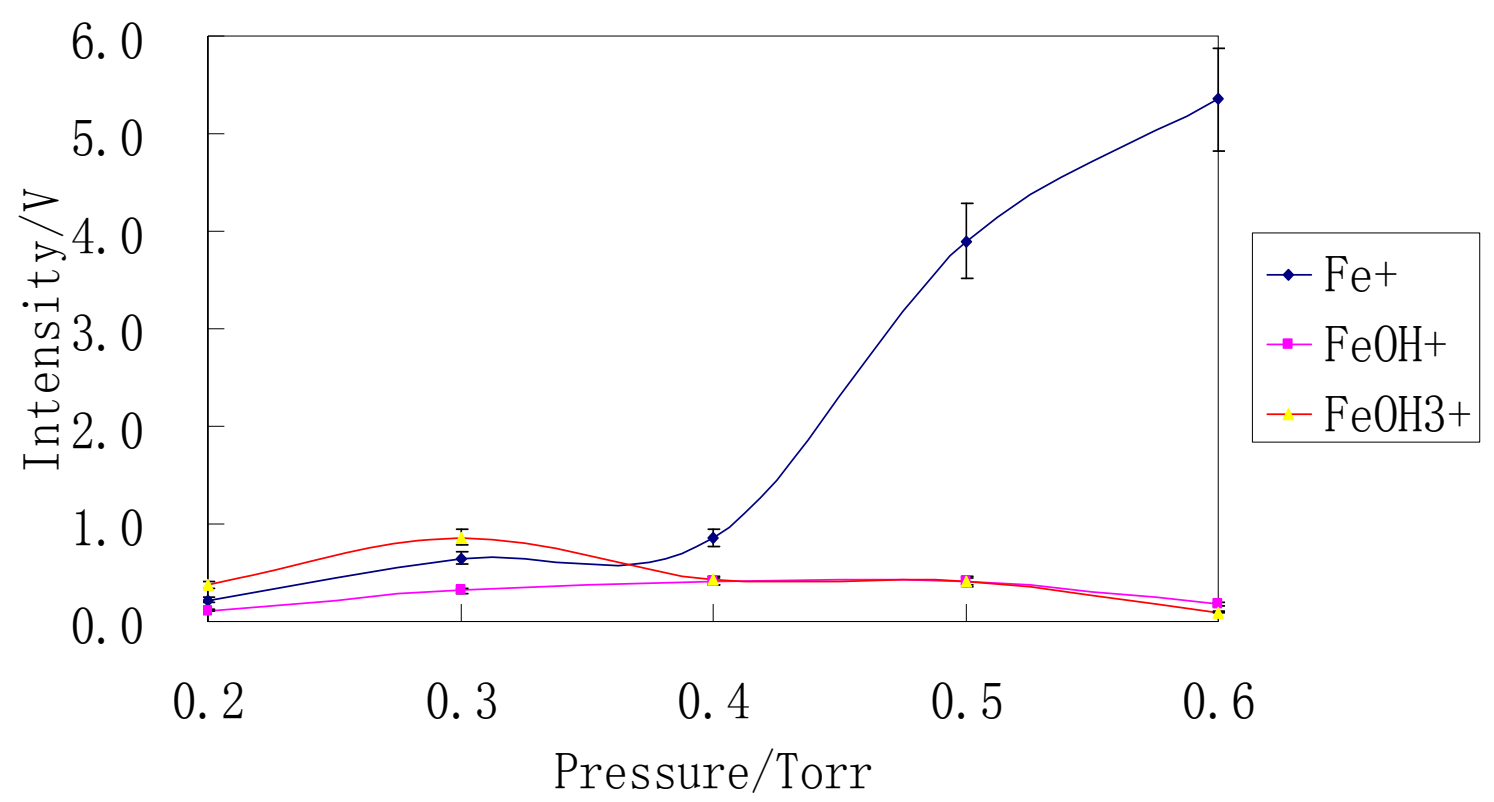

Figure 3.7 Pressure effect on ion signals for $\mathrm{Fe}_{3} \mathrm{O}_{4}: \mathrm{Ag}=25: 75(\mathrm{~m}: \mathrm{m})$ sample pellet. The glow discharge power is $80 \mathrm{~W}$ and the sampling distance is $10 \mathrm{~mm}$. Each data point is the result of 3 measurements and error bars represent one standard deviation from the mean value.

The intensities of $\mathrm{m} / \mathrm{z} 73$ and 75 ions increase from 0.2 Torr to 0.3 Torr, and then decrease as the pressure increases - consistent with increasing collisional dissociation of clusters with increasing pressure. In general, higher pressure operation provides greater elemental signal intensity; whereas, and lower pressure 
operation allows for the persistence of cluster signals. This observation agrees well with experiments performed before in our lab. ${ }^{4,16,17}$ Based on Figure 3.7, the optimal discharge pressure for optimal cluster ion signal intensity in iron oxide speciation experiments appears to be 0.3 Torr.

\subsubsection{Power Pulse Width and Duty Cycle}

The power pulse settings include two parts: pulse width and duty cycle. Experiments are performed separately for these two parts. Figure 3.8 shows the maximum intensity of $\mathrm{m} / \mathrm{z} 56,73$ and 75 ions during afterpeak regime versus different pulse width at $25 \%$ duty cycle. Although peak intensities increase as the pulse width changes from $1 \mathrm{~ms}$ to $2 \mathrm{~ms}$, there is no obvious change after that. As mentioned above, it takes about $1 \mathrm{~ms}$ for plasma to reach a steady state. In the $1 \mathrm{~ms}$, $25 \%$ experiment, the plasma may have not achieved steady state operation, hence the lower ion intensity compared with others. There was no significant effect for pulse widths from $2-5 \mathrm{~ms}$. 


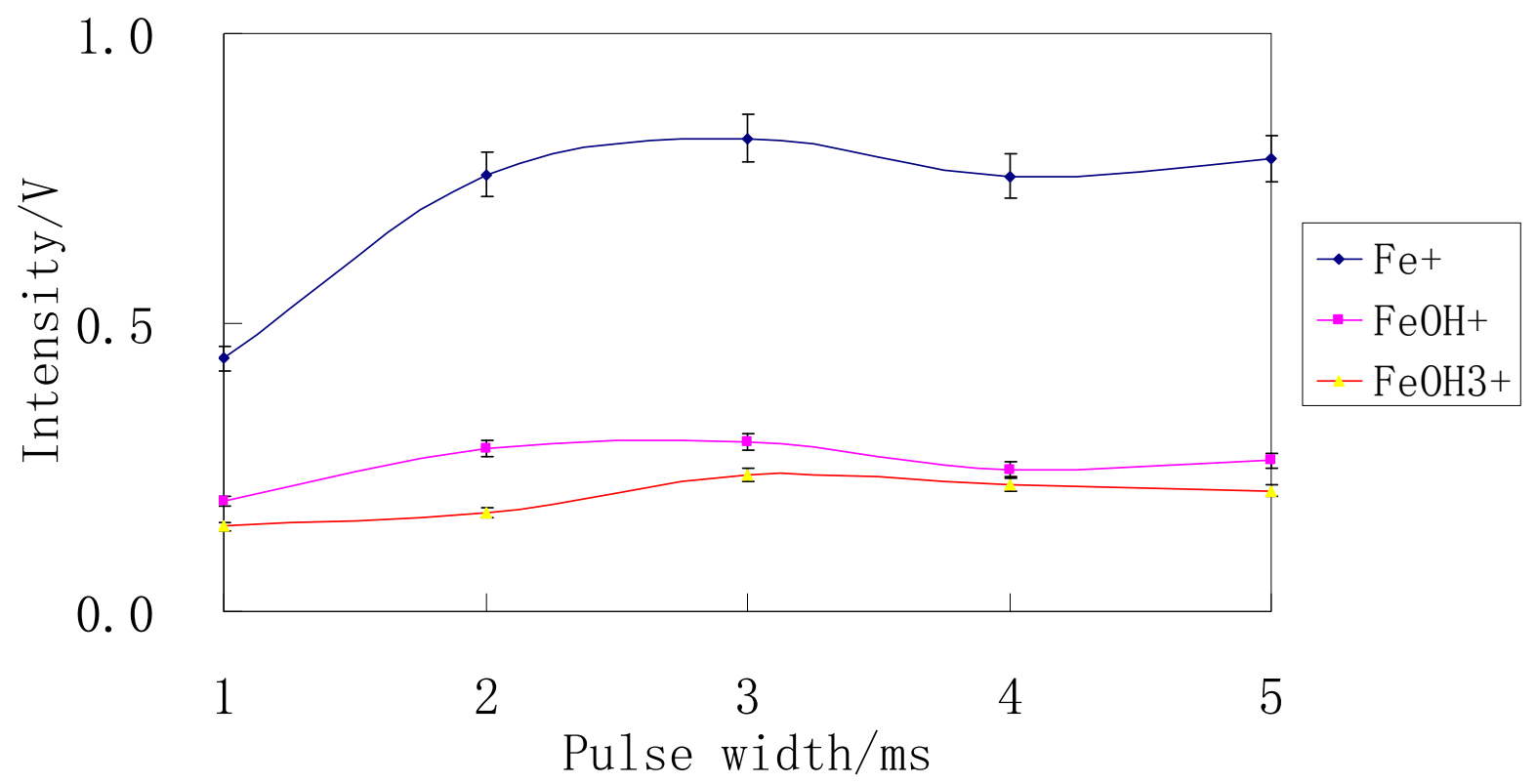

Figure 3.8 Pulse width effect on ion signals at $25 \%$ duty cycle for $\mathrm{Fe}_{3} \mathrm{O}_{4}: \mathrm{Ag}=25: 75$ (m:m) sample pellet. The glow discharge power is $80 \mathrm{~W}$, the discharge gas pressure is 0.4 torr Ar, and the sampling distance is $10 \mathrm{~mm}$. Each data point is the result of 3 measurements and error bars represent one standard deviation from the mean value.

Figure 3.9 shows the maximum intensity of $\mathrm{m} / \mathrm{z} 56,73$ and 75 ions during afterpeak regime against different duty cycle at $2 \mathrm{~ms}$ pulse width. It is observed that the intensity of all three ions decreases slightly as the duty cycle increases from $10 \%$ to $20 \%$. After that, $\mathrm{Fe}^{+}$signal is relatively stable as duty cycle changes from $20 \%$ to $50 \%$. The $\mathrm{FeOH}_{3}{ }^{+}$at $\mathrm{m} / \mathrm{z} 75$ exhibits a trend similar to that for $\mathrm{Fe}^{+}$ except for a slight decrease when duty cycle increased from $40 \%$ to $50 \%$. $\mathrm{FeOH}^{+}$ ion intensity, however, decreases continuously with increasing duty cycle. 


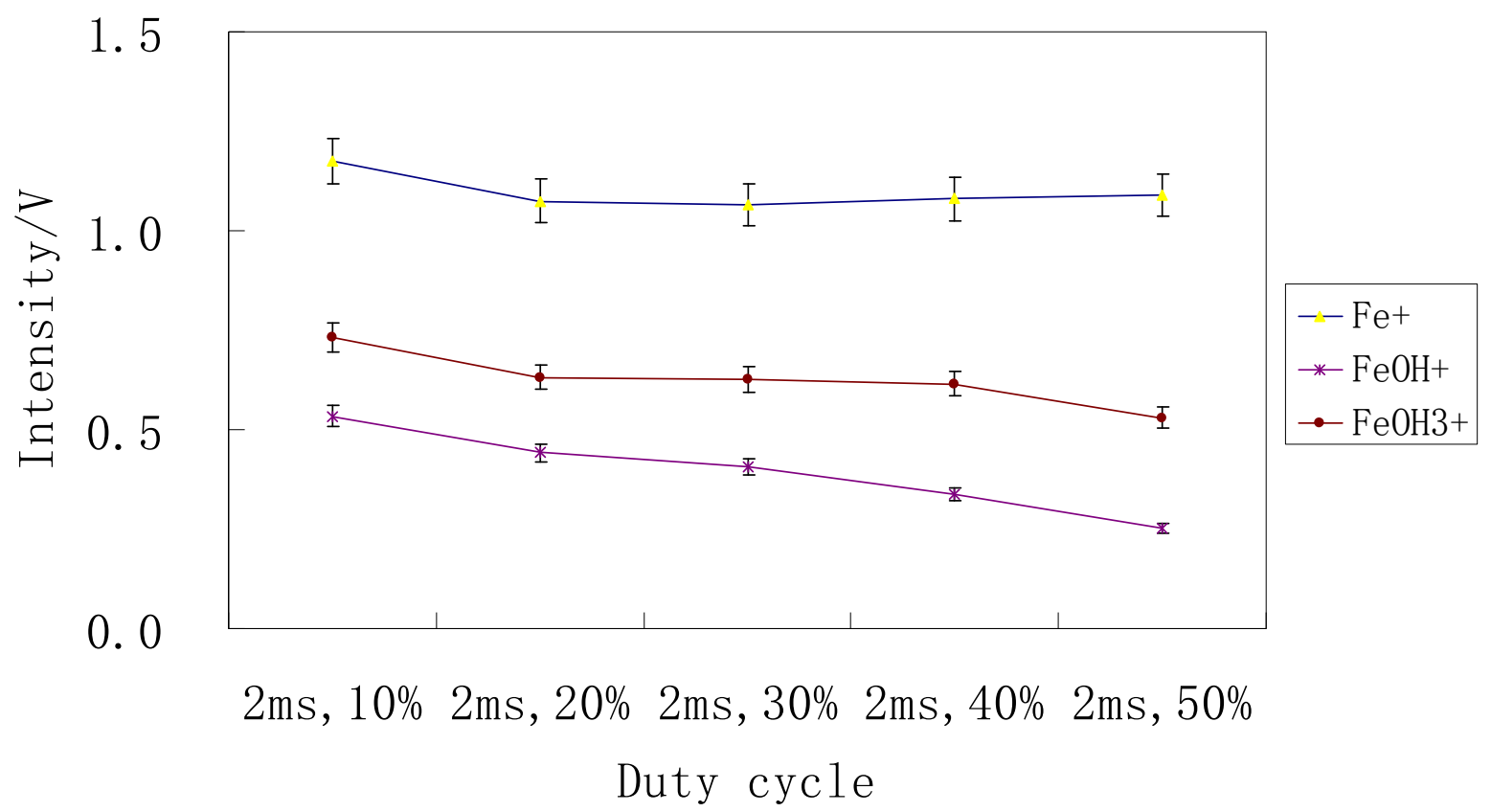

Figure 3.9 Duty cycle effect studied with $\mathrm{Fe}_{3} \mathrm{O}_{4}: \mathrm{Ag}=25: 75$ (m:m) pellet. The glow discharge power is $80 \mathrm{~W}$, the discharge gas pressure is 0.4 torr Ar, and the sampling distance is $10 \mathrm{~mm}$. Each data point is the result of 3 measurements and error bars represent one standard deviation from the mean value.

\subsection{4 $\mathrm{Fe}_{\mathrm{x}} \mathrm{O}_{\mathrm{y}}$ Speciation}

As noted above, no ions characteristic for different oxidation states were found. Spectra obtained from the three different oxide samples all exhibit the same peaks $-\mathrm{Fe}^{+}, \mathrm{FeO}^{+}, \mathrm{FeOH}^{+}$and $\mathrm{FeOH}_{3}^{+}(\mathrm{m} / \mathrm{z}$ 56, 72, 73 and 75 respectively). Previously, Molek observed that $(\mathrm{FeO})_{\mathrm{n}}{ }^{+}$are the most stable of the iron oxide cluster ions. ${ }^{18}$ Certainly the spectra in the present work are consistent with that observation. Unlike Molek's work, however, only the smallest ions of these types are observed. This may result from differences in the production of iron 
oxide clusters. Molek used pure iron metal and oxygen as reactant to generate iron oxide cluster ions in laser-generated plasma. In Maunit's study, using iron oxide powder to generate clusters, only cluster ions smaller than $\mathrm{m} / \mathrm{z} 300$ are observed. This approach is more similar to our approach using pressed iron oxide powders. Additionally, Molek's clusters are generated in a much cooler collisional environment compared with glow discharge afterpeak regime. This cooler collisional environment should favor the persistence of larger cluster ions whereas the more energetic GD will provide greater fragmentation of large clusters.

The mechanisms leading to the formation of $\mathrm{FeO}^{+}, \mathrm{FeOH}^{+}$and $\mathrm{FeOH}_{3}{ }^{+}$likely differ in the precursor species identity and source. $\mathrm{FeOH}_{3}{ }^{+}$is best thought to form as a complex of $\mathrm{Fe}$ with $\mathrm{H}_{3} \mathrm{O}^{+}$, similar to $\mathrm{AgOH}_{3}{ }^{+}$observed in GDMS spectra. There is always residual water in these pressed sample pellets, even after baking, providing a ready source of $\mathrm{H}_{3} \mathrm{O}^{+}$. On the other hand, neutral $\mathrm{FeO}$ (IE 8.9 $\mathrm{eV}$ ) plays an important role in the formation of $\mathrm{FeO}^{+}$and $\mathrm{FeOH}^{+}$cluster ions. ${ }^{10}$ The following mechanisms would be expected to occur in the GD plasma:

$$
\begin{aligned}
& \mathrm{FeO}+\mathrm{Ar}^{*} \rightarrow \mathrm{FeO}^{+}+\mathrm{Ar}+\mathrm{e}^{-} \\
& \mathrm{FeO}+\mathrm{ArH}^{+} \rightarrow \mathrm{FeOH}^{+}+\mathrm{Ar}
\end{aligned}
$$

Neutral $\mathrm{FeO}$ is produced by direct sputtering, Fe and $\mathrm{O}$ recombination after sputtering, and $\mathrm{Fe}_{\mathrm{x}} \mathrm{O}_{\mathrm{y}}$ cluster fragmentation, but the ratio between these will vary with experimental conditions. However, oxygen abundance in iron oxide should 
relate directly to neutral $\mathrm{FeO}$ abundance and in turn relate to $\mathrm{FeO}^{+}$and $\mathrm{FeOH}^{+}$ intensity. Therefore, by comparing $\mathrm{FeO}$ related peak $\left(\mathrm{FeO}^{+}\right.$and $\left.\mathrm{FeOH}^{+}\right)$intensities with iron related peak $\left(\mathrm{Fe}^{+}\right.$and $\left.\mathrm{FeOH}_{3}{ }^{+}\right)$intensities, it is possible to discriminate the three iron oxides.

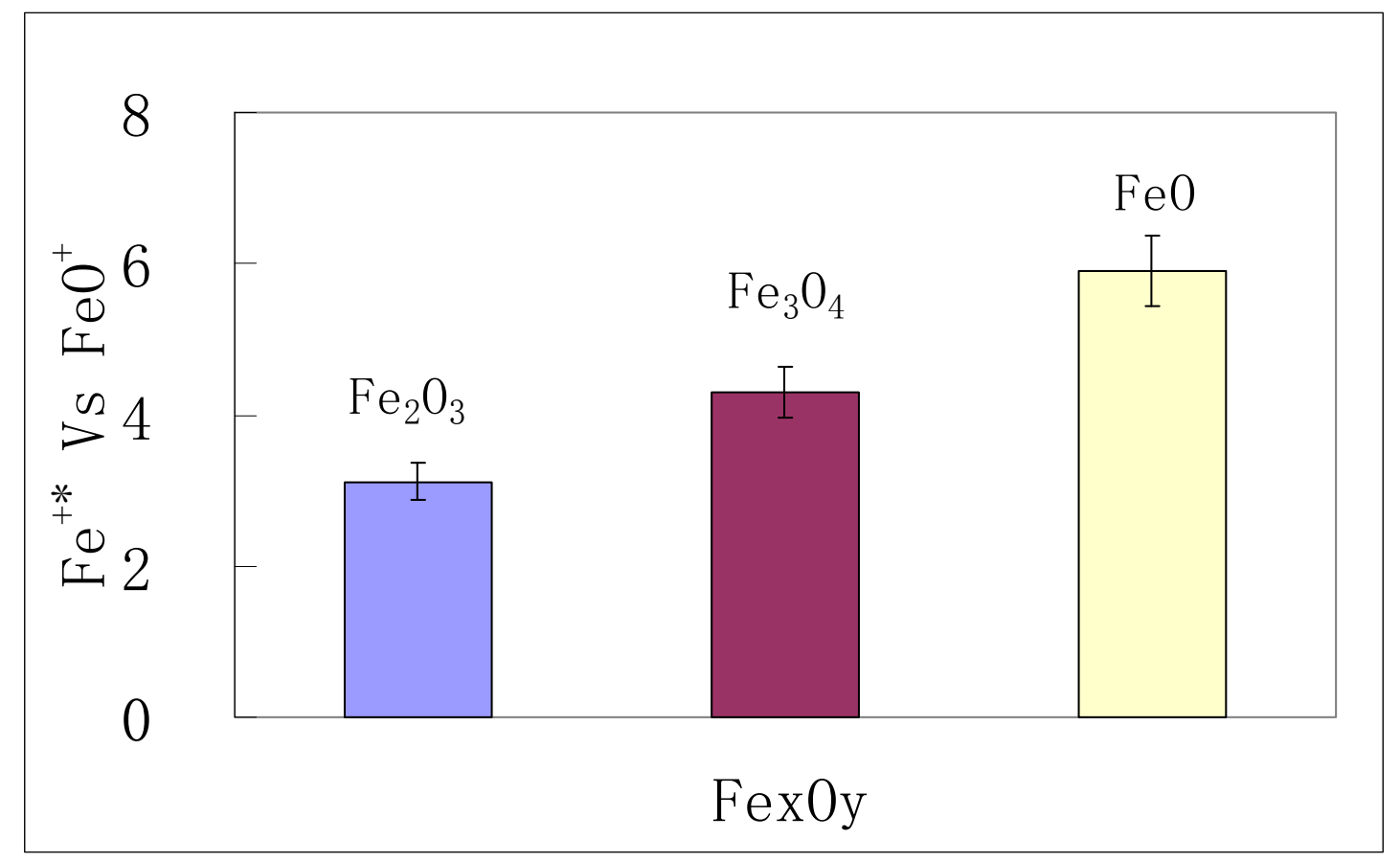

Figure 3.10 $\mathrm{Fe}$ and $\mathrm{FeO}$ related peak intensity ratios for three iron oxides. Glow discharge operating power $80 \mathrm{~W}$ with $2 \mathrm{~ms}$ pulse width and $10 \%$ duty cycle, gas pressure 0.3 torr, sampling distance $15 \mathrm{~mm}$. Each data point is the result of 6 measurements and error bars represent one standard deviation from the mean value.

* $\mathrm{Fe}^{+}$includes $\mathrm{Fe}^{+}$and $\mathrm{Fe}-\mathrm{OH}_{3}^{+}$.

Figure 3.10 shows the result of this ratio comparison and is consistent with 
the mechanisms identified above. The $\mathrm{Fe}_{2} \mathrm{O}_{3}$ sample, which has highest oxygen abundance, shows lowest value (3.115) among three; whereas the $\mathrm{FeO}$ sample, which has lowest oxygen abundance, has highest value (5.906) and the $\mathrm{Fe}_{3} \mathrm{O}_{4}$ sample is in the middle (4.293).

\subsection{Conclusion}

The pulse width does not have obvious effect on mass spectra, while duty cycle settings affects ion signal intensity. By carefully tuning these parameters, a specific desired plasma environment can be produced to generate ions used for speciation. Ion intensity ratios ultimately enabled discrimination between $\mathrm{FeO}$, $\mathrm{Fe}_{2} \mathrm{O}_{3}$, and $\mathrm{Fe}_{3} \mathrm{O}_{4}$ samples. Because oxygen is related to $\mathrm{FeOH}^{+}$generation, this ratio is found to decrease as oxygen abundance increases. 


\subsection{References}

1. Al-Harahsheh, M.; Kingman, S.; Somerfield, C.; Ababneh, F. Microwave-Assisted Total Digestion of Sulphide Ores for Multi-Element Analysis. Anal. Chim. Acta 2009, 638(1), 101-105.

2. Qi, L.; Zhou, M.-F.; Wang, C. Y. Determination of Low Concentrations of Platinum Group Elements in Geological Samples by ID-ICP-MS. J. Anal. At. Spectrom. 2004, 19(10), 1335-1339.

3. F. Aubriet, C. Poleunis and P. Bertrand, Capabilities of Static ToFSIMS in the Differentiation of First-Row Transition Metal Oxides, J. Mass Spectrom., 2001, 36, $641-651$.

4. Na Zhang and Fred L. King, Direct Manganese (Mn) Speciation in Solid State Materials by Pulsed Glow Discharge Time-of-Flight Mass Spectrometry, J. Anal. At. Spectrom. 2009, 24(11), 1489-1497.

5. Torres Deluigi, M.; Tirao, G.; Stutz, G.; Cusatis, C.; Riveros, J. A. Dependence with the Oxidation State of X-Ray Transition Energies, Intensities, and Natural Line Widths of CrK_ Spectra. Chem. Phys. 2006, 325(2/3), 477-484.

6. Bin Yan, Lingfeng Li, Quan Yu, Wei Hang, Jian He, and Benli Huang, High Irradiance Laser Ionization Mass Spectrometry for Direct Speciation of Iron Oxides, J Am Soc Mass Spectrom. 2010, 21, 1227-1234.

7. Aubriet, F.; Maunit, B.; Muller, J.-F, Speciation of Chromium Compounds by Laser ablation/Ionization Mass Spectrometry and a Study of Matrix Effects. Int. J. Mass Spectrom. 2001, 209(1), 5-21. 
8. Aubriet, F.; Poleunis, C.; Muller, J.-F.; Bertrand, P. Laser Ablation and Secondary Ion Mass Spectrometry of Inorganic Transition-Metal Compounds. Part I: Comparison Between Static TOF-SIMS and LA-FTICRMS. J. Mass Spectrom. 2006, 41(4), 527-542.

9. Hachimi, A.; Van Vaeck, L.; Poels, K.; Adams, F.; Muller, J. F. Speciation of Chromium, Lead, and Nickel Compounds by Laser Microprobe Mass Spectrometry and Application to Environmental and Biological Samples. Spectrochim. Acta B 1998, 53(2), 347-365.

10. Maunit, B.; Hachimi, A.; Manuelli, P.; Calba, P. J.; Muller, J. F. Formation of Iron Oxides Clusters Induced by Resonant Laser Ablation/Ionization. Int. J. Mass Spectrom. Ion Processes 1996, 156(3), 173-187.

11. W. Hang, C. Baker, B. W. Smith, J. D. Winefordner and W. W. Harrison, Microsecond-pulsed Glow Discharge Time-of-flight Mass Spectrometry: Analytical Advantages, J. Anal. At. Spectrom., 1997, 12, 143-149.

12. R. K. Marcus, T. R. Harville, Y. Mei and C. R. Shick, Jr, Rf-Powered glow discharges. Elemental analysis, Anal. Chem., 1994, 66, 902A-911A.

13. L. Li, C. M. Barshick, J. T. Millay, A. V. Welty and F. L. King, Determination of Bromine in Flame-Retardant Plastics Using Pulsed Glow Discharge Mass Spectrometry, Anal. Chem., 2003, 75, 3953-3961.

14. C. L. Lewis, M. A. Moser, D. E. Dale, W. Hang, C. Hassell, F. L. King and V. Majidi, Time-Gated Pulsed Glow Discharge: Realtime Chemical Speciation at the 
Elemental, Structural, and Molecular Level for Gas Chromatography Time-of-Flight Mass Spectrometry, Anal. Chem., 2003, 75, 1983-1996.

15. S. J. Ray, F. Andrade, G. Gamez, D. McClenathan, D. Rogers, G. Schilling, W. Wetzel and G. M. Hieftje, Plasma-Source Mass Spectrometry for Speciation Analysis: State-of-the-Art. J. Chromatogr., A, 2004, 1050, 3-34.

16. Jennifer N. Robertson-Honecker, Na Zhang, Alexandria Pavkovichab and Fred L. King, Direct Chromium Speciation in Solid State Materials - a GDMS approach, $J$. Anal. At. Spectrom., 2008, 23, 1508-1517.

17. Lei Li, John T. Millay, John P. Turner, and Fred L. King, Millisecond Pulsed Radio Frequency Glow Discharge Time of Flight Mass Spectrometry: Temporal and Spatial Variations in Molecular Energetics, J Am Soc Mass Spectrom 2004, 15, $87-102$.

18. K. S. Molek, C. Anfuso-Cleary and M. A. Duncan, Photodissociation of Iron Oxide Cluster cations, J. Phys. Chem. A 2008, 112, 9238-9247.

19. Glen P. Jackson, Cris L. Lewis, Stephen K. Doorn, Vahid majidi, Fred L. King, Spectral, Spatial and Temporal Characteristics of a Millisecond Pulsed Glow Discharge: Metastable Argon Atom Production, Spectrochimica Acta Part B $56(2001)$ 2449-2464.

20. Cris L. Lewis, Glen P. Jackson, Stephen K. Doorn, Vahid Majidi, Fred L. King, Spectral, Spatial and Temporal Characteristics of a Millisecond Pulsed Glow Discharge: Copper Analyte Emission and Ionization, Spectrochimica Acta Part B 56(2001) 487-501. 


\section{Chapter 4}

\section{Extending the Application of Pulsed Millisecond Radio Frequency Glow Discharge Time-of-Flight Mass Spectrometry to the Characterization of Other Transition Metals (Co, Nb, and $\mathrm{Cu}$ )}

\subsection{Introduction}

As noted in the previous chapter, transition metal oxidation state determination is important because the oxidation state is directly related to the toxicity, matabolism, and transportation of the metal in the environment. A good example is chromium, whose toxicity varies greatly between $\mathrm{Cr}(\mathrm{III})$ and $\mathrm{Cr}(\mathrm{VI})$. The former is an essential nutrient in trace quantity (50 to $200 \mu \mathrm{g})$, while the latter is toxic and carcenogenic in humans. ${ }^{1,2}$

Previous works in our lab demonstrated that pulsed glow discharge mass spectrometry (GDMS) can also be used in transion metal oxides specitaion including manganese (II, IV), chromium (III, VI), and iron (II, III) oxides. In some cases solid state samples are succssfully discriminated based on specific ions. Qualitative and quantitative analysis was also possible with this technique. ${ }^{8,9}$ Experiments were developed in an effort to extend this method to other transition metal oxides. Three groups of metal oxides including Cobalt oxides $(\mathrm{CoO}$ and 
$\left.\mathrm{Co}_{3} \mathrm{O}_{4}\right)$, niobium oxides $\left(\mathrm{NbO}, \mathrm{NbO}_{2}\right.$, and $\left.\mathrm{Nb}_{2} \mathrm{O}_{5}\right)$, and copper oxides $\left(\mathrm{Cu}_{2} \mathrm{O}\right.$, and $\mathrm{CuO}$ ) were examined in the experiment. Previous ToF SIMS investigations found that cobalt and copper oxides yield characteristic ions. ${ }^{4}$

\subsection{Experimental}

Radio frequency power to the system is supplied by a $13.56 \mathrm{MHz}$ radio frequency $(\mathrm{RF})$ generator equipped with an automatic matching network (RF Plasma Products Inc., Marlton, NJ). Applied power, pulse width, and duty cycle can be controlled by the on-board CPU of the generator. Argon (ultra pure, Airgas, Randor, PA) was introduced into the glow discharge chamber as the discharge support gas through a metering valve and the pressure was monitored via a thermocouple pressure gauge (Varian, Lexington, MA). Analytical reagent grades of cobalt (II) oxide (95\%), cobalt (II, III) oxide (99\%), niobium (II) oxide (purity data not given), niobium (IV) oxide $(99 \%+)$, niobium (V) oxide $(99.5 \%)$, copper (I) oxide $\left(\mathrm{Cu}_{2} \mathrm{O}\right)$, and copper (II) oxide $(\mathrm{CuO})$ were purchased from commercial suppliers (Alfa Aesar, Ward Hill, MA and Aldrich, St. Louis, MO). All metal oxides were mixed with spectroscopic grade silver powder (99.999\%, Alfa Aesar, Ward Hill, MA) at different concentrations by weighing. Niobium oxides were also mixed with analytical reagent grades copper powder (99.9\%, Alfa Aesar, Ward Hill, MA) at different concentration by weighting. Approximately $0.6 \mathrm{~g}$ of mixed powders were homogenized for 3 min using a laboratory mixer (Wig-L-Bug, SPEX, Edison, NJ). Each mixture was then compacted in a die assembly 
constructed in the department workshop under 4000 psi for 30 min to yield sample disks $5 \mathrm{~mm}$ in diameter and $\sim 6 \mathrm{~mm}$ in height. Both metal oxide powders and pressed pellets are baked overnight at $110^{\circ} \mathrm{C}$ in oven before use. Sample disks were introduced on the tip of the direct insertion probe (DIP) and served as the glow discharge cathode. The distance between the sample cathode and ion sampling orifice anode plate was adjusted by moving DIP axially. Cathode samples were shielded by a non-conductive MACOR $^{\circledR}$ shield (Accuratus, Washington, N.J.) to maintain cathode surface area and current density while restricting sputtering to sample top surface. The Time-of-Flight Mass Spectrometry operating settings are listed in Table 4.1. and further information can be found in previous papers regarding this system. ${ }^{8,9}$

Table 4.1 Pulsed RF GD TOF-MS Operating Parameters

\begin{tabular}{|lc|}
\hline Glow discharge & \\
Pressure & $0.3 \mathrm{Torr}$ \\
Operating power & $60-120 \mathrm{~W}$ \\
Pulse frequency & $50 \mathrm{~Hz}$ \\
Duty cycle & $10,25 \%$ \\
Sampling distance & $7-17 \mathrm{~mm}$ \\
Time-of-flight & \\
Flight path length & $1 \mathrm{~m}$ \\
Ion lenses potential & \\
Skimmer & $-350 \mathrm{~V}$ \\
Accelerator(A2) & $-1535 \mathrm{~V}$ \\
Deflector (X1) & $-1830 \mathrm{~V}$ \\
Deflector (Y1) & $-1535 \mathrm{~V}$ \\
Repeller(A1) & $200 \mathrm{~V}$ \\
Detector & $-1850 \mathrm{~V}$ \\
\hline
\end{tabular}




\subsection{Result and Discussion}

\subsubsection{Optimization of Temporal Regimes}

Glow discharge plasmas can work both in continuous and pulsed modes. The pulsed glow discharge has two advantages over the continuous glow discharge. First, a power-off period after power-on period will allow cathode to cool down and alleviate overheating. Therefore, a higher instantaneous power can be applied to enhance sputtering, excitation, and ionization processes in the glow discharge. Second, pulsed operation enables temporal discrimination of sample signals and background noise as a result of different ionization processes happening during different time regimes over a pulse cycle.

When a repeating square wave form RF power is supplied to glow discharge, three temporal regimes are observed: prepeak, plateau, and afterpeak. In the prepeak period, electron ionization is the only source of ionization and sputtering has not begun. Only those species present in the gas phase before power-on are ionized and detected, sample cathode species have not yet been introduced into the plasma. Spectra of prepeak yield no analytical information for this reason and will not be discussed.

During the plateau period, ions are formed by multiple processes, chiefly electron ionization, Penning ionization, and charge transfer ionization. Because both "hard" and "soft" ionization occur in plateau period, atomic ions, cluster ions, and 
molecular ions can be expected to be seen in the spectra. Figure 4.1 shows mass spectra from pure $\mathrm{Ag}, 25 \% \mathrm{CoO} / \mathrm{Ag}$, and $25 \% \mathrm{Co}_{3} \mathrm{O}_{4} / \mathrm{Ag}$ pellets acquired in the plateau period. In the pure silver sample spectrum, argon ions and silver isotope ions are the principal species observed. In spectra acquired for cobalt oxides mixed with silver pellets, the cobalt atomic ion $\left(\mathrm{Co}^{+}, \mathrm{m} / \mathrm{z} 59\right)$ is detected as well. Unfortunately, no ions characteristic for either cobalt oxide are observed. Similar observations were found for niobium oxides and copper oxides. It is clear from this that the plateau period will not provide information that enables the differentiation between the different forms of these metal oxides. This is consistent with previous observations for other metal oxides that this group has examined.

(a) Pure Ag

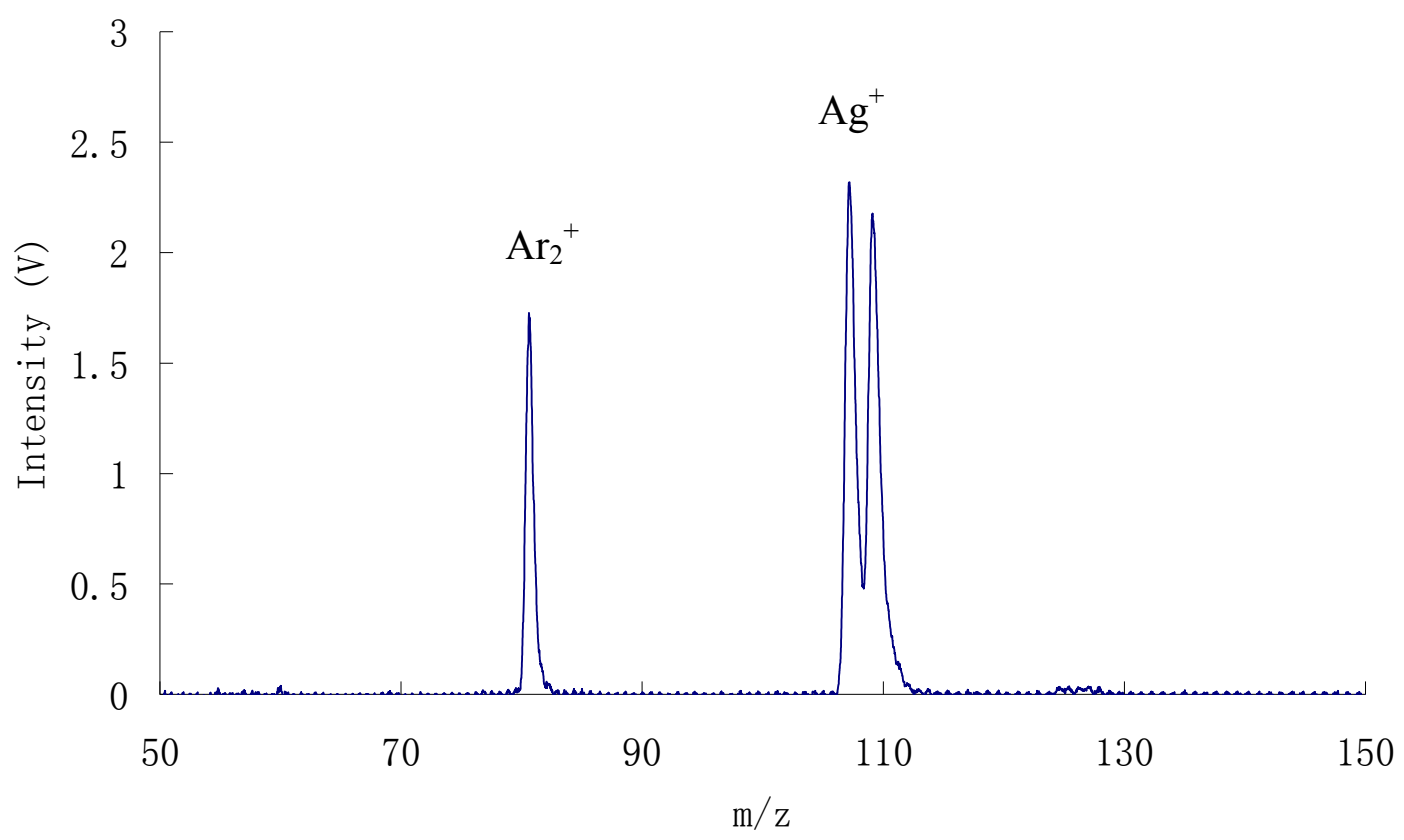


(b) $25 \% \mathrm{CoO} / \mathrm{Ag}$

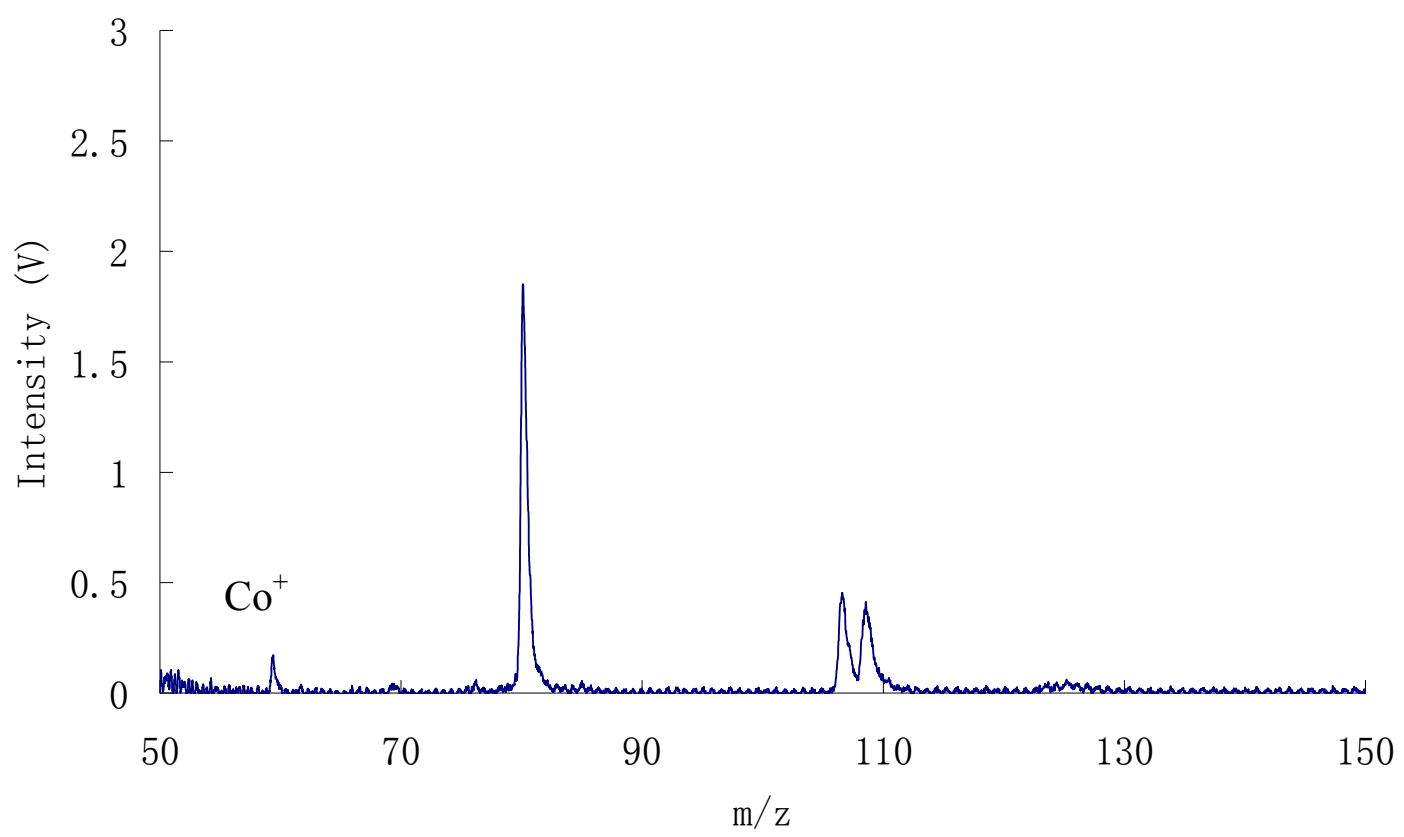

(c) $25 \% \mathrm{Co}_{3} \mathrm{O}_{4} / \mathrm{Ag}$

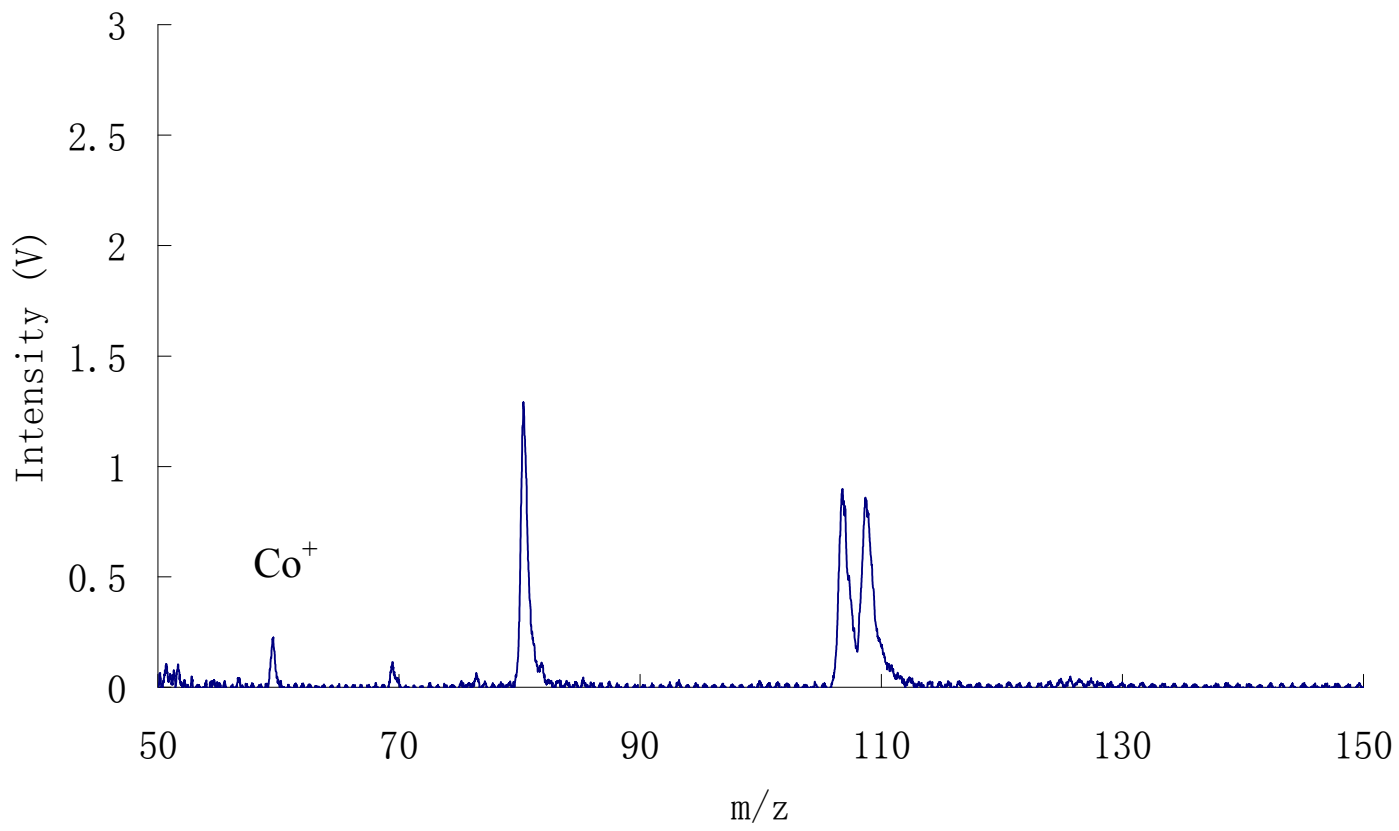

Figure 4.1 Time resolved mass spectra of (a) pure $\mathrm{Ag}$, (b) $25 \% \mathrm{CoO} / \mathrm{Ag}$, and (c) $25 \%$ $\mathrm{Co}_{3} \mathrm{O}_{4} / \mathrm{Ag}$ during the plateau (2.0 ms after power-on). Discharge pressure 0.3 Torr, peak power $80 \mathrm{~W}$, frequency $50 \mathrm{~Hz}$, duty cycle $10 \%$ duty cycle and sampling distance $10 \mathrm{~mm}$. 
The afterpeak period is dominated by the "soft" Penning ionization process. This arises because after the termination of applied power, fast electrons lose kinetic energy quickly through collision processes in the chamber. The resultant thermalized electrons can then recombine with argon ions and form energetic metastable argon atoms. The energy of metastable argon atoms are 11.55 and $11.62 \mathrm{eV}$. Energy transfers between metastable argon atoms and species in the plasma having lower ionization potentials will generate ions of those species. The selectivity of Penning ionization decreases background noise as contaminants in glow discharge plasmas have generally greater ionization potentials than the metastable argon atom energy. Because a large population of metastable argon atoms is generated in this period, more analyte ions are formed in afterpeak than in plateau.

In analytical pulsed glow discharge mass spectrometry, the afterpeak period supplies the most useful information for speciation purposes. Figure 4.2 shows mass spectra of pure $\mathrm{Ag}, 25 \% \mathrm{CoO} / \mathrm{Ag}$, and $25 \% \mathrm{Co}_{3} \mathrm{O}_{4} / \mathrm{Ag}$ pellets collected at $0.2 \mathrm{~ms}$ after power-off. In the spectrum from a pure silver sample, silver peak intensities are nearly twice as large as they were in the plateau (Figure 4.1). Silver water cluster ions $\left(\mathrm{Ag}-\mathrm{H}_{2} \mathrm{O}^{+}\right)$are also observed. In the spectra from cobalt oxides mixed with silver, the intensity for the atomic cobalt ion $\left(\mathrm{Co}^{+}, \mathrm{m} / \mathrm{z} 59\right)$ also increases significantly. Cobalt oxides cluster ions can also be found in the spectra, such as $\mathrm{CoO}^{+}(\mathrm{m} / \mathrm{z} 75)$ and $\mathrm{CoOH}^{+}\left(\mathrm{m} / \mathrm{z}\right.$ 76). A weak cobalt oxide water cluster ion $\left(\mathrm{CoO}-\mathrm{H}_{2} \mathrm{O}^{+}\right)$signal is also detected. 
Cluster ions have been demonstrated to be useful in both qualitative and quantitative analysis for manganese and chromium oxides. Robertson found $\mathrm{Cr}_{2} \mathrm{O}^{+}$ and $\mathrm{CrO}_{3}{ }^{+}$were specific for Chromium (III) oxide $\left(\mathrm{Cr}_{2} \mathrm{O}_{3}\right)$ and Chromium (VI) oxide $\left(\mathrm{CrO}_{3}\right)$ samples respectively. ${ }^{8}$ Zhang found that the $\mathrm{Mn}_{2} \mathrm{O}_{3}{ }^{+}$ion is unique for Manganese (IV) oxide $\left(\mathrm{MnO}_{2}\right) .{ }^{9}$ It was anticipated that cluster ions characteristic of the Co species might also be found to exist for optimized conditions. 
(a) Pure Ag

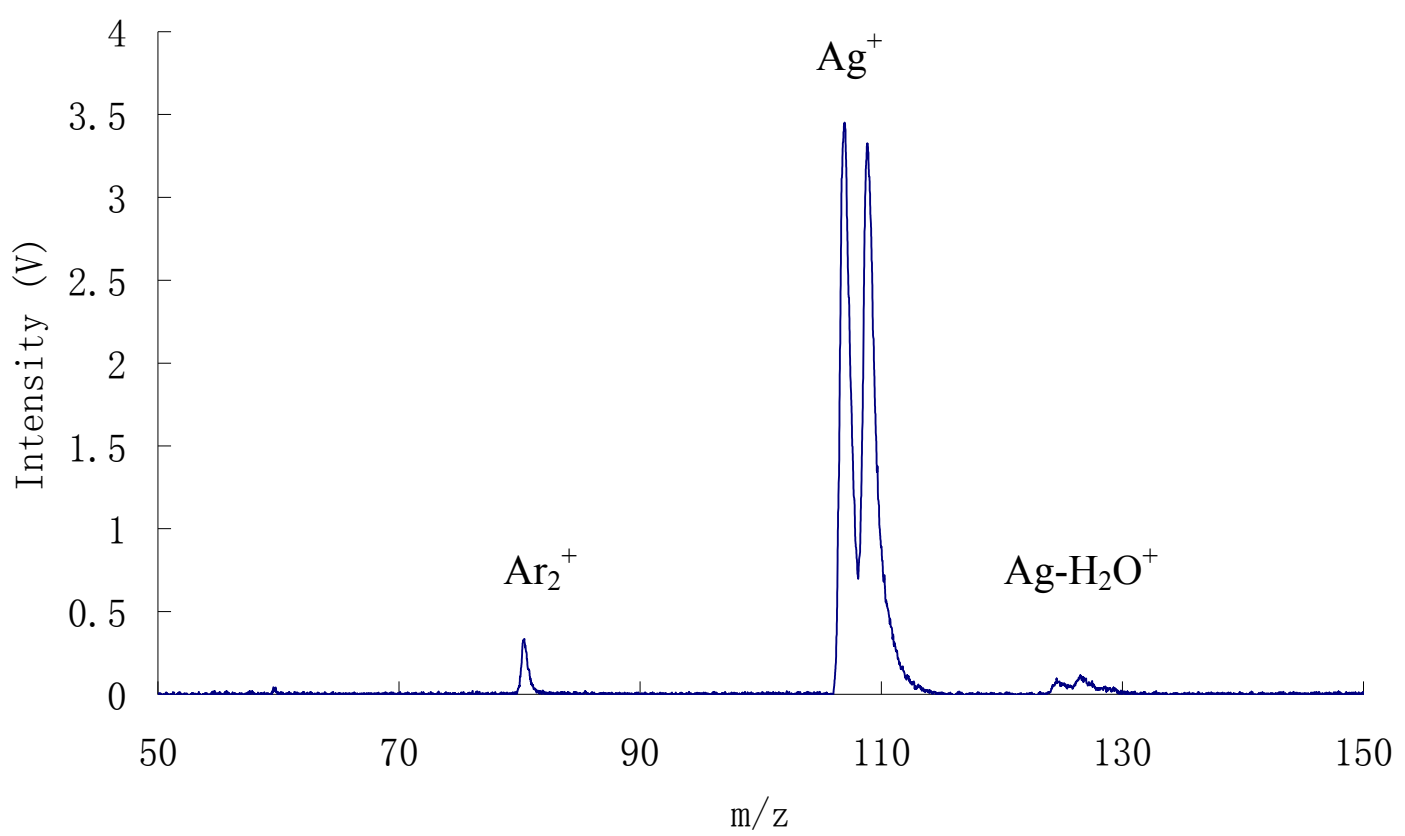

(b) $25 \% \mathrm{CoO} / \mathrm{Ag}$

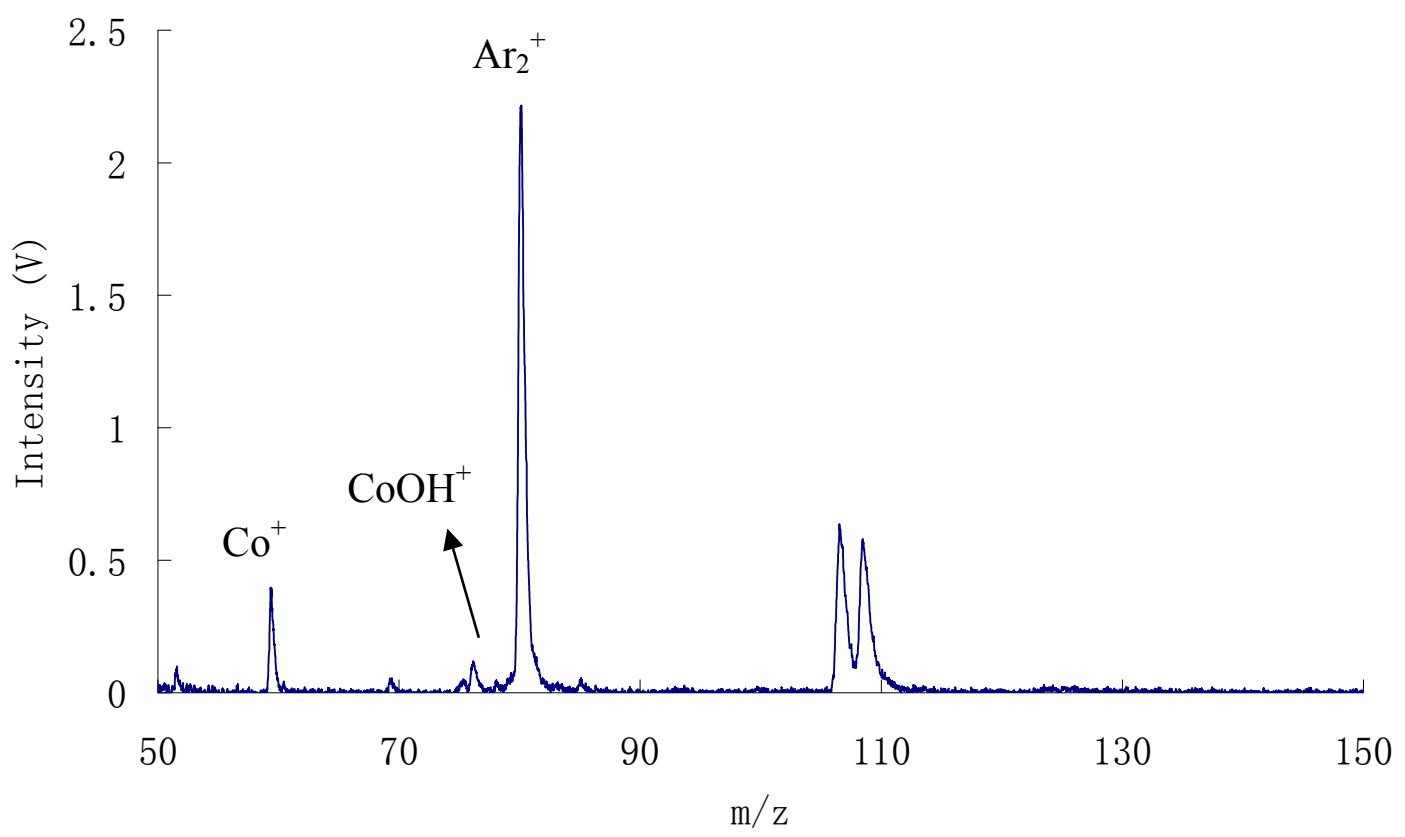


(c) $25 \% \mathrm{Co}_{3} \mathrm{O}_{4} / \mathrm{Ag}$

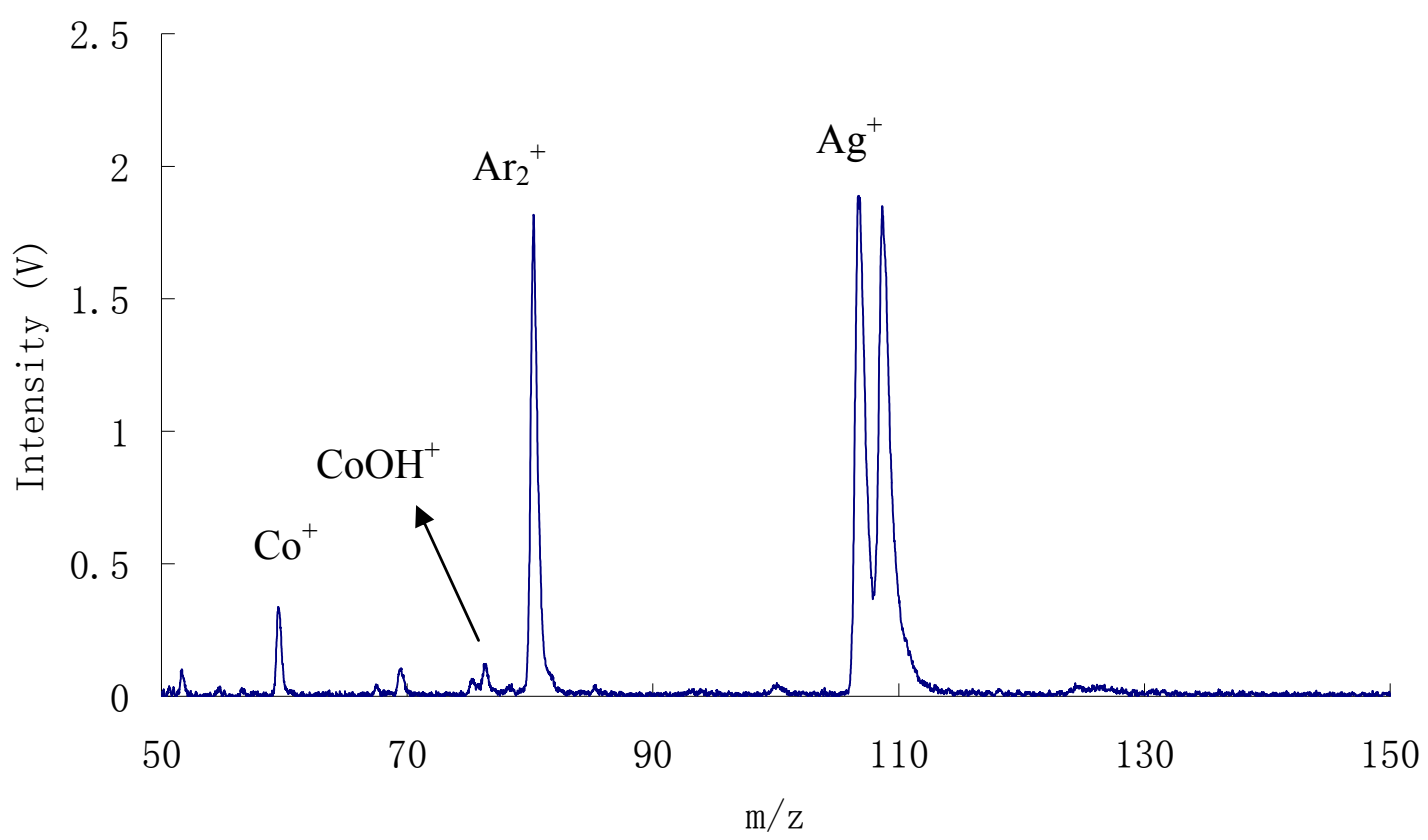

Figure 4.2 Time resolved mass spectra of (a) pure $\mathrm{Ag}$, (b) $25 \% \mathrm{CoO} / \mathrm{Ag}$, and (c) $25 \%$ $\mathrm{Co}_{3} \mathrm{O}_{4} / \mathrm{Ag}$ samples during the afterpeak (0.2 ms after power-off). Discharge pressure 0.3 Torr, peak power $80 \mathrm{~W}$, frequency $50 \mathrm{~Hz}, 10 \%$ duty cycle and sampling distance $10 \mathrm{~mm}$.

\subsubsection{Optimization of Sampling Distance}

Based on previous work done in our lab, different species will have different spatial distributions in the glow discharge. ${ }^{8-11}$ This is because ionization and excitation of analytes in these glow discharges is spatially dependent. Exploration of this spatial distribution should enable optimization of sampling distance to enhance analytical signals.

Figure 4.3 shows the influence of sampling distance on ion signals from a $25 \%$ 
$\mathrm{CoO} / \mathrm{Ag}$ pellet. Mass spectra are collected in the afterpeak period at $0.2 \mathrm{~ms}$ after power-off. The discharge gas (argon) pressure is 0.3 Torr; operating power is $80 \mathrm{~W}$; frequency is $50 \mathrm{~Hz}$; duty cycle is $10 \%$. The sampling distance is measured between the cathode surface and sampling orifice. By moving the probe position, sampling distance is changed from $7 \mathrm{~mm}$ to $17 \mathrm{~mm}$. The figure shows that $\mathrm{Co}^{+}(\mathrm{m} / \mathrm{z} 59)$ and $\mathrm{Ag}^{+}(\mathrm{m} / \mathrm{z} 107$ and 109) intensities keep on growing from $7 \mathrm{~mm}$ to $11 \mathrm{~mm}$ and then begin to drop. In the dropping process, $\mathrm{Ag}^{+}$intensity drops faster than $\mathrm{Co}^{+} \cdot \mathrm{CoOH}^{+}$ $(\mathrm{m} / \mathrm{z} 76)$ intensity reaches maximum earlier than the atomic ions at $9 \mathrm{~mm}$, then decreases slowly as distance increases. To get satisfactory signals for both atomic and cluster ions, $10 \mathrm{~mm}$ is selected as the optimum sampling distance.

\subsubsection{Optimization of Operating Power}

As discussed in the first chapter, there are three parameters needed to describe a pulsed glow discharge's power: peak power, frequency, and duty cycle. For optimization, peak powers ranging from $60 \mathrm{~W}$ to $120 \mathrm{~W}$ were investigated. It was found that peak powers lower than $60 \mathrm{~W}$, failed to generate ion signals of sufficient intensity for analytical use, whereas peak powers greater than $120 \mathrm{~W}$ led to plasma instability. The sample used in this study is a $25 \% \mathrm{Co}_{3} \mathrm{O}_{4} / \mathrm{Ag}$ pellet. Mass spectra are collected in the afterpeak period at $0.2 \mathrm{~ms}$ after power-off. The discharge gas pressure is 0.3 Torr Argon, the sampling distance is $10 \mathrm{~mm}$, and pulse frequency is $50 \mathrm{~Hz}$ with a $10 \%$ duty cycle. 


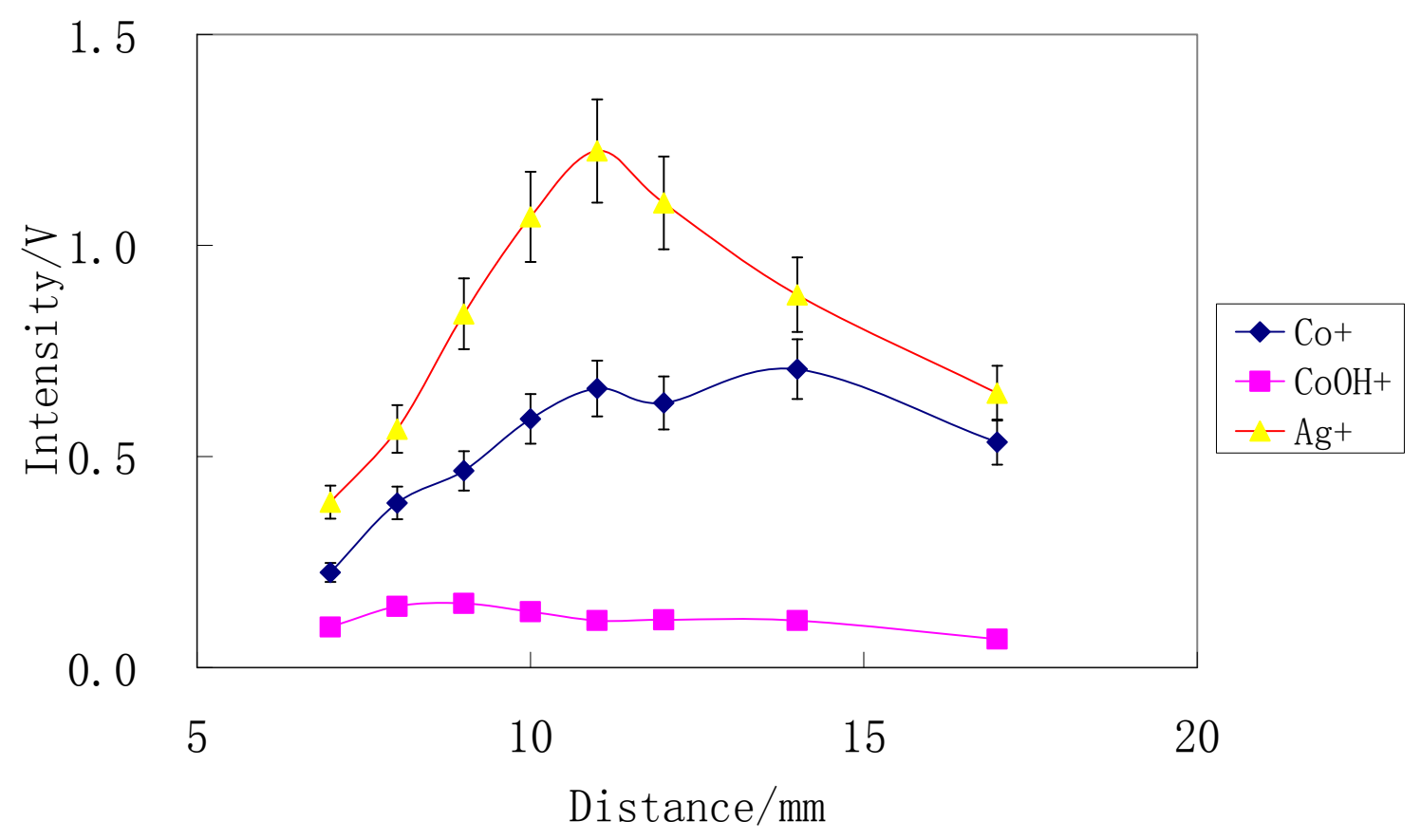

Figure 4.3 Spatial effect on ion signals for $25 \% \mathrm{CoO} / \mathrm{Ag}$ pellet. Data is colleted in afterpeak period at $0.2 \mathrm{~ms}$ after power-off. The discharge gas pressure is 0.3 Torr; peak power is $80 \mathrm{~W}$ with $50 \mathrm{~Hz}$ frequency and $10 \%$ duty cycle. Each data point is the result of 3 measurements and error bars represent one standard deviation from the mean value. 


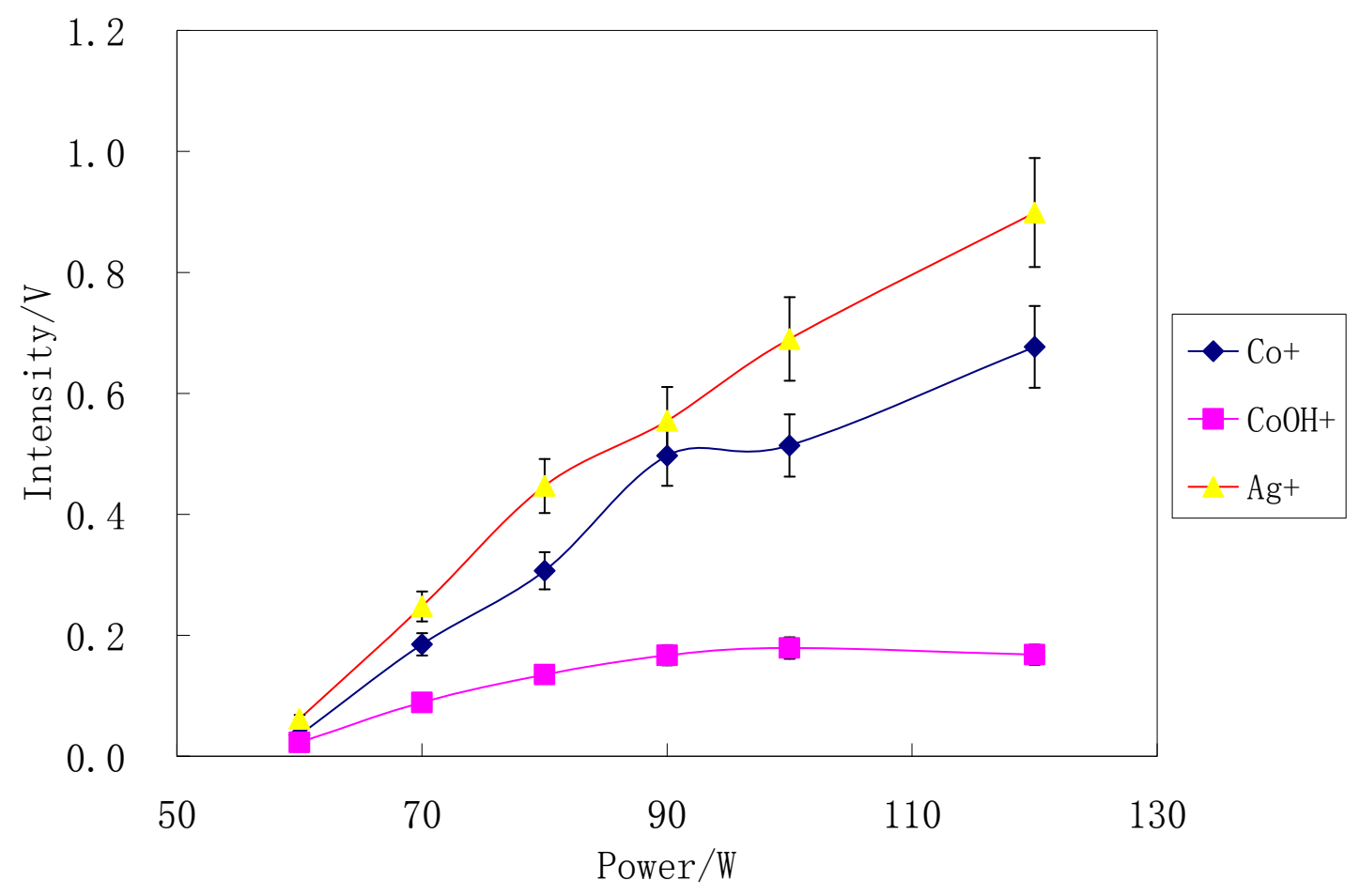

Figure 4.4 Power effect on ion signals for $25 \% \mathrm{Co}_{3} \mathrm{O}_{4} / \mathrm{Ag}$ sample pellet. Data are colleted in afterpeak period at $0.2 \mathrm{~ms}$ after power-off. The discharge gas pressure is 0.3 Torr; the sampling distance is $10 \mathrm{~mm}$; pulse frequency is $50 \mathrm{~Hz}$ with $10 \%$ duty cycle. Each data point is the result of 3 measurements and error bars represent one standard deviation from the mean value.

Figure 4.4 shows that atomic ions $\left(\mathrm{Co}^{+}\right.$and $\left.\mathrm{Ag}^{+}\right)$exhibit similar trends with increasing power. When peak power increases, their ion intensities increase dramatically. This can be explained by higher cathode sputter yield and better ionization efficiency that accompanies power increases. On the other hand, cluster ion $\left(\mathrm{CoOH}^{+}\right)$intensity peaks at $90-100 \mathrm{~W}$ and then levels off. To get stable signal for both atomic and cluster ions as well as to compare with previous work on this instrument, $80 \mathrm{~W}$ is selected as the peak power for the rest of the experiments. 


\subsubsection{Transition Metal Oxides Analysis}

\subsubsection{Cobalt Oxides Analysis}

In Aubreit's experiment, $\mathrm{CoO}$ has two specific cluster ions $\mathrm{Co}_{2} \mathrm{O}_{2}{ }^{-}$and $\mathrm{Co}_{7} \mathrm{O}_{7}{ }^{-}$; $\mathrm{Co}_{3} \mathrm{O}_{4}$ generates one specific cation $\mathrm{Co}_{5} \mathrm{O}_{5}{ }^{+}$and one specific anion $\mathrm{Co}_{7} \mathrm{O}_{9}{ }^{-4}$. As mentioned before, anions are not observable in our glow discharge mass spectrometer, so this work focuses on cluster cations.

Figure 4.2 shows mass spectra of $25 \% \mathrm{CoO} / \mathrm{Ag}$ and $25 \% \mathrm{Co}_{3} \mathrm{O}_{4} / \mathrm{Ag}$ in the afterpeak period. Several cobalt related ions, such as $\mathrm{Co}^{+}(\mathrm{m} / \mathrm{z} 59), \mathrm{CoO}^{+}(\mathrm{m} / \mathrm{z} 75)$, $\mathrm{CoOH}^{+}\left(\mathrm{m} / \mathrm{z}\right.$ 76), and weak $\mathrm{CoOH}-\mathrm{H}_{2} \mathrm{O}^{+}(\mathrm{m} / \mathrm{z}$ 94), are detected. Compared with Aubreit's spectra, clusters generated in the glow discharge for cobalt oxides are much smaller. One reason for this is that the environment is more energetic even in the glow discharge afterpeak than in SIMS. Large clusters formed under this condition will break apart to small cluster or atoms as a result of excess internal energy or collision induced dissociation (CID). Because no specific cluster ions are detected, two cobalt oxides cannot be told apart based on glow discharge mass spectra.

\subsubsection{Niobium Oxides Analysis}

Three niobium oxides, Niobium (II) Oxide (NbO), Niobium (IV) Oxide $\left(\mathrm{NbO}_{2}\right)$, and Niobium (V) Oxide $\left(\mathrm{Nb}_{2} \mathrm{O}_{5}\right)$, are examined in this experiment. To avoid possible interference of $\mathrm{Ag}^{+}\left(\mathrm{m} / \mathrm{z} 107\right.$ and 109) with $\mathrm{NbO}^{+}$(m/z 109), niobium oxides are mixed with copper powder instead of silver powder. Figure 4.5 shows afterpeak mass 
spectra of $25 \%$ niobium oxides/copper pellets collected in the afterpeak period at 5.2 ms. Unlike the cobalt oxides, which generated small clusters only, larger cluster ions such as $\mathrm{NbO}_{3}{ }^{+}$are detected. Atomic ions and smaller cluster ions are also detected including $\mathrm{Nb}^{+}, \mathrm{NbO}^{+}$, and $\mathrm{NbO}_{2}^{+}$. Although the $\mathrm{NbO}_{2}$ pellet yields much higher ion intensities compared with $\mathrm{NbO}$ and $\mathrm{Nb}_{2} \mathrm{O}_{5}$, no cluster ions characteristic of each niobium oxide are detected.

\subsubsection{Copper Oxides Analysis}

There are two kinds of copper oxides tested in this experiment: $\mathrm{Cu}$ (II) oxide (cupric oxide, $\mathrm{CuO}$ ) and $\mathrm{Cu}(\mathrm{I})$ oxide (cuprous oxide, $\mathrm{Cu}_{2} \mathrm{O}$ ). Mass spectra of these two copper oxides in the afterpeak period are shown in Figure 4.6. Both pellets yield $\mathrm{Cu}^{+}\left(\mathrm{m} / \mathrm{z} 63\right.$ and 65) and weak $\mathrm{Cu}-\mathrm{H}_{2} \mathrm{O}^{+}\left(\mathrm{m} / \mathrm{z} 81\right.$ and 83) ions. One of the $\mathrm{Cu}-\mathrm{H}_{2} \mathrm{O}^{+}$ peaks $(\mathrm{m} / \mathrm{z} 81)$ is interfered by relative strong $\operatorname{Ar}_{2}^{+}(\mathrm{m} / \mathrm{z} 80)$ signal, and mass resolution is not sufficient to tell them apart.

Different from Aubriet and co-workers' observation with large copper-oxygen cluster ions, $\mathrm{Cu}_{4} \mathrm{O}_{2}{ }^{+}, \mathrm{Cu}_{5} \mathrm{O}_{2}{ }^{+}, \mathrm{Cu}_{4} \mathrm{O}_{3,4^{-}}$, and $\mathrm{Cu}_{5} \mathrm{O}_{3,4}$, in $\mathrm{CuO}$ sample, ${ }^{4}$ no specific copper oxide cluster ions are detected in either $\mathrm{Cu}_{2} \mathrm{O}$ or $\mathrm{CuO}$. Therefore, two copper oxides cannot be discriminated based on glow discharge mass spectra. 
(a) $25 \% \mathrm{NbO} / \mathrm{Cu}$

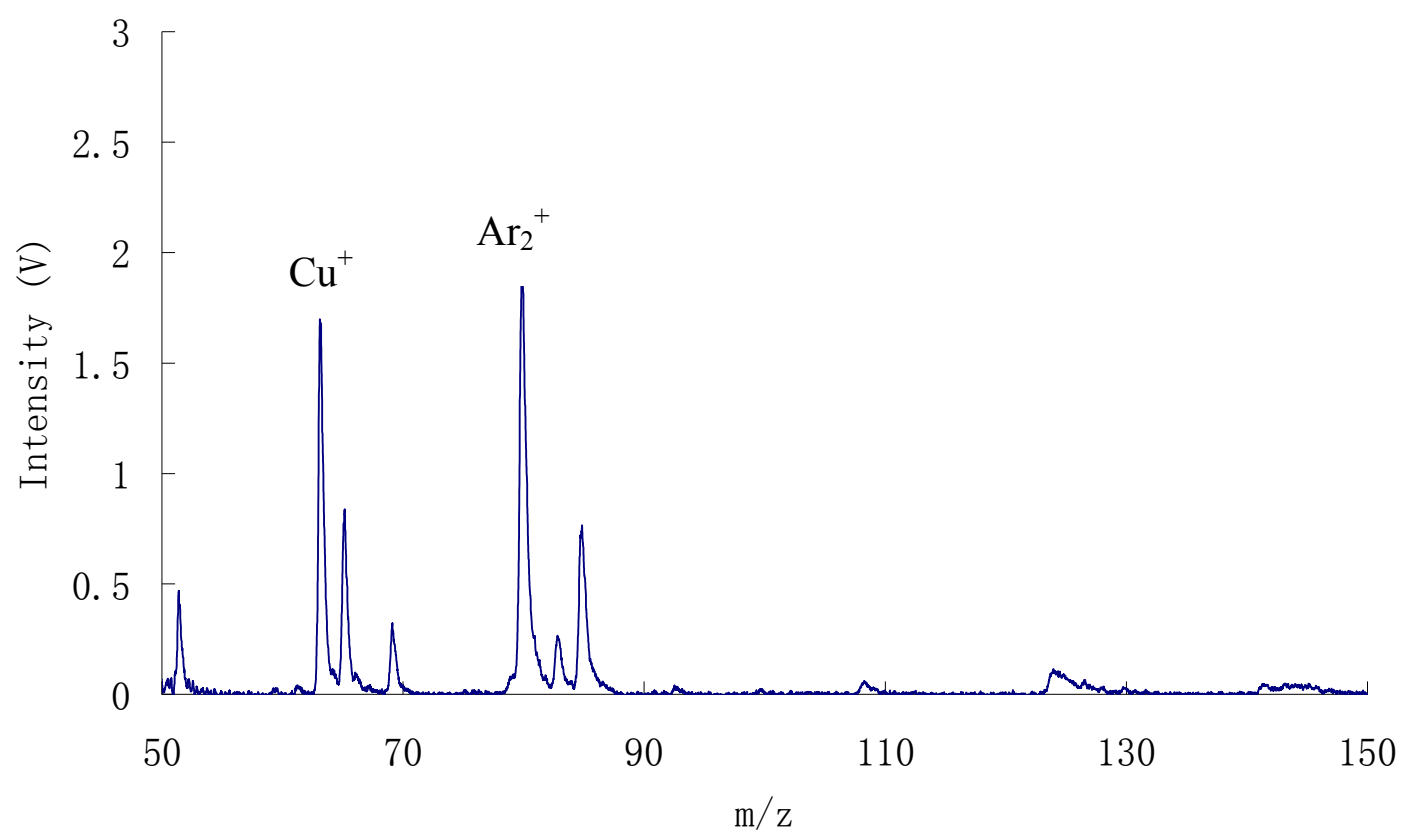

(b) $25 \% \mathrm{NbO}_{2} / \mathrm{Cu}$

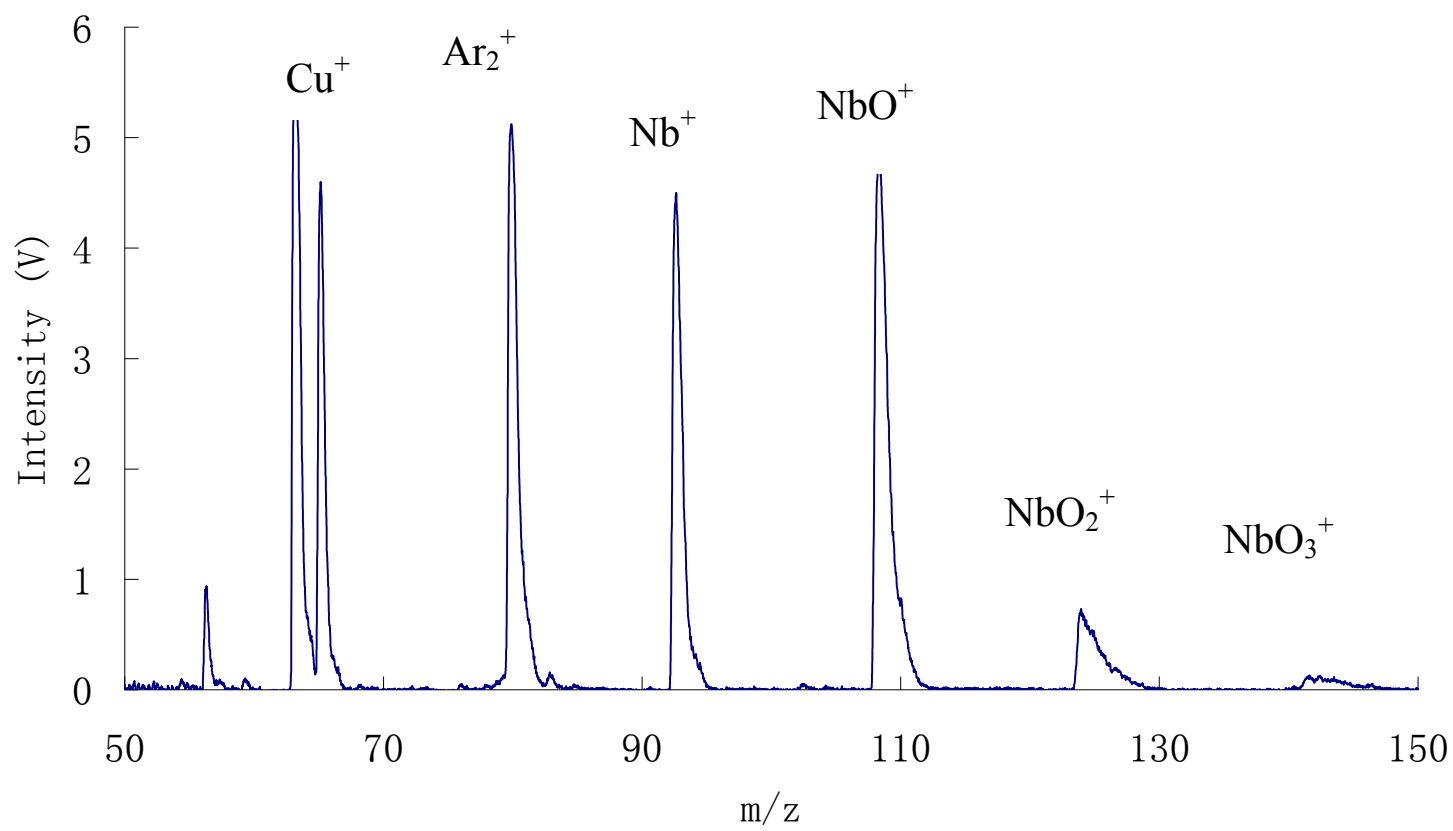


(c) $25 \% \mathrm{Nb}_{2} \mathrm{O}_{5} / \mathrm{Cu}$

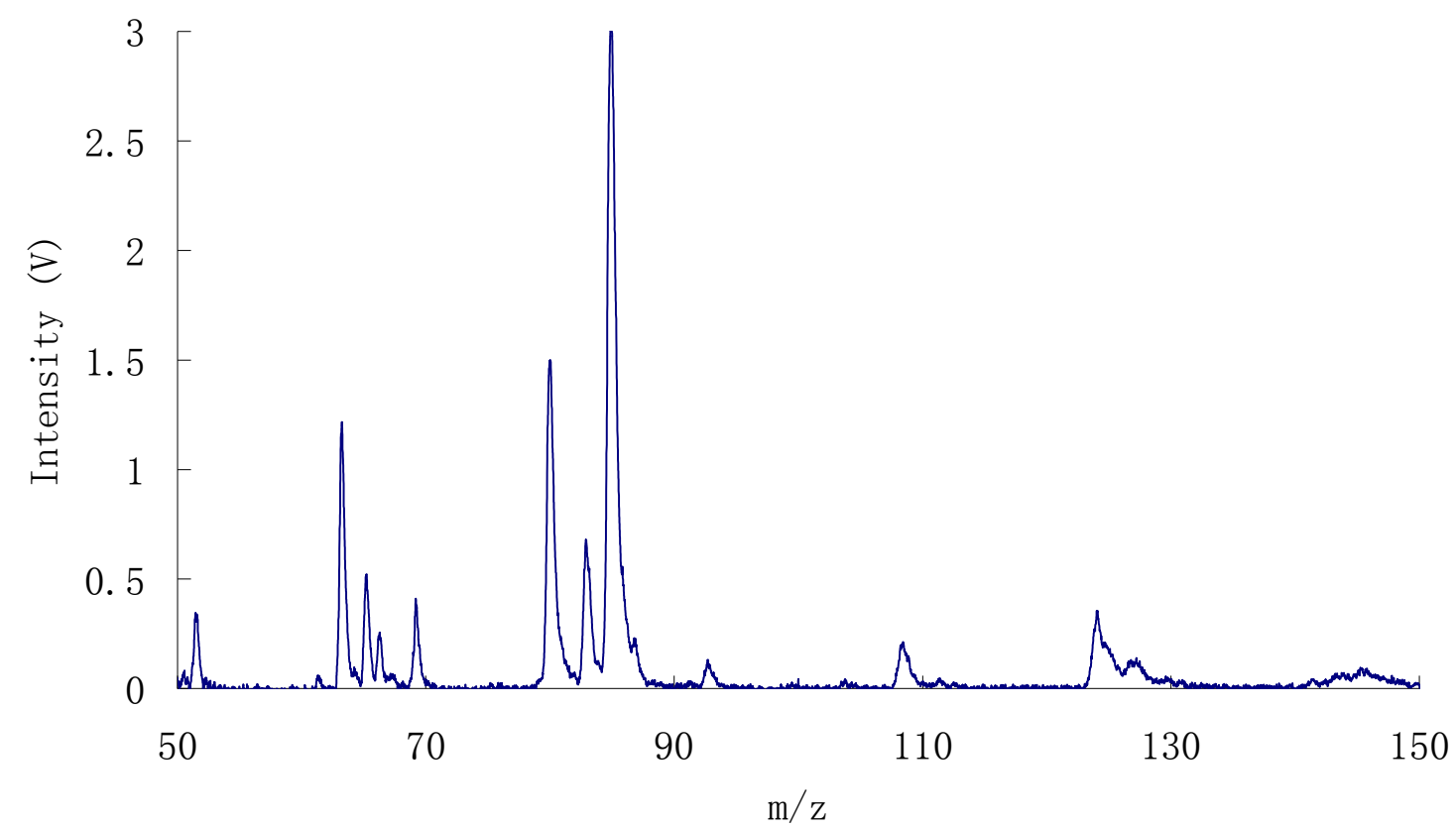

Figure 4.5 Mass spectra of (a) $25 \% \mathrm{NbO} / \mathrm{Cu}$ (b) $25 \% \mathrm{NbO}_{2} / \mathrm{Cu}$, and $25 \% \mathrm{Nb}_{2} \mathrm{O}_{5} / \mathrm{Cu}$ pellets during the afterpeak $(5.2 \mathrm{~ms})$. The discharge gas pressure is $0.3 \mathrm{Torr}$; the sampling distance is $10 \mathrm{~mm}$; pulse frequency is $50 \mathrm{~Hz}$ with $10 \%$ duty cycle. Each data point is the result of 3 measurements and error bars represent one standard deviation from the mean value. 
(a) $25 \% \mathrm{Cu}_{2} \mathrm{O}$ and $\mathrm{Ag}$

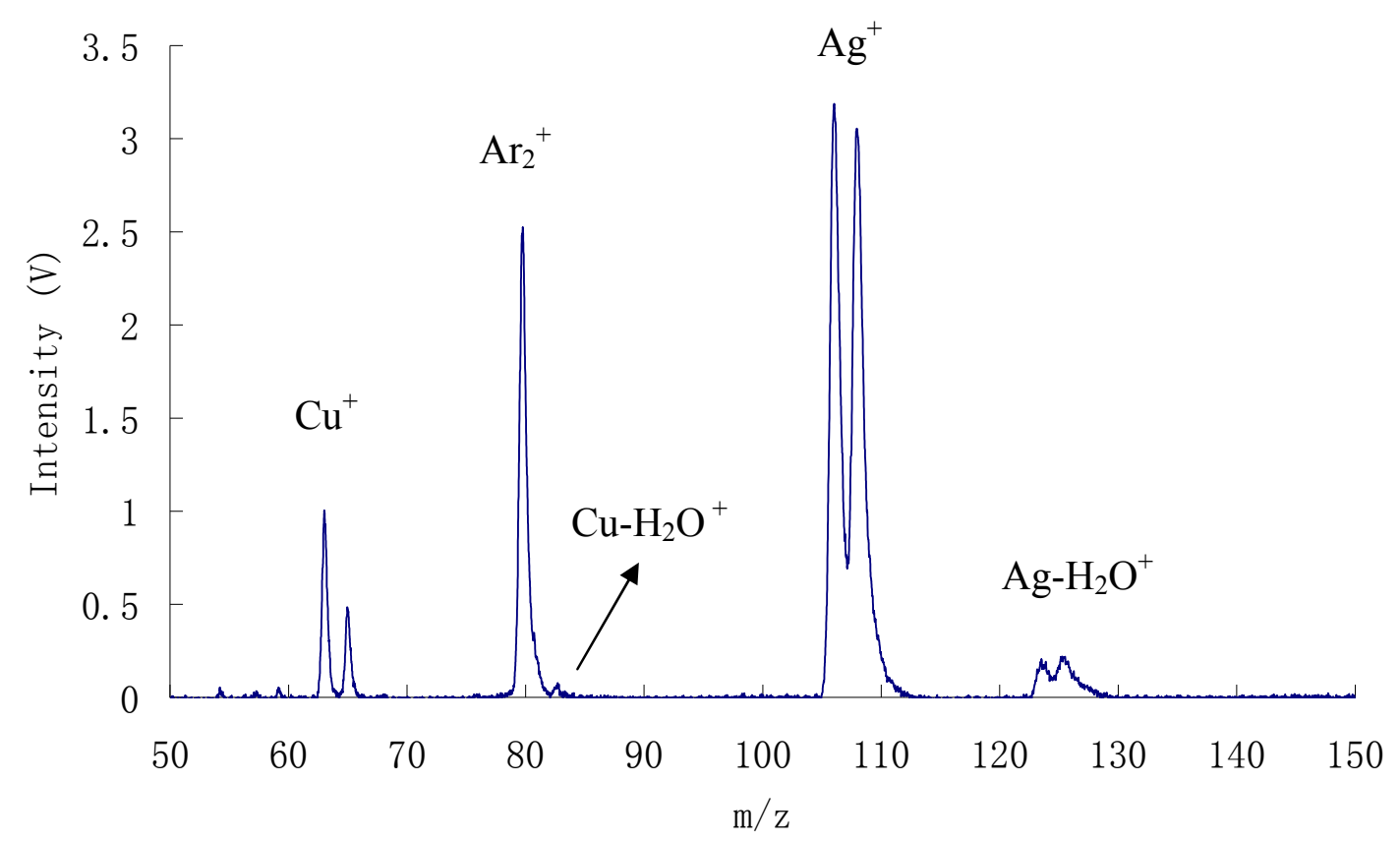

(b) $25 \% \mathrm{CuO}$ and $\mathrm{Ag}$

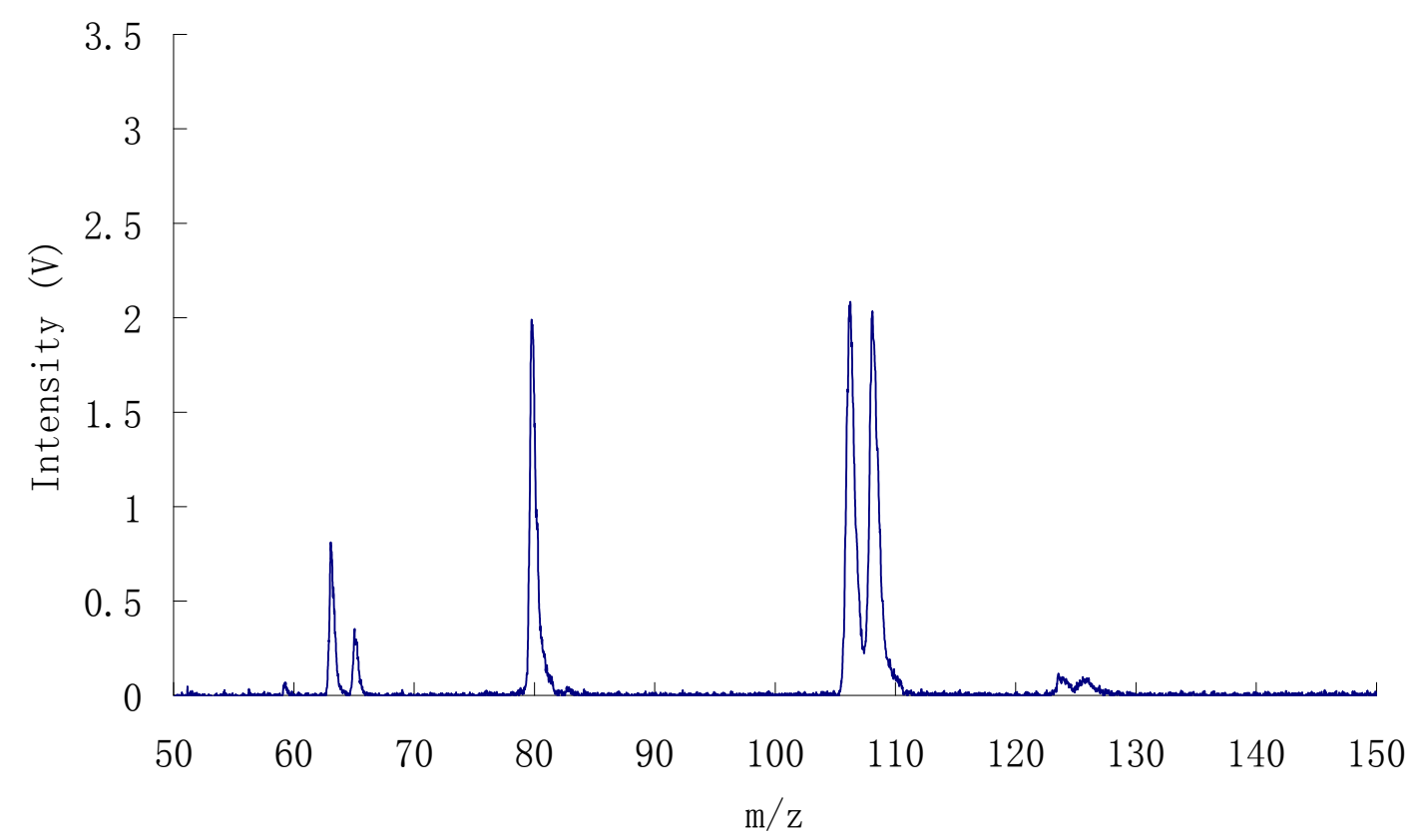

Figure 4.6 Mass spectra of (a) $25 \% \mathrm{Cu}_{2} \mathrm{O}$ and $\mathrm{Ag}$ (b) $25 \% \mathrm{CuO}$ and $\mathrm{Ag}$ pellets during the afterpeak $(5.1 \mathrm{~ms})$. Discharge pressure 0.3 Torr, peak power $80 \mathrm{~W}$, frequency 50 $\mathrm{Hz}$, duty cycle $25 \%$ duty cycle and sampling distance $10 \mathrm{~mm}$. 


\subsection{Conclusion}

This research was intended to extend our speciation technique to other transition metal oxides of $\mathrm{Co}, \mathrm{Nb}$, and $\mathrm{Cu}$. Three groups of metal oxides including Cobalt oxides $\left(\mathrm{CoO}\right.$ and $\left.\mathrm{Co}_{3} \mathrm{O}_{4}\right)$, niobium oxides $\left(\mathrm{NbO}, \mathrm{NbO}_{2}\right.$, and $\left.\mathrm{Nb}_{2} \mathrm{O}_{5}\right)$, and copper oxides $\left(\mathrm{Cu}_{2} \mathrm{O}\right.$, and $\left.\mathrm{CuO}\right)$ were tested after optimization of experimental conditions to determine if characteristic clusters could be found. Although each group has different kinds of cluster ions formed, no specific cluster ion is found within the group to enable speciation. Additional approaches need to be developed for the speciation of these transition metal oxides. 


\subsection{References}

1. T. O. Berner, M. M. Murphy and R. Slesinski, Determining the Safety of Chromium Tripicolinate for Addition to Foods as a Nutrient Supplement, Food

Chem. Toxicol., 2004, 42, 1029-1042.

2. M. Valko, H. Morris and M. T. D. Cronin, Metals, Toxicity, and O xidative Stress, Curr. Med. Chem., 2005, 12, 1161-1208.

3. A. Hachimi, E. Poitevin, G. Krier, J. F. Muller and F. Ruiz-Lopez, Study of the Mechanism of Chromium Cluster Formation by Laser Microprobe Mass Spectrometry. Correlation with Theoretical Computations. Int. J. Mass Spectrom.and Ion Processes, 1995, 144, 23-45.

4. F. Aubriet, C. Poleunis and P. Bertrand, Capabilities of Static ToFSIMS in the Differentiation of First-Row Transition Metal Oxides, J. Mass Spectrom., 2001, 36, $641-651$.

5. Aubriet, F.; Maunit, B.; Muller, J.-F, Speciation of Chromium Compounds by Laser ablation/Ionization Mass Spectrometry and a Study of Matrix Effects. Int. J. Mass Spectrom. 2001, 209(1), 5-21.

6. Aubriet, F.; Poleunis, C.; Muller, J.-F.; Bertrand, P. Laser Ablation and Secondary Ion Mass Spectrometry of Inorganic Transition-Metal Compounds. Part I: Comparison Between Static TOF-SIMS and LA-FTICRMS. J. Mass Spectrom. 2006, 41(4), 527-542.

7. Hachimi, A.; Van Vaeck, L.; Poels, K.; Adams, F.; Muller, J. F. Speciation of Chromium, Lead, and Nickel Compounds by Laser Microprobe Mass 
Spectrometry and Application to Environmental and Biological Samples. Spectrochim. Acta B 1998, 53(2), 347-365.

8. Jennifer N. Robertson-Honecker, Na Zhang, Alexandria Pavkovichab and Fred L. King, Direct Chromium Speciation in Solid State Materials - a GDMS Approach, J. Anal. At. Spectrom., 2008, 23, 1508-1517.

9. Na Zhang and Fred L. King, Direct Manganese (Mn) Speciation in Solid State Materials by Pulsed Glow Discharge Time-of-Flight Mass Spectrometry, J. Anal. At. Spectrom. 2009, 24(11), 1489-1497.

10. Glen P. Jackson, Cris L. Lewis, Stephen K. Doorn, Vahid majidi, Fred L. King, Spectral, Spatial and Temporal Characteristics of a Millisecond Pulsed Glow Discharge: Metastable Argon Atom Production, Spectrochimica Acta Part B $56(2001) 2449-2464$

11. Cris L. Lewis, Glen P. Jackson, Stephen K. Doorn, Vahid Majidi, Fred L. King, Spectral, Spatial and Temporal Characteristics of a Millisecond Pulsed Glow Discharge: Copper Analyte Emission and Ionization, Spectrochimica Acta Part B 56(2001) 487-501.

12. Lei Li, John T. Millay, John P. Turner, and Fred L. King, Millisecond Pulsed Radio Frequency Glow Discharge Time of Flight Mass Spectrometry: Temporal and Spatial Variations in Molecular Energetics, J Am Soc Mass Spectrom 2004, 15, $87-102$. 


\section{Chapter 5}

\section{A Preliminary Exploration of Peptide and Amino Acid Identification by Pulsed Glow Discharge Mass Spectrometry}

\subsection{Introduction}

Direct analysis in solid sample without dissolution is the primary advantage of glow discharge mass spectrometry (GDMS). Bulk metals and semiconductors are common samples examined in those experiments. Both qualitative and quantitative analysis are achieved and excellent detection limits as low as ppb level was reported. ${ }^{1-3}$ Beyond this, GDMS has been shown to provide information for molecular species. McLuckey et al. used an air sampling (air also served as the discharge support gas) glow discharge ion source to determine trace impurities in atmospheric samples. ${ }^{4}$ Detection of explosives at the ppb level was reported. Lewis and co-workers coupled a pulsed GDMS with gas chromatography and performed analysis of several aromatic and halogenated hydrocarbons. ${ }^{5}$ This had an advantage over McLuckey's experiment in that mass spectra collected at different time regimes of the pulse supplied elemental, structural, and molecular information.

Solution analysis by GDMS was first reported by Harrison's group by depositing dropwise solution on cathode disk surface in $1976 .{ }^{6}$ Later on, 
Jakubowski and co-workers reported a sub-picogram level detection for platimum(Pt) and iridium(Ir) in solution with same sampling method. ${ }^{7}$ Marcus and co-workers did a lot work on coupling liquid chromatography with particle beam glow discharge mass spectrometry (LC-PB/GDMS). ${ }^{8-13}$ In their experiments, samples including organic, inorganic, and botanical extracts were nebulized by thermoconcentric nebulizer after liquid chromatographic separation. Most solvent particles were removed by a momentum separater and sample particles were introduced into plasma to be ionized. The acquired mass spectra were EI-like in appearance with both molecular and fragment ions.

Sputter vaporization arising from ion bombardment of the cathode surface in glow discharges is similar to that in secondary ion mass spectrometry (SIMS). The key difference between these two techniques is that SIMS utilizes ions directly sputtered from the analyte, whereas, glow discharge mass spectrometry utilizes ions formed in the plasma from neutrals sputtered from the analyte. Experiments performed on SIMS can provide starting points for new applications of GDMS. Benninghoven et al. reported the identification of a number of amino acids from argon ion SIMS. ${ }^{14}$ Both molecular and specific fragment ions were detected in those experiments which were the genesis for today's booming field of biological MS. Aberth and co-workers also reported successful analysis of peptide and lipid samples by SIMS and fast atom bombardment (FAB). ${ }^{15}$ By extension, it should be possible to apply GDMS to bio-molecule analysis. 
To investigate the feasibility of a GDMS approach in bio-molecules analysis, a pulsed radio frequency glow discharge was applied to cathode surface, on which dropwise bio-molecules solution was deposited. Several amino acids were chosen based on side chain functional groups (Figure 5.1). Molecular and specific ions, which were seen in previous SIMS and FAB experiment, ${ }^{14,15}$ were expected to form and survive in the glow discharge plasma. A tripeptide was also tested. The results of this feasibility study that follow show some promise but also indicate that GDMS may be too energetic to allow this information to be obtained reliably.

\subsection{Experimental}

Radio frequency power to the system is supplied by a $13.56 \mathrm{MHz}$ radio frequency $(\mathrm{RF})$ generator equipped with an automatic matching network (RF Plasma Products Inc., Marlton, NJ). Peak power, pulse width, and duty cycle are controlled by the on-board CPU of the generator. Argon (ultra pure, Airgas, Randor, PA) was introduced into the glow discharge chamber as the discharge support gas through a metering valve and the pressure was monitored via a thermocouple pressure gauge (Varian, Lexington, MA).

Spectroscopic grade silver powder (99.999\%, Alfa Aesar, Ward Hill, MA) is baked overnight at $110{ }^{\circ} \mathrm{C}$ in oven before use. About $0.6 \mathrm{~g}$ of silver powder is compacted in a die assembly constructed in the department workshop under 4000 psi for 30 min to yield sample disks $5 \mathrm{~mm}$ in diameter and $\sim 6 \mathrm{~mm}$ in height. Pressed pellets are baked overnight at $110^{\circ} \mathrm{C}$ in oven before use. Glysine, leucine, 
serine, cysteine, tyrosine, histidine, and GlyGlyHis (99\%, Sigma Aldrich, St. Louis, MO) were prepared on the surface of sample disk in three different methods. In one method, each bio-molecule was mixed with glycerol (99\%, Fisher Scientific, Fairlawn, NJ) to form a $10 \mathrm{ppk}$ liquid mixture. $10 \mu \mathrm{l}$ of the liquid was then deposited on the surface of the disk before analysis. A second approach dissolve bio-molecules in deionized water to form saturated or 10 ppk solution at room temperature, whichever reaches first. The third method dissolves amino acids and GlyGlyHis in $10 \mathrm{mM} \mathrm{CsCl}$ (99.9\%, Sigma Aldrich, St. Louis, MO) solution to form saturated or $10 \mathrm{ppk}$ solution at room temperature, whichever reaches first. In the last two methods, $10 \mu \mathrm{l}$ of prepared solution was deposited to the disk surface and the disk was air-dried for 30 minutes before analysis .

Sample disks were introduced into the vacuum chamber on the tip of the direct insertion probe (DIP) and served as the glow discharge cathode. The distance between the sample cathode and ion sampling orifice anode plate was adjusted by moving the DIP axially. Cathode samples were shielded by a non-conductive $\mathrm{MACOR}^{\circledR}$ shield (Accuratus, Washington, N.J.) to maintain cathode surface area and current density while restricting sputtering to the top surface of the disk. The Time-of-Flight Mass Spectrometry operating settings are listed in Table 5.1 and further information can be found in previous papers regarding this system. ${ }^{17-19}$ 


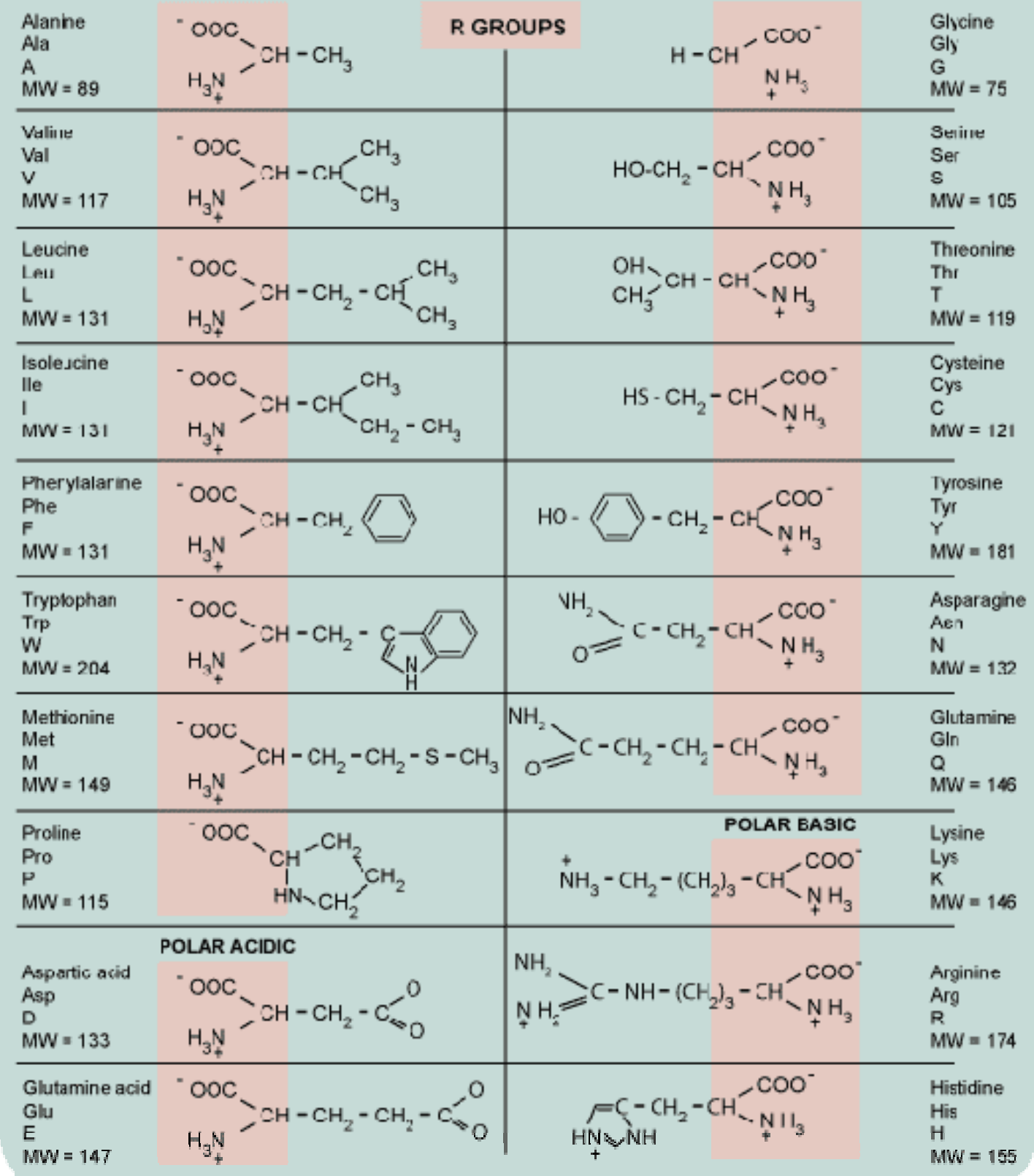

Figure 5.1 Structure of twenty amino acids. ${ }^{16}$ 
Table 5.1 Pulsed RF GD TOF-MS Operating Parameters

\begin{tabular}{|lc|}
\hline Glow discharge & \\
Pressure & $0.3 \mathrm{Torr}$ \\
Operating power & $80 \mathrm{~W}$ \\
Pulse frequency & $100 \mathrm{~Hz}$ \\
Duty cycle & $10 \%$ \\
Sampling distance & $15 \mathrm{~mm}$ \\
Time-of-flight & \\
Flight path length & $1 \mathrm{~m}$ \\
Ion lenses potential & \\
Skimmer & $-350 \mathrm{~V}$ \\
Accelerator(A2) & $-1535 \mathrm{~V}$ \\
Deflector (X1) & $-1830 \mathrm{~V}$ \\
Deflector (Y1) & $-1535 \mathrm{~V}$ \\
Repeller(A1) & $200 \mathrm{~V}$ \\
Detector & $-1850 \mathrm{~V}$ \\
\hline
\end{tabular}

\subsection{Result and Discussion}

As discussed in previous chapters, pulsed glow discharge has three time regimes within each pulse. Prepeak and plateau regimes did not provide useful inofrmation in these experiments, so discussion is limited to spectra collected in afterpeak regime.

\subsubsection{Sample and Glycerol Mixture Analysis}

Fast Atom Bombardment (FAB) and Liquid Secondary Ion Mass Spectrometry (LSIMS) can analyze liquid sample by focusing a high energy $(2-8 \mathrm{keV})$ primary beam of neutral atoms/molecules or ions on the sample and matrix mixtures. ${ }^{15,20}$ When these energetic primary particles impact the sample surface, the transfer of kinetic energy into matrix will eject ions and neutral particles from the solution. A 
similar sputtering process is expected to happen when matrix and samples are placed on the cathode surface in a glow discharge system. The key difference is that any cations are redeposited on the cathode surface by the strong electric field near the cathode. Sputtered neutrals are available for ionization in the plasma.

Figure 5.2 shows mass spectra of glycerol and glycerol-Gly mixture samples acquired during the afterpeak regime. In the glycerol spectrum, the protonated molecular ion $\left(\mathrm{MH}^{+}, \mathrm{m} / \mathrm{z} 93\right)$ is detected, but unlike the case for SIMS or $\mathrm{FAB}^{15}$, no larger glycerol cluster ions are observed. Some fragment ions are found between $\mathrm{m} / \mathrm{z}$ 50 and 150. The spectrum of the glycerol-Gly mixture is very similar to that of glycerol. Although the amino acid molecular ion was detected in SIMS experiment, neither molecular ion nor specific fragment ions are detected in the GDMS spectrum. Experiments with the other amino acids yielded similar results. 
(a) Glycerol

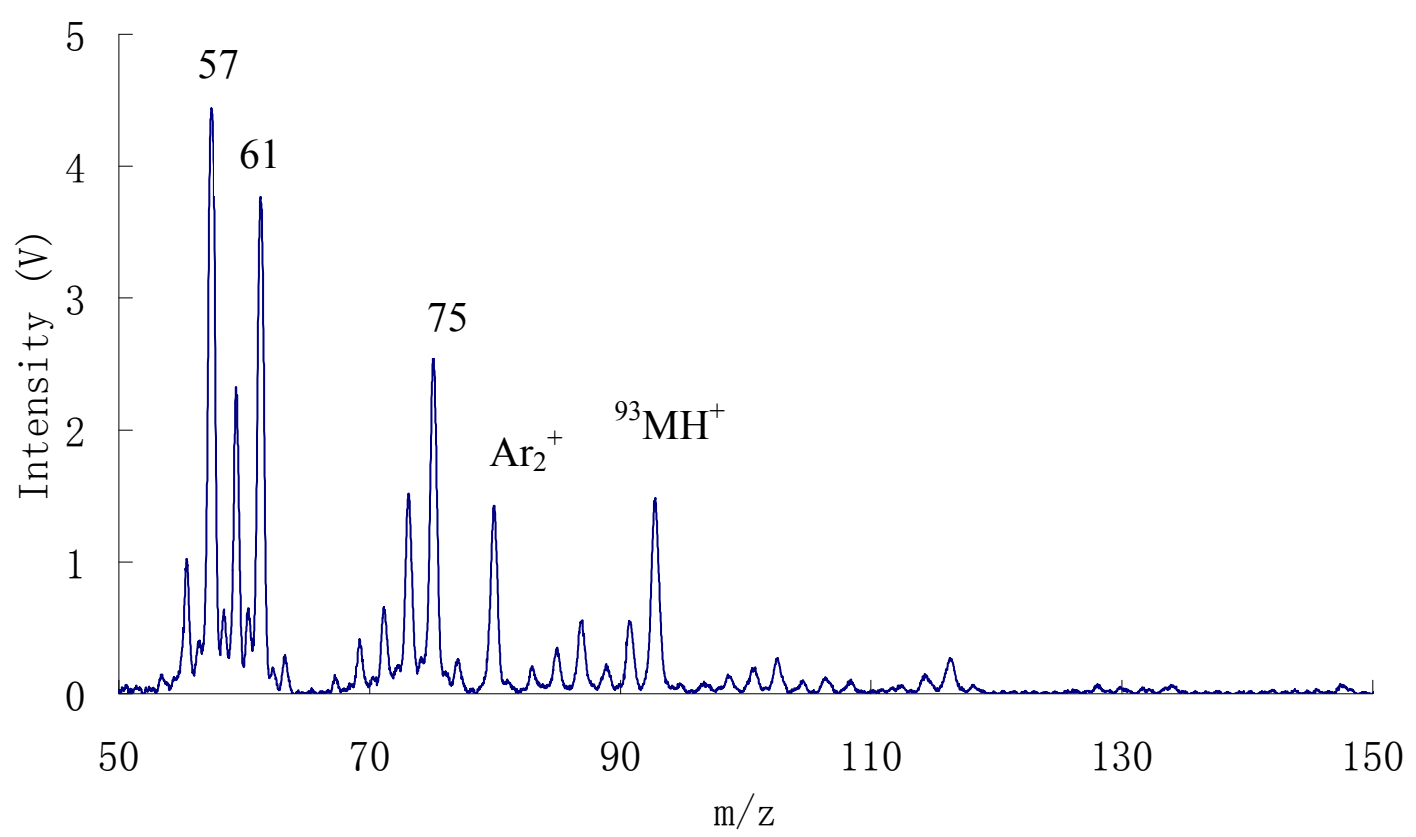

(b) Glycerol + Gly

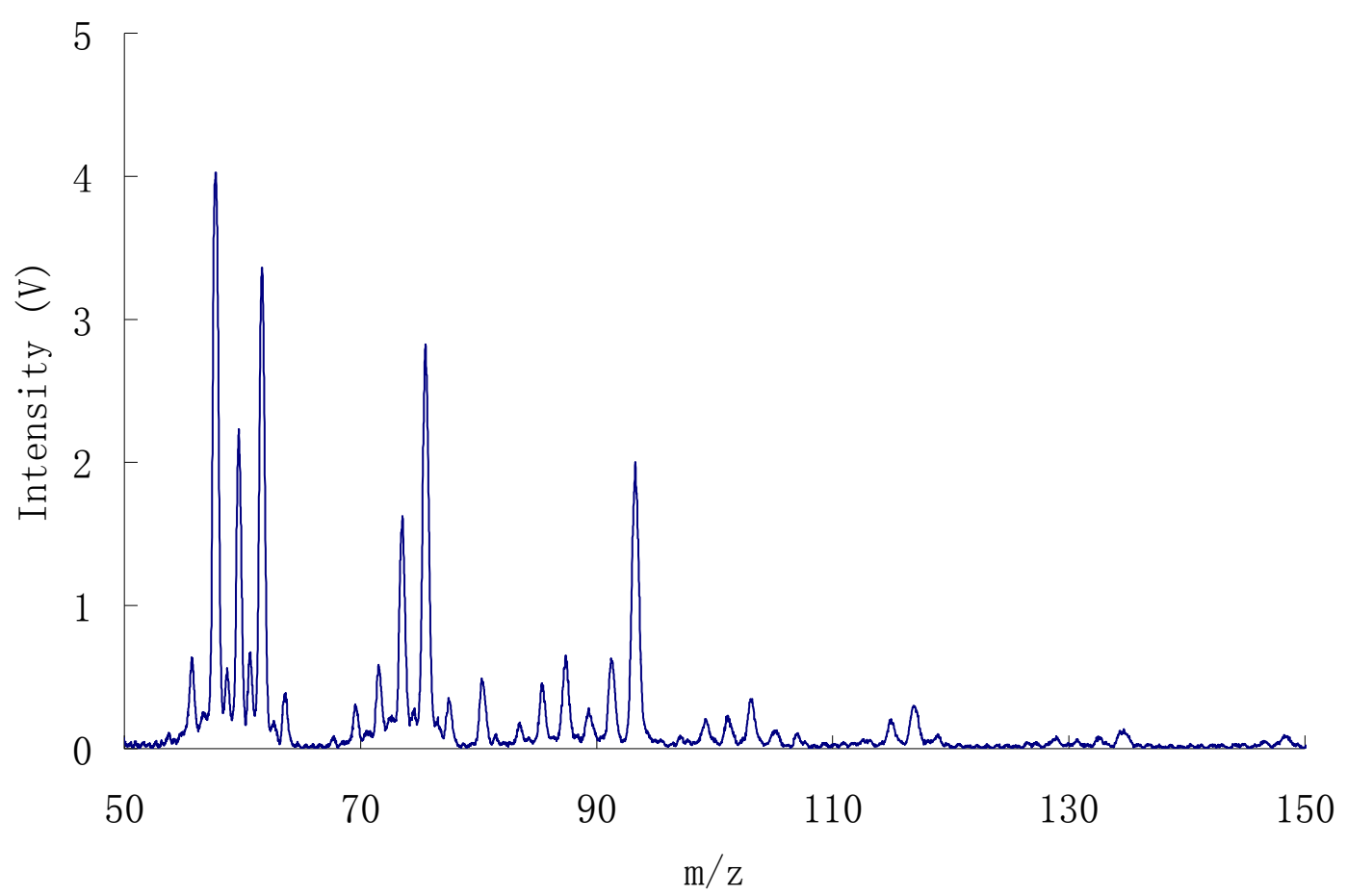

Figure 5.2 Mass spectra of (a) Glycerol and (b) Glycerol + Gly samples during the afterpeak (0.2 ms after power-off). Discharge pressure 0.3 Torr, sampling distance 15 mm, peak power $80 \mathrm{~W}$ with $100 \mathrm{~Hz}$ frequency and $10 \%$ duty cycle. 


\subsubsection{Sample Solution Analysis}

Direct solution analysis is not easy to achieve in GDMS, because nebulization of solvent molecules will quench the plasma. In other words, all or most of solvent has to be kept away from plasma. Among reported approaches for solution analysis by GDMS, the most used, and most convenient, method is to directly evaporate solution on the surface of the cathode. A thin layer of sample will coat on the surface after evaporation. Because of the small surface area of sample disk, only a limited amount of sample is tested in each run. Therefore, sample signal will gradually fade as the sputtering process goes on. In this experiment, all mass spectra were collected between 1 min and 3 min after glow discharge initiation - the initiation of sputtering.

The solution of the amino acids in deionized water was applied to the silver cathode surface, dried, and subjected to GDMS interrogation. The resultant mass spectra are presented in Figure 5.3. Deionized water is tested as the blank sample. It shows a similar mass spectrum to that from pure silver sample except for stronger silver water cluster ion signals, $\mathrm{Ag}-\mathrm{H}_{2} \mathrm{O}^{+}$at m/z 125 and 127 as well as some small cluster ion signals at $\mathrm{m} / \mathrm{z} 57,59$, and 61 . The mass spectrum of leucine is presented as a typical amino acid spectrum in this experiment. Several peaks appear between $\mathrm{m} / \mathrm{z}$ 50 and 60. Comparison with the blank sample indicates that some of these peaks arise the from amino acid backbone because they appear in spectra for every amino acid tested in the experiment. Generally, no molecular ion or characteristic fragment ions are observed for these amino acids. An exception is cysteine, which consistently 
provided a characteristic ion signal at $\mathrm{m} / \mathrm{z} 76$. Examination of the cysteine structure shown in Figure 5.1 indicates that the likely fragment ion arises from the loss of carboxylic acid. The strong peak intensity of this ion is also observed in the standard EI mass spectrum for cysteine (Figure 5.4) and in the SIMS experimet. ${ }^{14}$

One thought was that the energetic glow discharge leads to significant dissociation of these smaller molecules. To test this, a tripeptide, GlyGlyHis, was explored, in hopes that by having more available degrees of freedom for the energy to distribute over, fragmentation would be reduced. Unfortunately the resulting mass spectra did not exhibit any molecular ion or characteristic ions. 
(a) Silver

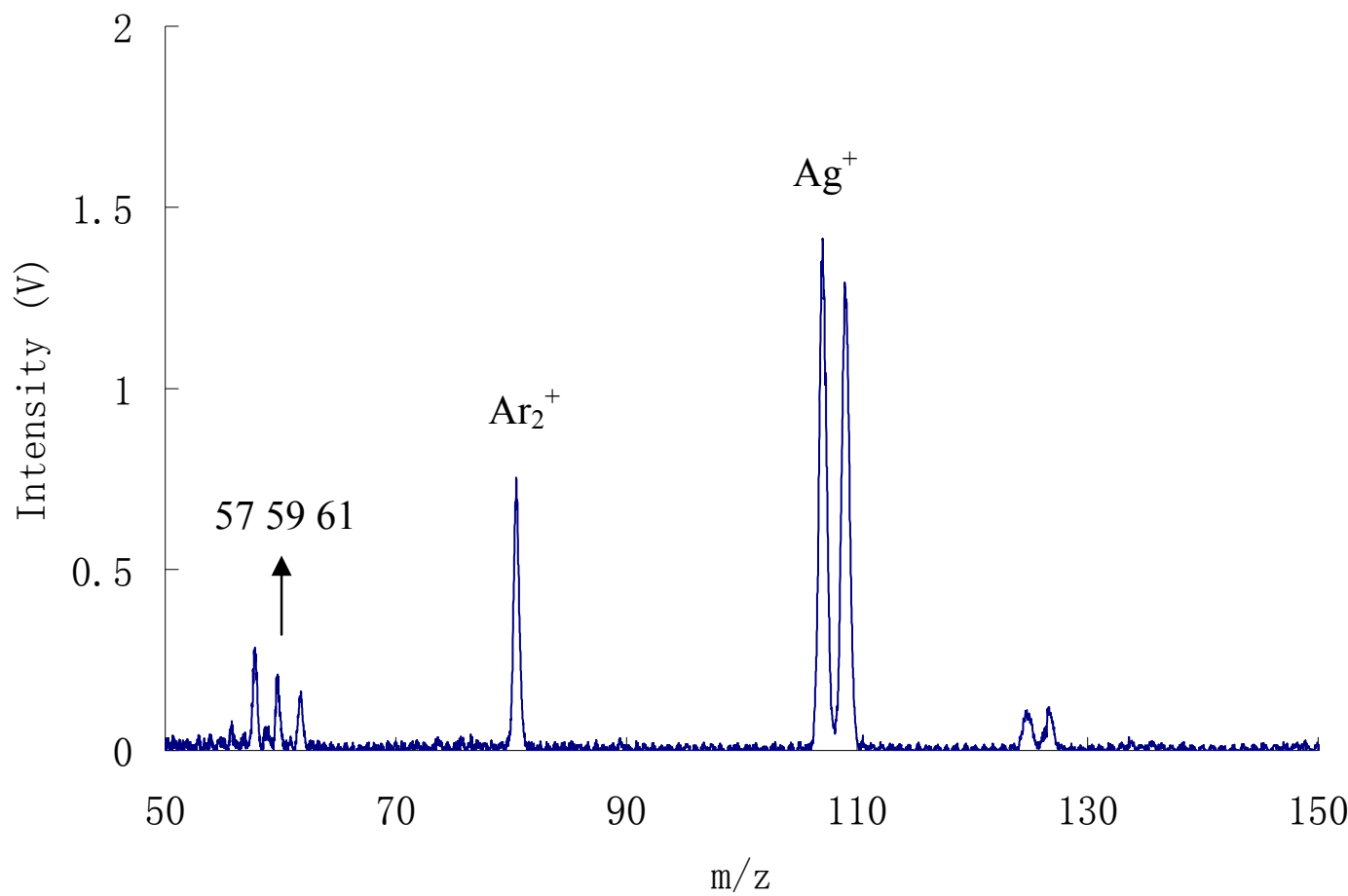

(b) Leucine

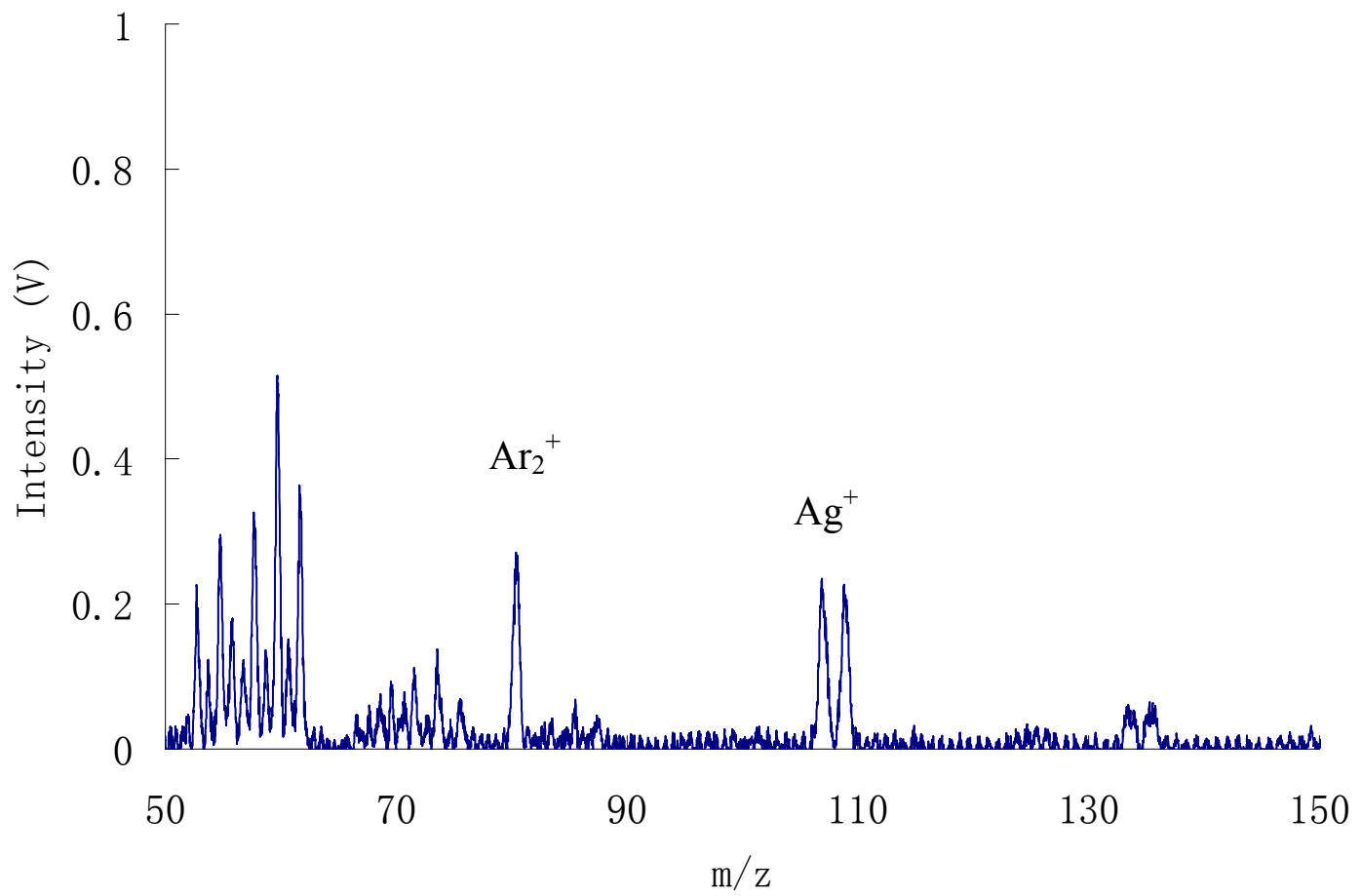


(c) Cysteine

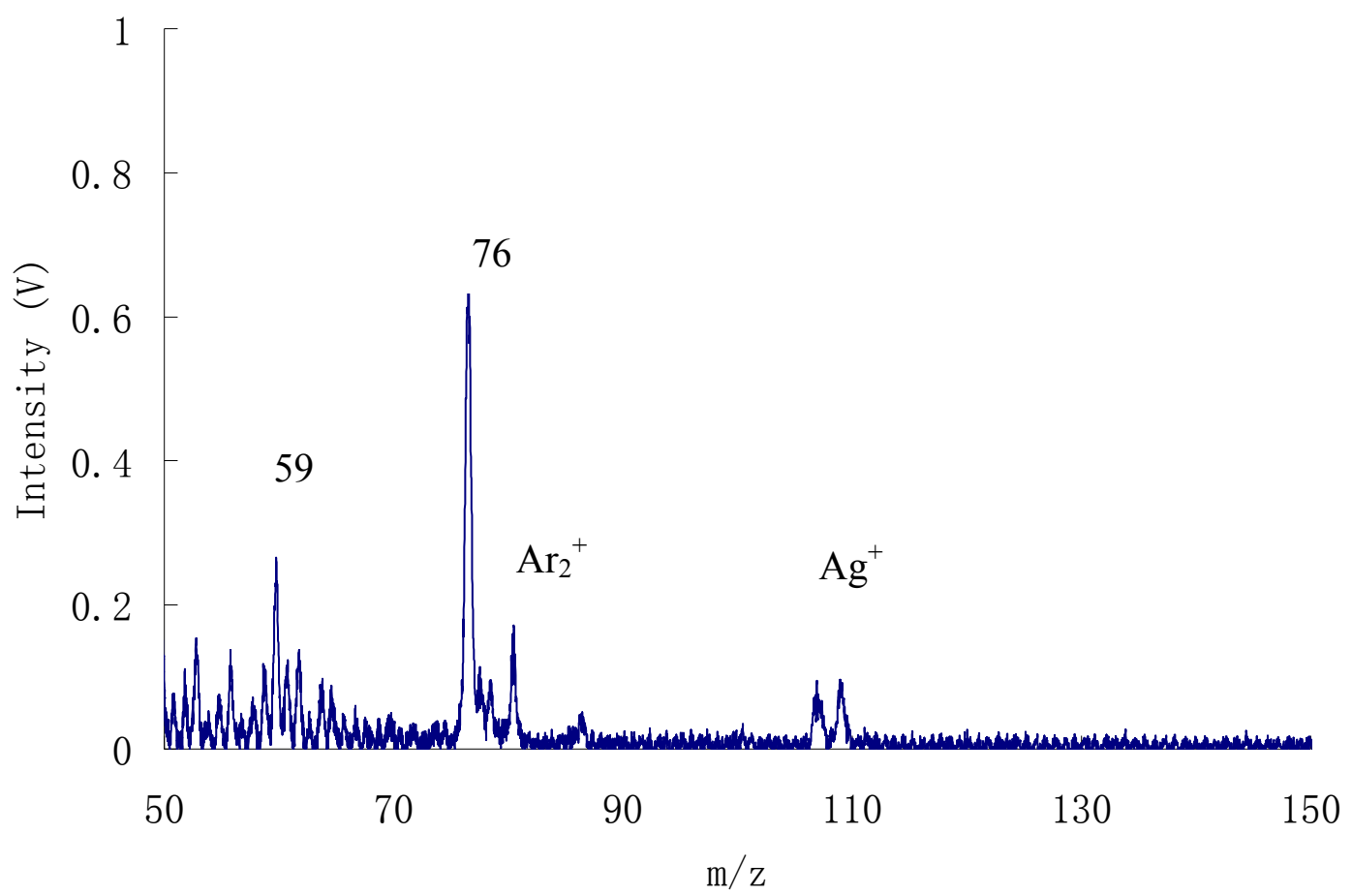

(d) GlyGlyHis

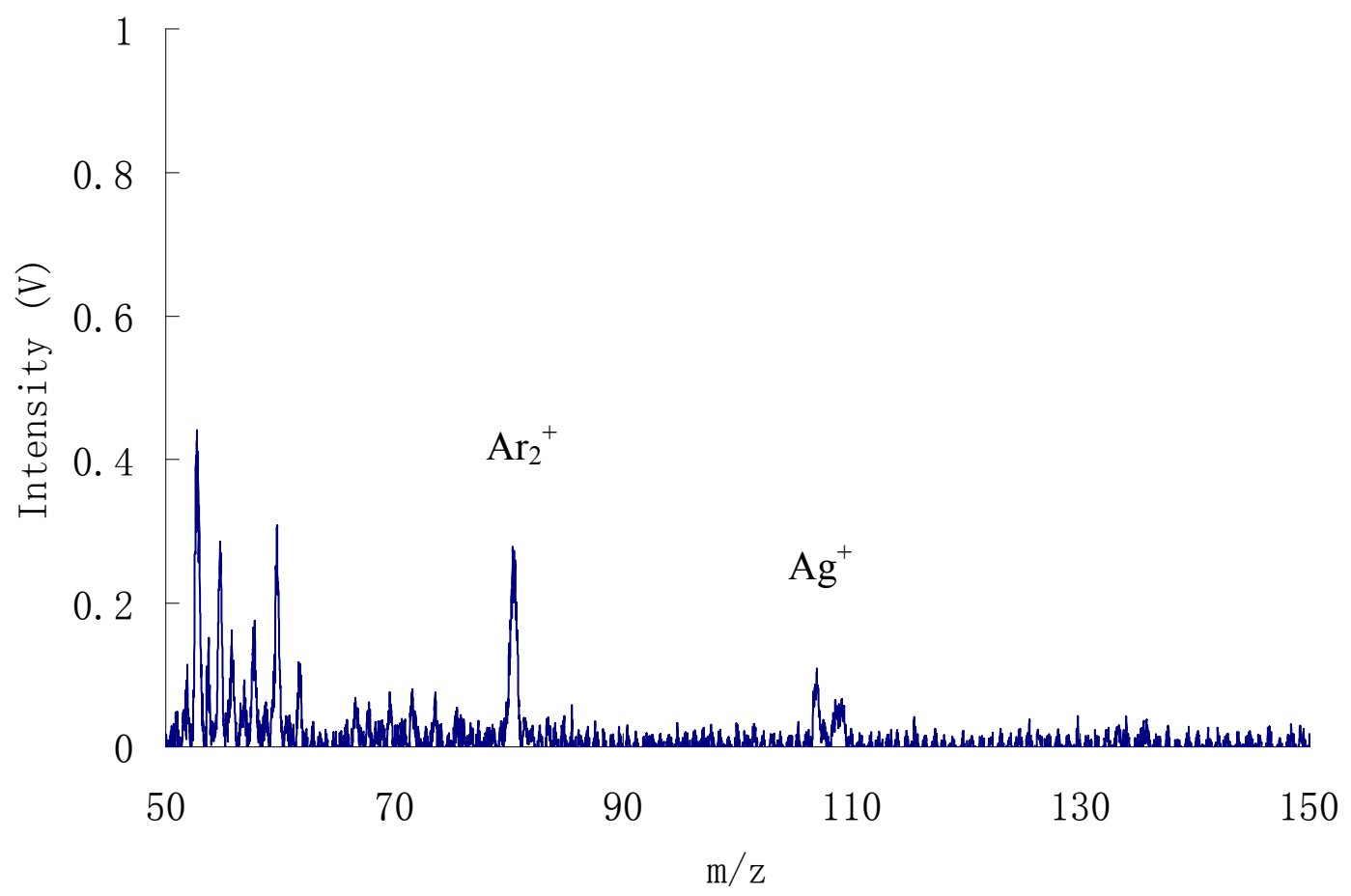

Figure 5.3 Mass spectra of (a) Ag, (b) Leucine, (c) Cysteine, and (d) GlyGlyHis samples during the afterpeak (0.2 ms after power-off). Discharge pressure 0.3 Torr, sampling distance $15 \mathrm{~mm}$, peak power $80 \mathrm{~W}$ with $100 \mathrm{~Hz}$ frequency and $10 \%$ duty cycle. 


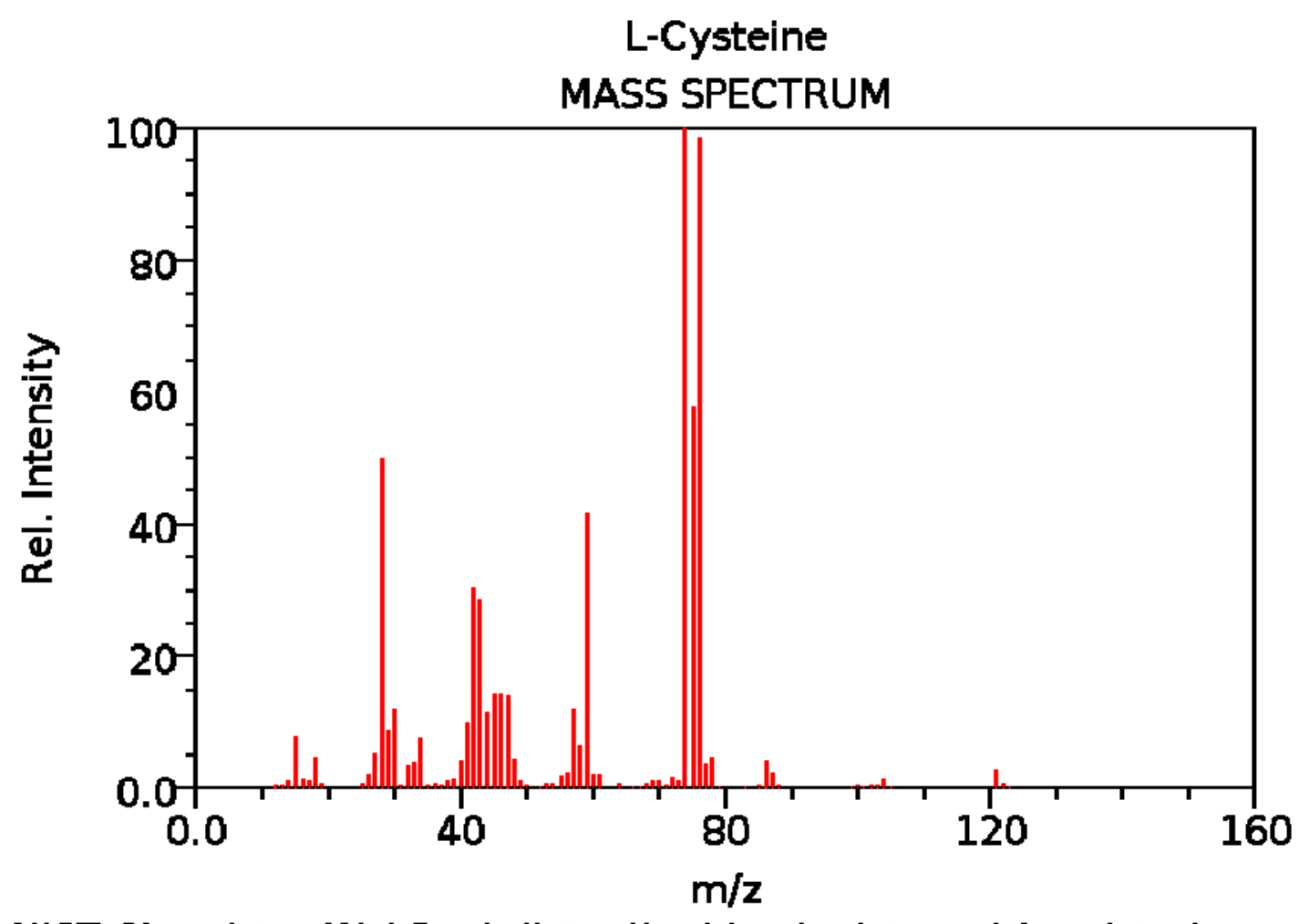

NIST Chemlstry WebBook (http://webbook.nlst.gov/chemlstry)

Figure 5.4 Standard EI mass spectrum of cysteine. ${ }^{21}$

\subsubsection{Sample and CsCl Solution Analysis}

Another thought was to explore the possibility of adduct formation with a strongly ionizing species as a means of detecting amino acids. At the same time, the possible formation of clusters would shift the characteristic ion masses out of the range where significant background noise was observed, between m/z 50 and 60 . Cesium chloride was added to the solution to promote clustering and adduct formation. Cesium has a very low first ionization energy $(3.89 \mathrm{eV})$ and should be readily ionized in the glow discharge making it available for adduct formation as well as consuming excess plasma energy (plasma quenching) that otherwise might go in to 
unwanted molecular dissociation.

Figure 5.5 shows mass spectra of different samples tested in this method. Cesium chloride $(\mathrm{CsCl})$ sample is tested as blank. Its mass spectrum shows $\mathrm{CsCl}^{+}$ions $(\mathrm{m} / \mathrm{z}$ $168,170)$ and strong $\mathrm{Cs}^{+}(\mathrm{m} / \mathrm{z}$ 133) signal. The mass spectrum of cysteine in $\mathrm{CsCl}$ solution is presented as an example of what was found with this approach. Clearly, neither larger cluster ions nor other characteristic ions were observed. Interestingly the previously observed $\mathrm{m} / \mathrm{z} 76$ signal from cysteine is also absent. This leads one to conclude that not only does the Cs not form adducts or lead to clustering, but it also shifts ionization away from the cysteine that was observed previously. The tripeptide/CsCl sample exhibits similar behavior. 
(a) $\mathrm{CsCl}$

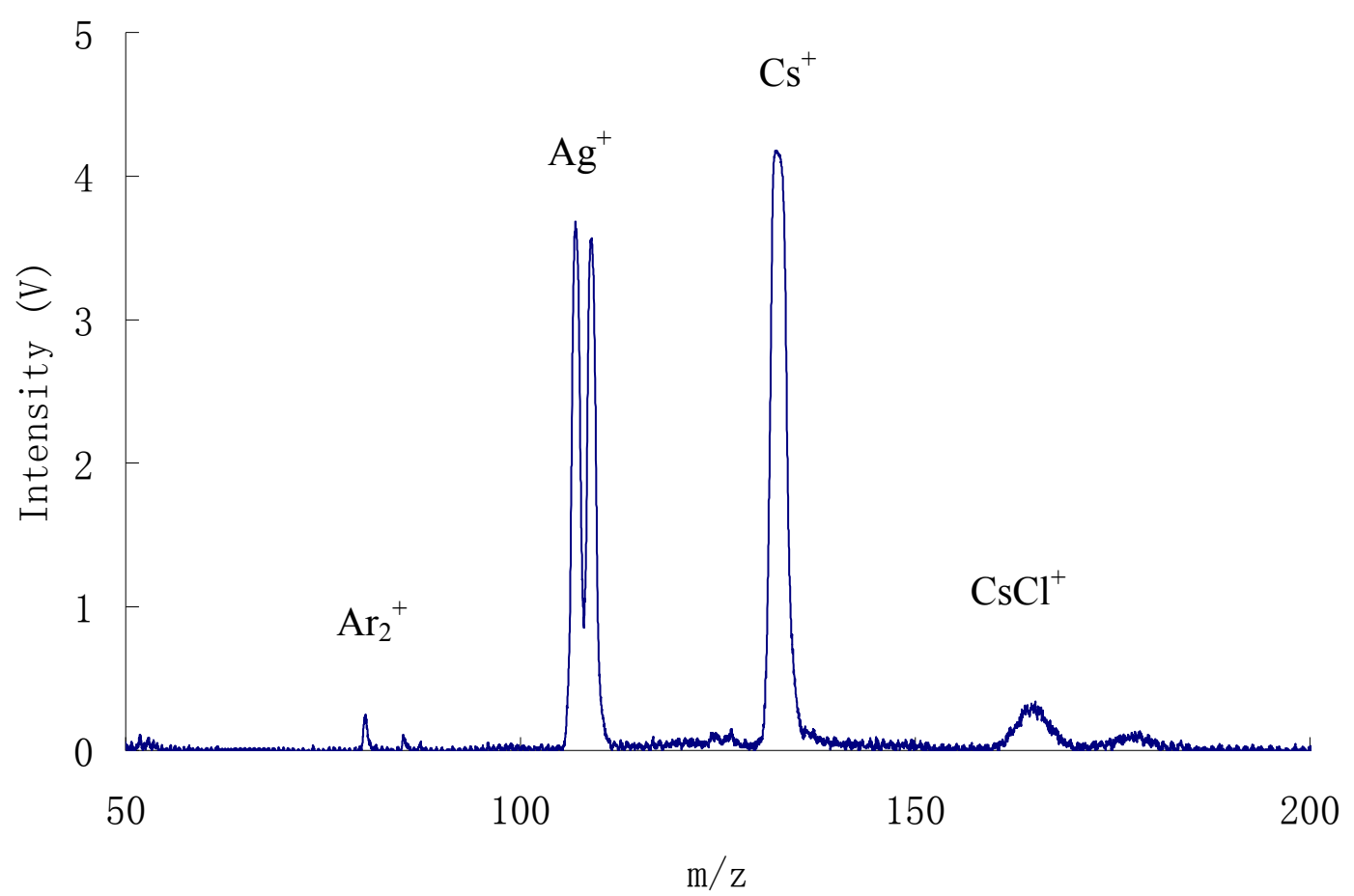

(b) $\mathrm{CsCl}+$ Cysteine

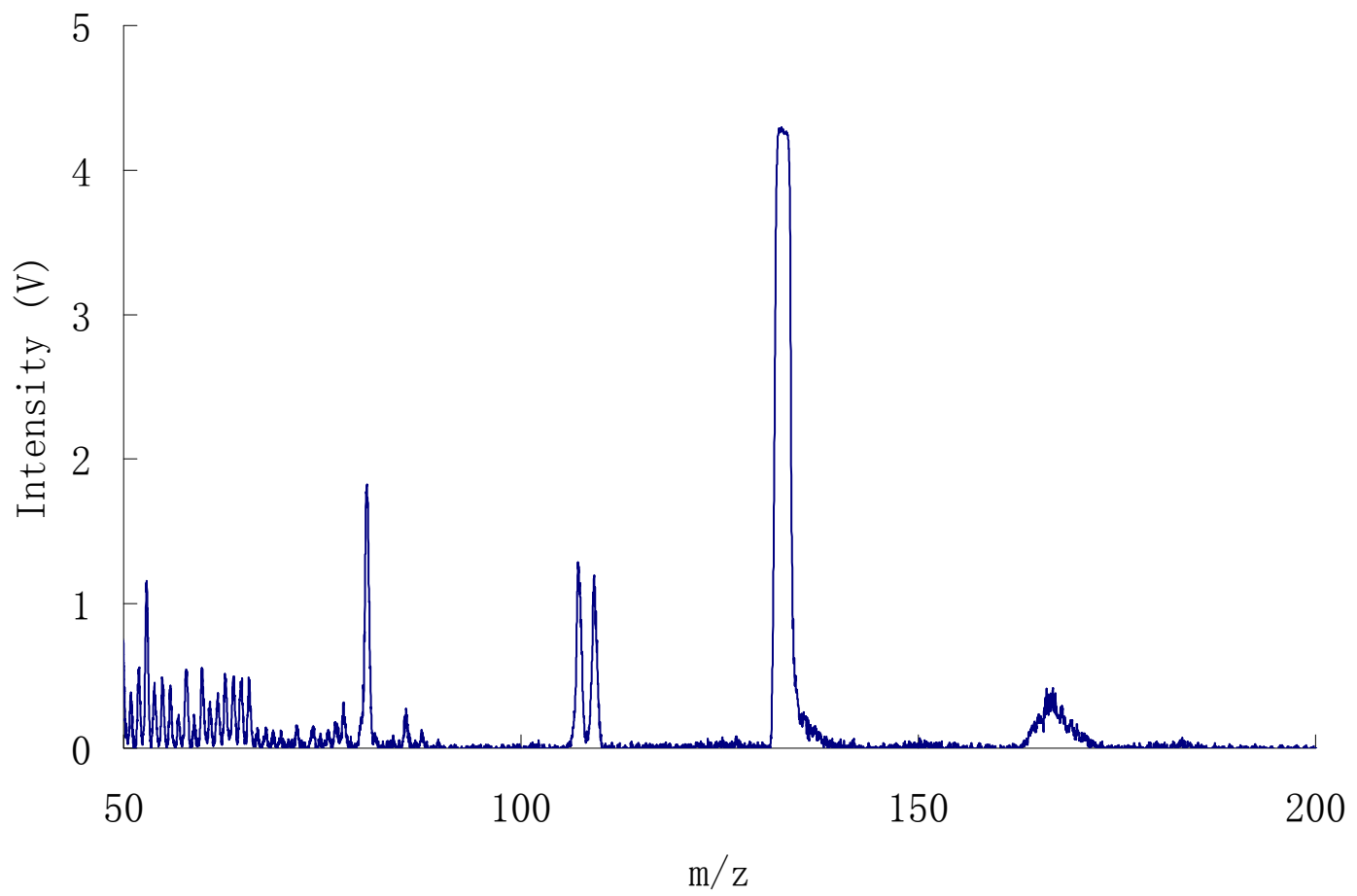


(c) $\mathrm{CsCl}+$ GlyGlyHis

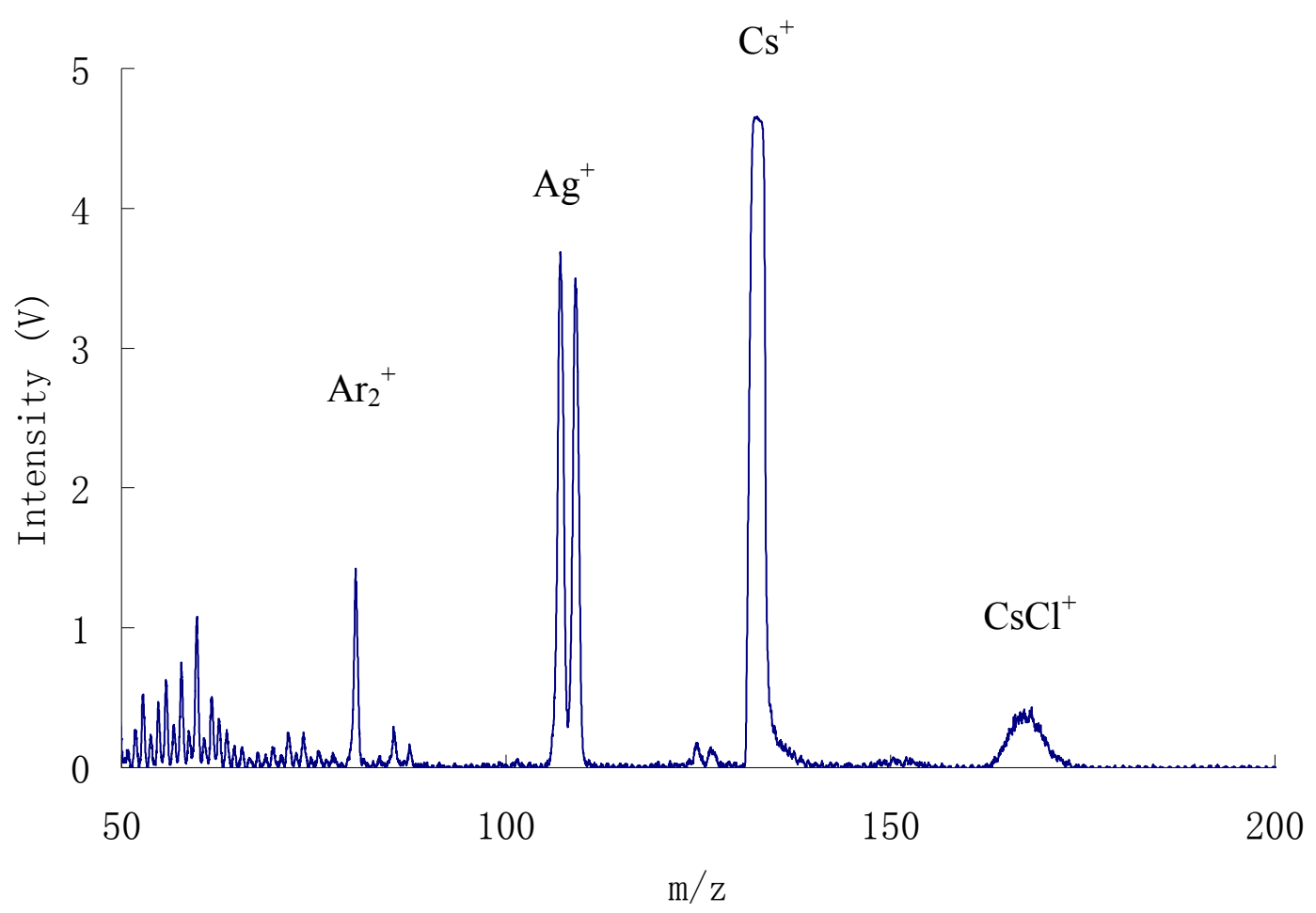

Figure 5.5 Mass spectra of (a) $\mathrm{CsCl}$, (b) $\mathrm{CsCl}+$ Cysteine, and (c) $\mathrm{CsCl}+$ GlyGlyHis samples during the afterpeak (0.2 ms after power-off). Discharge pressure 0.3 Torr, sampling distance $15 \mathrm{~mm}$, peak power $80 \mathrm{~W}$ with $100 \mathrm{~Hz}$ frequency and $10 \%$ duty cycle.

\subsection{Conclusion}

The direct analysis of bio-molecules, such as amino acids and peptide, by glow discharge mass spectrometry was explored. Three different sample preparation methods were employed to explore the role of solvents or additives. Glycerol was explored to mimic FAB and LSIMS experimental condition in the first method, but no molecular ion or characteristic ions were detected. In the second method, samples were dissolved in deionized water and a sample solution droplet was deposited on the 
sample disk. Among six amino acids and one tripeptide tested in this method, only cysteine showed a characteristic ion, $\mathrm{m} / \mathrm{z} 76$, arising from carboxylic acid loss. This ion was also observed in EI and SIMS spectra reported in the literature. In the last method, bio-molecules were dissolved in $10 \mathrm{mM}$ cesium chloride solution instead of deionized water, but the expected cesium related cluster ion was not detected in the spectra. There were no molecular or characteristic ions detected with that approach.

In general, it is concluded that this approach is not promising for peptide determinations. More study into the question of the apparently unique observation for $\mathrm{m} / \mathrm{z}$ from 76 from cysteine will be explored in the future. Cysteine-containing peptides will be explored to determine if they also exhibit this characteristic ion. 


\subsection{References}

1. R. Kenneth Marcus, Glow Discharge Spectroscopies, Plenum Press, New York, 1993

2. A. P. Mykytiuk, P. Semeniuk, and S. Berman, Analysis of High Purity Metals and Semiconductor Materials by Glow Discharge Mass Spectrometry. Spectrochim. Acta Rev. 1990, 13, 1

3. Applicarion Note, GDMS-AN-002-01, Charles Evans \& Associates, Redwood City, Calif., 1988

4. S. A. McLuckey, G. L. Glish, and K. G. Asano, The Coupling of an Atmospheric Sampling Ion Source with an Ion Trap Mass SpectrometeR. Anal. Chim. Acta., $1989,225,25$

5. Cris L. Lewis, Mathew A. moser, Don E. Dale Jr., Wei Hang, Christian Hassell, Fred L. King, and Vahid Majidi, Time-Gated Pulsed Glow Discharge: Realtime Chemical Speciation at the Elemental, Structural, and Molecular Level for Gas Chromatography Time-of-Flight Mass Spectrometry. Anal. Chem. 2003, 75, $1983-96$

6. W. A. Mattson, B. L. Bentz, and W. W. Harrison, Coaxial Cathode Ion Source for Solids Mass Spectrometry. Anal. Chem, 1976, 48, 489

7. N. Jakubowski, D. Stuewer, and G. Toelg, Microchemical Determination of Platinum and Iridium by Glow Discharge Mass Spectrometry. Spectrochim. Acta Part B: Atomic Spectroscopy. 1991, 46, 155

8. Quarles, C. Derrick, Jr.; Niemann, Scott; Marcus, R. Kenneth, Conversion of a 
Commercial gas Chromatography-Mass Spectrometer to a Liquid

Chromatography-Particle Beam/Glow Discharge Mass Spectrometer. Journal of Analytical Atomic Spectrometry. 2010, 25 (11), 1780-1786

9. Castro, Joaudimir; Krishna, M. V. Balarama; Choiniere, John R.; Marcus, R. Kenneth, Analysis of caffeic acid derivatives in echinacea extracts by liquid chromatography particle beam mass spectrometry (LC-PB/MS) employing electron impact and glow discharge ionization sources. Analytical and Bioanalytical Chemistry. 2010, 397(3), 1259-1271

10. Venzie, Jacob L.; Castro, Joaudimir; et al., and Marcus, R. Kenneth. Electron-Impact and Glow-Discharge Ionization LC-MS Analysis of Green Tea Tincture. Analytical and Bioanalytical Chemistry. 2007, 387(1), 321-333

11. Brewer, Tim M.; Castro, Joaudimir; Marcus, R. Kenneth, Particle Beam Sample Introduction into Glow Discharge Plasmas for Speciation Analysis. Spectrochimica Acta, Part B: Atomic Spectroscopy. 2006, 61B(2), 134-149

12. Venzie, Jacob L.; Davis, W. Clay; Marcus, R. Kenneth, Organic and Inorganic Arsenic Speciation Through Ion Exchange Chromatography with Particle Beam-Glow Discharge Mass Spectrometry Detection. Journal of Analytical Atomic Spectrometry. 2004, 19(10), 1309-1314

13. Gibeau, T. E.; Marcus, R. K., Glow Discharge Ionization Source for Liquid Chromatography/Particle Beam Mass Spectrometry. Analytical Chemistry. 2000, 72(16), 3833-3840 
14. A. Benninghoven, D. Jaspers, and W. Sichtermann, Advances in Mass Spectrometry, 1978, 7B, 1433-36

15. W. Aberth, K. M. Straub, and A. L. Burlingame, Secondary Ion Mass Spectrometry with Cesium Ion Primary Beam and Liquid Target Matrix for Analysis of Bioorganic Compounds. Anal Chem. 1982, 54, 2029-34

16. http://biotech.matcmadison.edu/resources/proteins/labManual/chapter_2.htm

17. Jennifer N. Robertson-Honecker, Na Zhang, Alexandria Pavkovichab and Fred L. King, Direct Chromium Speciation in Solid State Materials - a GDMS Approach, J. Anal. At. Spectrom., 2008, 23, 1508-1517.

18. Na Zhang and Fred L. King, Direct Manganese (Mn) Speciation in Solid State Materials by Pulsed Glow Discharge Time-of-Flight Mass Spectrometry, J. Anal. At. Spectrom. 2009, 24(11), 1489-1497.

19. Guodong Gu, Megan DeJesus, and Fred L. King, Direct FexOy Speciation in Solid State Materials by Pulsed Millisecond Radio Frequency Glow Discharge Time-of-Flight Mass Spectrometry, J. Anal. At. Spectrom., 2011, 26, 816-21.

20. M. Barber, R.S. Bardoli, R.D. Sedgwick, and A.H. Tyler, Fast Atom Bombardment of Solids (F.A.B.): a New Ion Source for Mass Spectrometry. J. Chem. Soc., Chem. Commu., 1981, 325

21. http://webbook.nist.gov/chemistry 